SANDIA REPORT

SAND95-2338 - UC-814

Unlimited Release

Printed August 1996
RECFIVED

SEP 261996

OSTI

Yucca Mountain Site Characterization Project

\title{
Scaling of Material Properties for Yucca Mountain: Literature Review and Numerical Experiments on Saturated Hydraulic Conductivity
}

\author{
S. A. McKenna, C. A. Rautman
}

Prepared by

Sandia National Laboratories

Albuquerque, New Mexico 87185 and Livermore, California 94550

for the United States Department of Energy

under Contract DE-AC04-94ALE5000

Approved for public release; distribution is unlimited.
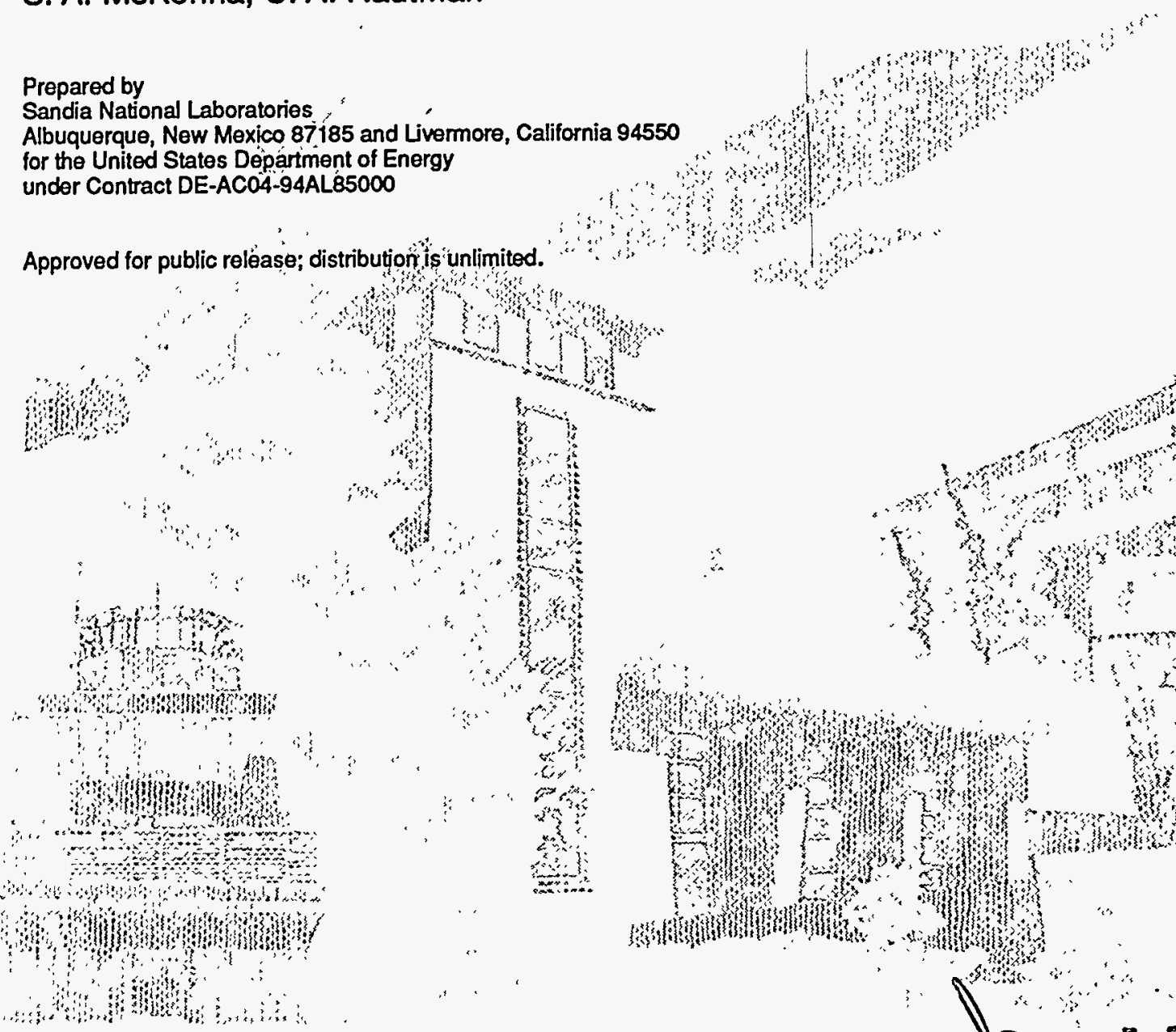

SF29000(8-81)

DISTRBUTION OF THIS DOCURENT IS URLLITED

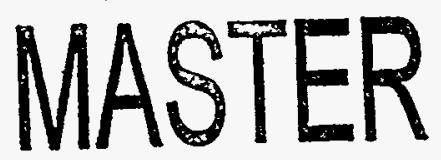


"Prepared by Yucca Mountain Site Characterization Project (YMSCP) participants as part of the Civilian Radioactive Waste Management Program (CRWM). The YMSCP is managed by the Yucca Mountain Project Office of the U.S. Department of Energy, DOE Field Office, Nevada (DOE/NV). YMSCP work is sponsored by the Office of Geologic Repositories (OGR) of the DOE Office of Civilian Radioactive Waste Management (OCRWM)."

Issued by Sandia National Laboratories, operated for the United States Department of Energy by Sandia Corporation.

NOTICE: This report was prepared as an account of work sponsored by an agency of the United States Government. Neither the United States Government nor any agency thereof, nor any of their employees, nor any of their contractors, subcontractors, or their employees, makes any warranty, express or implied, or assumes any legal liability or responsibility for the accuracy, completeness, or usefulness of any information, apparatus, product, or process disclosed, or represents that its use would not infringe privately owned rights. Reference herein to any specific commercial product, process, or service by trade name, trademark, manufacturer, or otherwise, does not necessarily constitute or imply its endorsement, recommendation, or favoring by the United States Government, any agency thereof or any of their contractors or subcontractors. The views and opinions expressed herein do not necessarily state or reflect those of the United States Government, any agency thereof or any of their contractors.

Printed in the United States of America. This report has been reproduced directly from the best available copy.

Available to DOE and DOE contractors from

Office of Scientific and Technical Information

PO Box 62

Oak Ridge, TN 37831

Prices available from (615) 576-8401, FTS 626-8401

Available to the public from

National Technical Information Service

US Department of Commerce

5285 Port Royal Rd

Springfield, VA 22161

NTIS price codes

Printed copy: A06

Microfiche copy: A01 


\section{DISCLAIMER}

Portions of this document may be illegible in electronic image products. Images are produced from the best available original document. 
SAND95-2338

Unlimited Release

Printed August 1996
Distribution Category

UC-814

\title{
Scaling of Material Properties for Yucca Mountain: Literature Review and Numerical Experiments on Saturated Hydraulic Conductivity
}

\author{
Sean A. McKenna and Christopher A. Rautman \\ Geohydrology Department \\ Sandia National Laboratories
}

\section{Abstract}

A review of pertinent literature reveals techniques which may be practical for upscaling saturated hydraulic conductivity at Yucca Mountain: geometric mean, spatial averaging, inverse numerical modeling, renormalization, and a perturbation technique. Isotropic realizations of log hydraulic conductivity exhibiting various spatial correlation lengths are scaled from the point values to five discrete scales through these techniques. For the variances in $\log _{10}$ saturated hydraulic conductivity examined here, geometric mean, numerical inverse and renormalization adequately reproduce point scale fluxes across the modeled domains. Fastest particle velocities and dispersion measured on the point scale are not reproduced by the upscaled fields. Addtional numerical experiments examine the utility of power law averaging on a geostatistical realization of a crosssection similar to the cross-sections that will be used in the 1995 groundwater travel time calculations. A literature review on scaling techniques for thermal and mechanical properties is included.

This work was supported by the U.S. Department of Energy under contract DE-AC0494AL85000, WBS Element 1.2.3.2.2.2.2, Work Agreement 0015, QAGR 1.2.3.2.2.2.2 RO. Data for borhole UZ-16 are unqualified under DTN GS940508312231.006. The work in this report was done under qualified procedures. 


\section{Table of Contents}

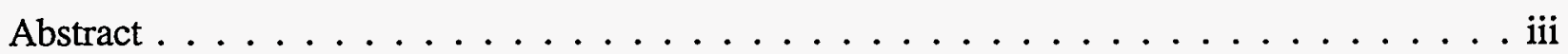

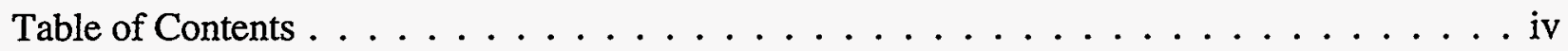

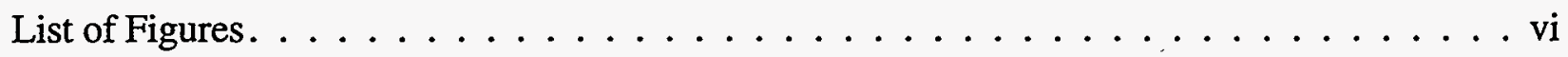

List of Tables .......................... . . . . . . .

List of Symbols . . . . . . . . . . . . . . . . . . . . x

Introduction ............................

Application of Scaling Methods to Yucca Mountain . . . . . . . . . . . . . . 2

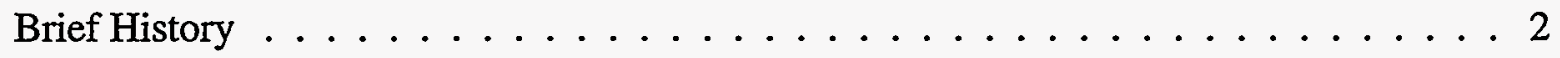

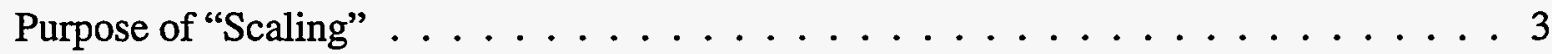

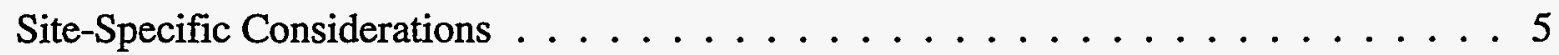

Requirements of Scaling Algorithms for Use at Yucca Mountain . . . . . . . . . . 5

Description of Scaling Algorithms: A Literature Review . . . . . . . . . . . . . . 6

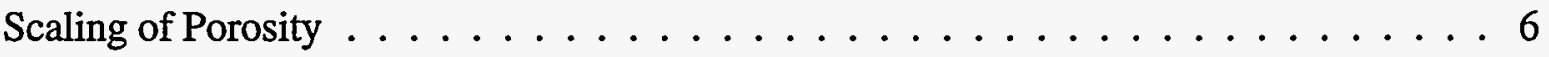

Scaling of Saturated Hydraulic Conductivity . . . . . . . . . . . 8

Analytical Expressions . . . . . . . . . . . . . . . . . . . . . . . . . . . . . . . . .

Geometric Mean. . . . . . . . . . . . . . . . . 9

Harmonic Mean . . . . . . . . . . . . . . . . . . 10

Arithmetic Mean . . . . . . . . . . . . . . . . . 10

Bounding the Effective Conductivity Value . . . . . . . . . . 11

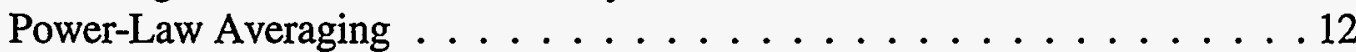

Spatial Averaging . . . . . . . . . . . . . . . . 14

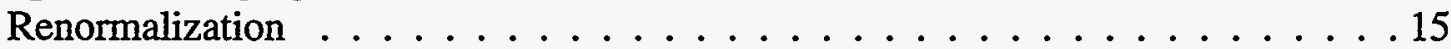

Numerical-Inverse Modeling . . . . . . . . . . . . . . . . . . . . . . . . . . . . . . . . . .

Effective-Medium Theory . . . . . . . . . . . . . 17

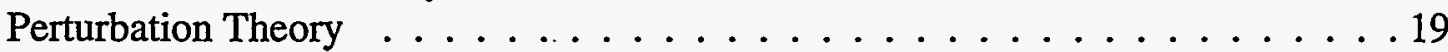

Scaling of Unsaturated Hydrologic Properties . . . . . . . . . . . . . . . . 21

Evaluation of Proposed Scaling Algorithms: Numerical Experiments . . . . . . . . . . . . 22

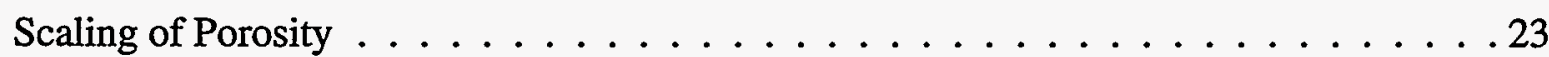

Experiment Summary and Results . . . . . . . . . . . . . 23

Scaling of Saturated Hydraulic Conductivity . . . . . . . . . . . . . . . . . . . 24

Empirical Approach and Definition of Performance Measures for Flow and Transport 26

Generation of Point-Scale, Isotropic, Spatially Correlated Conductivity Fields . . . 27

Generation of Point-Scale, Anisotropic, Conductivity Field . . . . . . . . . . . 27

Scaling of Isotropic Conductivity Fields . . . . . . . . . . . . . . . . 33

Scaling of the Anisotropic, "Yucca Mountain" Conductivity Field . . . . . . . . . 34

Flow-Model Set Up . . . . . . . . . . . . . . . . . . . 36

Flow and Transport Results for Point-Scale, Isotropic Conductivity Grids . . . . . . . 38

Flow-and-Transport Results for Scaled, Isotropic Conductivity Grids . . . . . . . . . . 44 
Effect of Upscaling on Variance . . . . . . . . . . . . . . . 46

Effect of Variability and Correlation Length on Upscaling of Isotropic Fields . . .47

Flow Properties . . . . . . . . . . . . . . . . . . 52

Transport properties . . . . . . . . . . . . . . . 52

Flow-and-Transport Results for Anisotropic Conductivity Profile. . . . . . . . 60

Flow Properties . . . . . . . . . . . . . . . . . . 61

Transport Properties . . . . . . . . . . . . . 63

Discussion. . . . . . . . . . . . . . . . . . 67

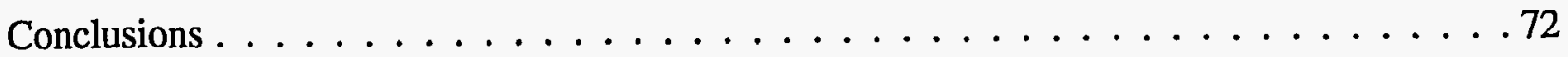

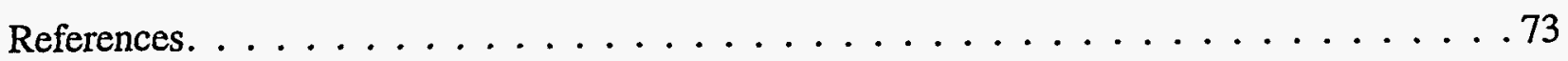

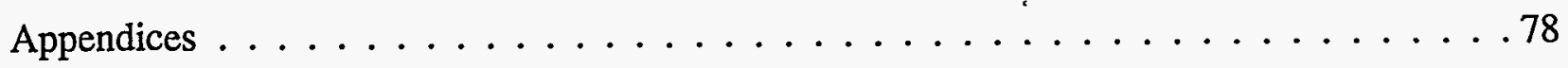




\section{List of Figures}

Figure 1. Schematic representation of flow (A) perpendicular and (B) parallel to layering in a two-dimensional domain. Notation corresponds to that used in the equations in the

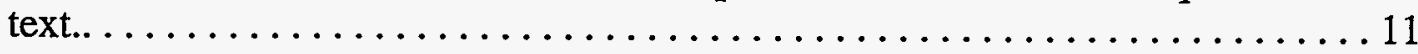

Figure 2. Conceptual representation of grids used in the numerical-inverse technique for upscaling. (A) Five-by-five grid of cells to be upscaled to a single block of size $5 \times 5$. (B) The same $5 \times 5$ grid embedded in a "skin" of flow elements. Bold lines parallel to the flow direction are no flow boundaries and the dashed lines perpendicular to flow are constant head boundaries. . . . . . . . . . . . . . . . . 16

Figure 3. Conceptual representation of upscaling through effective-medium theory. A discretized domain of internally homogeneous blocks of hydraulic conductivity is replaced by a single isolated inclusion, with hydraulic conductivity equal to $\mathrm{K} 1$, embedded in an infinite domain with hydraulic conductivity equal to $\mathrm{KO}$. . . . . 17

Figure 4. Grey-scale coded, 256-by-256, point-scale fields of log hydraulic conductivity: (top) isotropic spatial correlation of $10 \mathrm{~cm}$; (bottom) $20 \mathrm{~cm}$. Maximum value is white, minimum value is black. . . . . . . . . . . . . . . . . . 28

Figure 5. Grey-scale coded, 256-by-256, point-scale fields of log hydraulic conductivity: (top) isotropic spatial correlation of $40 \mathrm{~cm}$; (bottom) $80 \mathrm{~cm}$. Maximum value is white, minimum value is black . . . . . . . . . . . . . . . . . . . . . 29

Figure 6. Grey-scale coded, 256-by-256, point-scale fields of log hydraulic conductivity: (top) isotropic spatial correlation of $160 \mathrm{~cm}$; (bottom) $320 \mathrm{~cm}$. Maximum value is white, minimum value is black. ......................... 30

Figure 7. Grey-scale coded, 256-by-256, point-scale field of log hydraulic conductivity with isotropic spatial correlation of $640 \mathrm{~cm}$. Maximum value is white, minimum value is

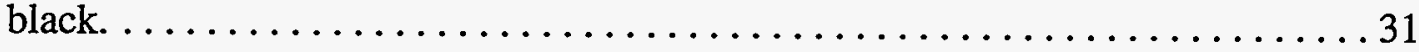

Figure 8. Simulated west-to-east, cross-sectional material-property model of Yucca Mountain for saturated hydraulic conductivity. Model has been created through a linear coregionalization of conductivity with porosity (Journel and Huijbregts, 1978). Values range from -12 (black) to -6 (white) $\mathrm{m} / \mathrm{sec}$ in $\log 10$ space... . . . . . . 32

Figure 9. Irregular grid for the cross section shown in figure 8 that globally minimizes withingrid-cell material-property heterogeneity. The "quasi-homogenized" grid cells form calculational elements for the flow-and-transport model. . . . . . . . . . 35

Figure 10. Example of 100 particle tracks through a heterogeneous point-scale log hydraulic conductivity field. . . . . . . . . . . . . . . . . . . 38

Figure 11. Box plots illustrating the distributions of arrival times for the seven point-scale realizations with progressively increasing ranges of spatial correlation: (a) standard deviation of $\log$ hydraulic conductivity equal to 0.5 ; (b) standard deviation of $\log$ hydraulic conductivity equal to 1.0. The thick dashed lines represent the mean, the thin dashed lines represent the median, and the boxes define the 10th, 25th, 75th and 90 th percentiles of the distributions. . . . . . . . . . . . . . 39

Figure 12. Percent of values in point-scale simulations that are within one standard deviation of 
the mean. The dashed line represents the theoretical value for a Gaussian distribution. ................................. 45

Figure 13. Conceptual representation of the five degrees of scaling examined by numerical experiments. The degree of scaling can be described as the number of calculational elements discretizing each side of the domain (e.g., 16x16) or by the number of pointscale values that constitute each upscaled element (256 into 1).. . . . . . . 46

Figure 14. A point-scale field conductivity field with a range of $160 \mathrm{~cm}$ scaled to the five progressive levels of scaling using the geometric mean as the scaling algorithm. . 47

Figure 15. Graphs showing the standard deviation of $\log$ hydraulic conductivity as a function of the degree of upscaling: (A) $\lambda=10 ;(B) ; \lambda=20 \ldots \ldots \ldots \ldots \ldots \ldots \ldots \ldots$

Figure 16. Graphs showing the standard deviation of $\log$ hydraulic conductivity as a function of the degree of upscaling: (A) $\lambda=40 ;(B) ; \lambda=80 \ldots \ldots \ldots \ldots \ldots \ldots \ldots$

Figure 17. Graphs showing the standard deviation of log hydraulic conductivity as a function of the degree of upscaling: (A) $\lambda=160 ;(B) ; \lambda=320 \ldots \ldots \ldots \ldots \ldots \ldots$

Figure 18. Graph showing percent error in the computed hydraulic conductivity of the $2560-\mathrm{cm}$ by $2560-\mathrm{cm}$ flow model as a function of grid-element length to correlation length ratio $\left(\mathrm{L}_{\mathrm{e}} / \mathrm{l}\right)$. Standard deviation of underlying point-scale conductivity simulations

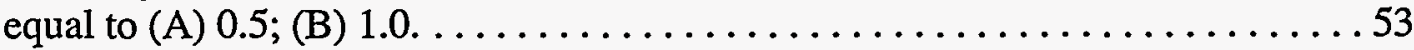

Figure 19. Graph showing percent error in mean particle velocity through the 2560 -cm by 2560 $\mathrm{cm}$ flow model as a function of grid-element length to correlation length ratio $\left(\mathrm{L}_{\mathrm{e}} /\right)$. Standard deviation of underlying point-scale conductivity simulations equal to (a)

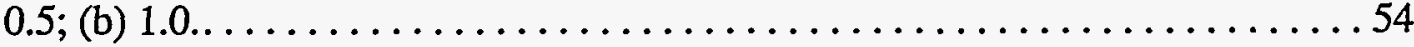

Figure 20. Graph showing percent error in fastest particle velocity through the $2560-\mathrm{cm}$ by $2560-\mathrm{cm}$ flow model as a function of grid-element length to correlation length ratio $\left(\mathrm{L}_{\mathrm{e}} / \mathrm{l}\right)$. Standard deviation of underlying point-scale conductivity simulations equal to

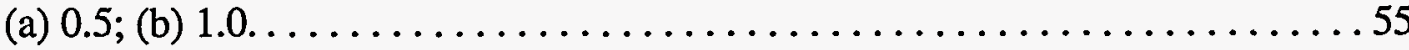

Figure 21. Graph showing percent error in longitudinal dispersivity through the 2560 -cm by $2560-\mathrm{cm}$ flow model as a function of grid-element length to correlation length ratio $\left(\mathrm{L}_{\mathrm{e}} / \mathrm{l}\right)$. Standard deviation of underlying point-scale conductivity simulations equal to

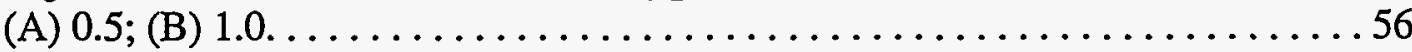

Figure 22. Graph showing percent error in longitudinal dispersion coefficient as a function of grid-element length to correlation length ratio $\left(L_{e} / 1\right)$. Standard deviation of underlying point-scale conductivity simulations equal to (A) 0.5 ; (B) 1.0 . . . 57

Figure 23. Graph showing dispersivity (al )values that will reproduce the amount of point-scale dispersion as a function of grid-element length to correlation length ratio $\left(L_{e} / l\right)$. Standard deviation of underlying point-scale conductivity simulations equal to

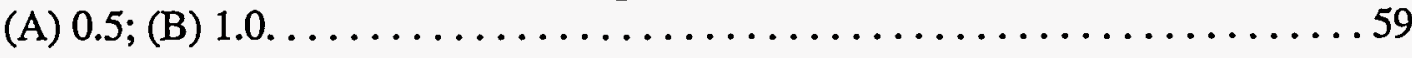

Figure 24. Graph showing percent error reproducing effective hydraulic conductivity of the simulated cross-sectional model of Yucca Mountain as a function of the power-law

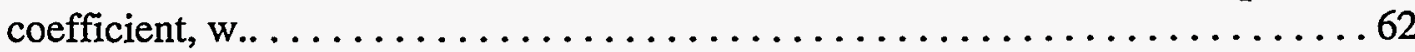


Figure 25. Graph showing percent error reproducing average travel time through the simulated cross-sectional model of Yucca Mountain as a function of the power-law coefficient,

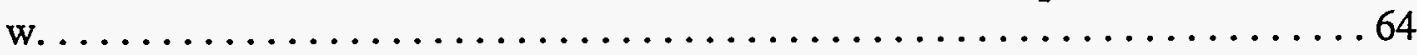

Figure 26. Graph showing percent error reproducing fastest travel time through the simulated cross-sectional model of Yucca Mountain as a function of the power-law coefficient,

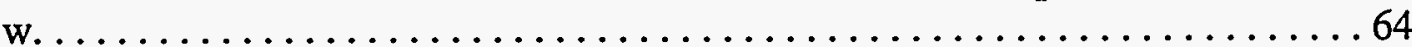

Figure 27. Graph showing percent error reproducing dispersivity through the simulated crosssectional model of Yucca Mountain as a function of the power-law coefficient, w. . 65

Figure 28. Graph showing percent error reproducing the longitudinal dispersion coefficient through the simulated cross-sectional model of Yucca Mountain as a function of the power-law coefficient, w. . . . . . . . . . . . . . . . . . . . . . . 66

Figure 29. Comparison of hydraulic conductivity values upscaled by the spatial average and the geometric mean (top) for the field with a range of 20 and upscaled to elements with a length of $40 \times 40$. The bottom graph compares the geometric mean with the numerical inverse solution. Relative to other techniques, such as the numerical inverse solution, spatial averaging produces a larger variance and lower values.. . 69 


\section{List of Tables}

Table 1: Comparison of standard deviations predicted by the volume-variance relationship of Clark (1979) and the actual standard deviation of larger-scale blocks computed by arithmetic averaging . . . . . . . . . . . . . . . 24

Table 2: Scaling Techniques Selected for Numerical Experimentation . . . . . . . . . 25

Table 3: Results of Flow-and-Transport Calculations for Original Point-Scale Grids Without Up-

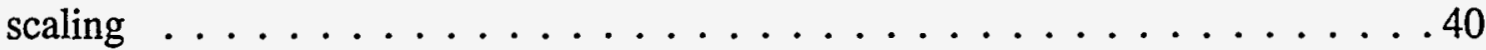

Table 4: Comparison of dispersivities calculated for the point-scale hydraulic conductivity fields and dispersivities predicted by the expression in Gelhar and Axness (1983). . . . . 41

Table 5: Matrix showing equivalent degrees of scaling as indicated by equal ratios of flow-element length to spatial-correlation length $\left(\mathrm{L}_{e} / 1\right) \ldots \ldots \ldots \ldots \ldots \ldots \ldots$

Table 6: Flow-and-Transport Results Using Power-Law Averaging to Create Upscaled FlowModel Hydraulic Conductivity Values from Simulated Point-Scale Conductivities . 61 


\section{List of Symbols}

\begin{tabular}{clcll}
\hline Symbol & \multicolumn{1}{c}{ Description } & Symbol & \multicolumn{1}{c}{ Description } \\
\hline$\Phi$ & Porosity & $N$ & Number of samples \\
$K_{i}$ & Saturated hydraulic conductivity measurement & $\mu$ & Mean \\
$K_{e f f}$ & Effective hydraulic conductivity & $\sigma^{2}$ & Variance \\
$K_{G}$ & Geometric mean hydraulic conductivity & $\gamma$ & Variogram \\
$K_{j w}$ & $j^{\text {th }}$ Wisorized geometric mean hydraulic conduct. & $w$ & Exponent for power-law average \\
$K_{H}$ & Harmonic mean hydraulicconductivity & exp & Exponentiation operator \\
$K_{A}$ & Arithmetic mean hydraulic conductivity & $Q$ & Darcy flux \\
$K_{w}$ & Power-law average hydraulic conductivity & $A$ & Area \\
$K_{s s}$ & Hydraulic conductivity of sandstone & $V_{s s}$ & Volume fraction of sandstone \\
$K_{s h}$ & Hydraulic conductivity of shale & $V_{s h}$ & Volume fraction of shale \\
$K^{+}$ & Maximum hydraulic conductivity & $P e$ & Peclet number \\
$K$ & Minimum hydraulic conductivity & $L_{e}$ & Length of flow-model element \\
$K_{x x}$ & Hydraulic conductivity in prinicpal direction, X & $L_{f}$ & Macroscopic flow length \\
$K_{s y}$ & Hydraulic conductivity in prinicpal direction, $\mathrm{Y}$ & $L_{D}$ & Length of domain \\
$K_{z z}$ & Hydraulic conductivity in prinicpal direction, $Z$ & $\lambda$ & Spatial correlation length \\
$D_{l}$ & Longitudinal dispersivity coefficient & $V_{x}$ & Average particle velocity \\
$\alpha_{\lambda}$ & Dispersivity & $t$ & Travel time \\
\hline
\end{tabular}




\section{Introduction}

Volcanic rocks within the unsaturated zone at Yucca Mountain, Nevada are being considered as the potential location of a high-level nuclear-waste repository. These rocks, dominantly silicic tuffs, are being investigated to characterize the spatial variability of rock properties and to identify lithologic controls on these rock properties. The final result of these studies will be threedimensional material-property models of the Yucca Mountain site. These three-dimensional material-property models will be input to hydrologic-flow models and other numerical, physical-process modeling for use in design calculations, and in performance assessment studies.

An important part of creating three-dimensional material-property models is to determine how rock properties measured on one scale should be represented in numerical performanceassessment models that typically use computational grid blocks many orders of magnitude larger than the original samples. These properties are often measured on a core segment a few centimeters in diameter and a few centimeters long. For some material properties, a simple arithmetic average of actual or modeled core-scale property values provides a reasonable approximation of the effective value of the larger numerical model element. In other cases, notably for saturated hydraulic conductivity, such simple averages of core-scale data do not generally provide an accurate estimate of the property at the larger scale, and in fact the use of arithmetic averaging may distort the physical process being modeled and lead to inaccurate conclusions.

This report contains a summary of work conducted to determine valid scaling techniques for saturated hydraulic conductivity for use by the Yucca Mountain Project. The report presents the results of a literature review for nine published approaches to scaling hydrologic properties such as hydraulic conductivity, and it describes a series of numerical experiments implementing the scaling of saturated hydraulic-conductivity values. The results of a literature review of published approaches to the scaling of thermal and mechanical properties is included in annotated bibliography form as an appendix to this report. Conclusions from these numerical experiments 
have been implemented in the 1995 ground-water travel-time modeling exercise (GWTT-95) being conducted by the Yucca Mountain Project.

\section{Application of Scaling Methods to Yucca Mountain}

\section{Brief History}

Many of the early performance-assessment modeling studies for Yucca Mountain have ignored the issue of scaling material properties. Most of the flow-and-transport calculations used in these early studies have involved one-dimensional hydrologic models, and some of these models have simply used so-called "representative" material properties generally derived from some type of averaging of the available laboratory property measurements (Dudley and others, 1988; Barnard and Dockery, 1991). Other studies that have attempted to address uncertainty issues (Barnard and others, 1992; Kaplan, 1993; Wilson and others, 1994) have used material-property values sampled randomly from univariate probability distributions, without spatial correlation. Generally the probability distributions from which these Monte-Carlo property values are obtained were based either on assumptions of a Gaussian distribution characterized by a mean and a variance (Lin and others, 1986; Rutherford and others, 1992), or, for most later work, on the beta distribution (Barnard and others, 1992; Wilson and others, 1994). The beta distribution offers a greater degree of customization of the shape of the probability distribution (Kaplan and Yarrington, 1989; Kaplan, 1991).

The first major effort at Yucca Mountain to scale material-property values measured on core-sized specimens to values appropriate for large computational blocks was reported by Wilson and others (1994). This total-system performance assessment modeling exercise was based principally upon univariate variability of material properties, and the required hydrologic property values were generated by sampling from beta probability density functions. However, the variability of those probability functions was adjusted to reflect decreasing variance with increasing scale. These authors used a formula for adjusting the coefficient of variation $(\mathrm{CV}=$ standard devi- 
ation divided by the mean) originally developed by Freeze and others (1990): $S C V=C V \sqrt{\lambda / \bar{t}}$, where $S C V$ is the scaled coefficient of variation, $\lambda$ is the vertical range of spatial correlation, and $\bar{t}$ is the average thickness of the specific hydrologic unit. The vertical correlation length, $\lambda$, is assumed to be greater than the unit thickness, $\bar{t}$, with the result that the scaled coefficient of variation is smaller than the unscaled equivalent. Although this methodology does not change the mean, or expected value, of the probability distribution, scaling the coefficient of variation does reduce the spread of the distribution from which the actual values used in the Monte Carlo evaluation of the hydrologic flow-and-transport models are derived. Scaling of the coefficient of variation neglects in the influence of spatial continuity in the stratigraphically horizontal dimension. However, the main flow analyses conducted by Wilson and others were one dimensional and vertical.

\section{Purpose of "Scaling"}

The need for scaled parameters, or material property values, originates from the computational requirements of numerical flow or flow-and-transport modeling methods that discretize the model domain. Most practical applications of numerical physical-process modeling require discretization at a scale coarser than the scale at which material-property information can be measured. Some technique must be used to convert the measured data to a form that adequately represents the underlying physical process of interest within the relevant numerical approximation of that process.

A distinction must be made between those conversion techniques that provide an effective parameter and those which provide an upscaled parameter. An effective parameter can be defined as one that becomes a material property of the medium. The scale of averaging is large enough, relative to the scale of local variability, that an "effective" value of the global parameter results. The averaging volume required to obtain an effective parameter is large enough to be insensitive to the surrounding boundary conditions. The effective property value is also insensitive to the specific location and shape of the averaging volume. Furthermore, the effective value is insensitive to 
further increases in the size of the averaging volume (Dykaar and Kitanidis, 1993). This is essentially the concept of a "representative elementary volume." In contrast, the term upscaling is generally used to refer to the more practical problem of populating grid blocks in numerical flow models with single values of a hydrologic or physical property. In upscaling, the volume over which the scaled property value is determined is defined by the discretization of the flow or flowand-transport model. Upscaled values generally are dependent on the local boundary conditions and on the spatial location of the averaging volume.

Much of the current interest within the Yucca Mountain Project is focused on the latter problem: upscaling properties in order to accomplish numerical modeling of physical processes, most specifically, hydrologic properties. Another consideration thus becomes the purpose of the hydrologic modeling. Most of the scaling techniques for defining a scaled value identified from the literature are based on reproducing global flux through a system. If, however, the problem is to determine accurately the first arrival times in a ground-water travel-time study, then the use of scaled properties that only reproduce global flux may be incorrect and misleading. For situations directed toward understanding travel times and mass transport, upscaling techniques that preserve high hydraulic-conductivity pathways at the coarser scale of discretization should be used or may need to be developed. Alternatively, if the scaling techniques used preserve bulk flux, adequate dispersion must be modeled to reproduce the actual travel times.

A major focus of the Yucca Mountain project is groundwater flow through the unsaturated zone. The hydraulic conductivity of a model cell is a fractional value of the saturated hydraulic conductivity and is a function of the degree of saturation. The value of saturated hydraulic conductivity for a given sample is used to define the relationship describing the hydraulic conductivity as a function of saturation. Because saturated hydraulic conductivity is easier to measure in the laboratory than the parameters defining the pressure-saturation relationship, saturated hydraulic conductivity can be used to estimate these parameters through regression relationships. 


\section{Site-Specific Considerations}

Measurements of hydrologic properties (such as porosity, bulk density and saturated hydraulic conductivity) obtained from samples at Yucca Mountain suggest that these properties are heterogeneous, yet correlated spatially (McKenna and Rautman, 1995; Istok and others, 1994). Spatial continuity patterns also appear strongly anisotropic. Anisotropy is caused principally by layering in the tuffaceous materials. Heterogeneity also is controlled by stratigraphic layering, which largely reflects depositional and cooling processes. Variations in physical and hydrologic properties generally are much greater between stratigraphic units than within units.

The unsaturated zone at Yucca Mountain arguably is of greater modeling interest than the saturated zone. Because a major portion of flow and potential radionuclide transport would occur under unsaturated conditions, it is relatively more difficult to define discrete flow units. This difficulty is compounded by the fact that many of the different stratigraphic units that have been described from Yucca Mountain exhibit gradational "contacts." In some modeling cases, it may be desirable to determine upscaled properties within discrete geologic units, which are, by definition, presumably relatively homogeneous. However, the more general case most likely will require techniques that are suitable for the scaling of material properties independent of the units from which those properties are obtained.

\section{Requirements of Scaling Algorithms for Use at Yucca Mountain}

Perhaps the principal site-specific criterion that must be satisfied for practical use of any scaling technique at Yucca Mountain is the ability to scale highly variable distributions of hydraulic conductivity accurately. $\log _{10}$ variances of hydraulic conductivity distributions measured on the core-plug scale (approximately $40 \mathrm{~cm}^{3}$ ) at Yucca Mountain are on the order of 1.0. Another major requirement is that the scaling methodology be capable of dealing with anisotropy in the material-property field. A number of modeling exercises for Yucca Mountain have been conducted that ignore or discount stratigraphic subdivisions and simply focus on the spatial distribu- 
tion of properties (Robey, 1993; 1994b; Rautman and Robey, 1993). A correlated requirement is that implementation of the scaling method or algorithm be fiexible. One proposed approach to the issues of gradational contacts, lack of definable flow units, and high degree of both anisotropy and heterogeneity has been to create modeling grids that adapt themselves to the local variability and heterogeneity of the material property of interest (Robey, 1994a).

Modeling of transport is important in addressing regulatory requirements for licensing a potential high-level nuclear waste repository at Yucca Mountain. “Transport” includes not only the transport of physical, sorbing and non-sorbing contaminants away from the repository region, but also transport that reflects the fastest travel times of conceptual, tracked particles to an arbitrary, regulatory boundary. Thus, useful scaling techniques must place at least equal emphasis on reproduction of the physical-transport behavior of the natural system. Incorporation of dispersionrelated phenomena unquestionably will be important.

\section{Description of Scaling Algorithms: A Literature Review}

An extensive literature search has identified a large number of published techniques and algorithms for computing both scaled and effective hydrologic properties. A number of studies have applied one or more of these proposed approaches to actual ground-water problems. This section presents a summary of these techniques and algorithms.

\section{Scaling of Porosity}

Porosity can be considered an additive, or bulk, property of the geologic medium in modeling most hydrologic and thermal processes. Because porosity is simply the fraction of void space within a given spatial volume, scaling of porosity can be accomplished by integrating the void fraction of all discrete subvolumes contained within the larger volume of interest. Assuming that a number of sample measurements are "representatively" distributed in space, upscaling of porosity is typically accomplished by averaging arithmetically the porosity measurements within the volume of interest to obtain an effective, bulk porosity value for that volume. Thus: 


$$
\Phi_{e f f}=\frac{1}{N} \sum_{i=1}^{N} \Phi_{i},
$$

where $\Phi_{\text {eff }}$ is the effective, bulk porosity value, and the $\Phi_{i}$ are $N$ individual measurements of porosity. If the $\Phi_{i}$ are spatially or otherwise biased, some method should be adopted to compensate for that bias (Journel, 1983; Deutsch, 1989a).

This intuitive approach of simple, arithmetic averaging has been used extensively in solving hydrological problems, even though rigorous proof of the concept has been lacking. Dagan (1979) examined the upscaling of porosity through effective-medium theory. Using a self-consistent approach, Dagan demonstrated that effective porosity for hydrologic modeling is defined by the arithmetic average for both uniform and gradually varying flows. Arithmetic averaging reproduces the mean value of the smaller-scale measurements at the larger scale (essentially by definition), and it also reduces the variance. This reduction in variance is intuitive, as extreme values are smoothed by averaging them with progressively more samples closer to the mean value at higher levels of upscaling.

Scaling techniques that consider the effects of spatial correlation have been developed in the mining industry. The distribution of ore grades at the scale of a mineable unit must be determined from much smaller samples obtained by exploratory drilling. This requirement has lead to recognition of a quantifiable relationship between sample volume and the variance of those sample measurements as a function of spatial correlation length. Clark (1979) presents a technique for estimating changes in the variance of a "point-scale" distribution when those values are combined to estimate the distribution of mined-block values. A point measurement is defined as the smallest scale at which samples are collected. The point-scale values are generally considered to be homogeneous and isotropic (i.e., no heterogeneity exits at any scale below that of the measurement). These techniques require only knowledge of the point-scale distribution and the point-scale correlation length to determine the variance of the distribution of larger-scale volumes. The scaling 
relationship has been validated many times in the mining industry by comparing the variability of mill output values with the variability of values predicted through development drilling.

\section{Scaling of Saturated Hydraulic Conductivity}

A number of authors have addressed the issue of developing upscaled or effective hydrologic properties from small-scale measurements. The resulting, proposed solutions can be subdivided into four major groupings. Analytical expressions are essentially forms of "averaging," and constitute the conceptually and computationally simplest techniques and the only techniques that can efficiently accommodate irregular grids. Numerical methods are effectively a set of inversemodeling techniques. Renormalization makes use of analogies between ground-water flow and the flow of electricity through a set of resistors. Finally, perturbation theory views a heterogenous medium as containing small-scale variability about a stationary mean hydraulic conductivity. The univariate and spatial distribution of this variability about the mean are used to determine analytical expressions for the effective hydraulic conductivity of the medium or of an numerical flowmodel grid block. Additionally, spatial averaging is a variant of an analytical expression which incorporates information on the spatial correlation of the property being averaged. Effective medium theory allows small, isolated inclusions composed of heterogeneous materials to be placed in an infinite continuum and an effective hydraulic conductivity value calculated for the system.

\section{Analytical Expressions}

The simplest methods of determining effective or upscaled hydraulic conductivity values are analytical expressions, essentially weighted averages. The arithmetic, geometric, and harmonic means are the most widely known of these averaging algorithms. These three averages can be shown to be special instances of a fourth, more general averaging technique: power-law averaging. 


\section{Geometric Mean}

The most common approximation for effective hydraulic conductivity is the geometric average of the small-scale hydraulic conductivity measurements within the relevant volume. The geometric average is given by:

$$
K_{G}=\exp \left[\frac{1}{N} \sum_{i=1}^{N} \ln \left(K_{i}\right)\right]
$$

where $K_{G}$ is the geometric mean saturated hydraulic conductivity, and the $K_{i}$ are the $N$ individual saturated-conductivity measurements. The geometric mean involves taking the natural logarithms of the hydraulic conductivity data, averaging them, and exponentiating the result.

The major problem with using the geometric average as an estimate of effective permeability is that the calculated value of the geometric mean can be dominated by a small number of low-value outliers in the data. The value returned by the geometric mean calculation is very sensitive to the low hydraulic conductivity values within the data set, whereas the actual effective hydraulic conductivity may be virtually independent of the low-valued outliers, especially in three-dimensional domains (Jensen, 1991). The physical process of flow is driven by the highconductivity values, particularly by connected high-conductivity values. In common with all of the analytical averaging algorithms, the geometric mean ignores spatial correlation.

A variant of the geometric average, the " $\mathrm{j}$ th Winsorized mean," has been suggested as an alternative to the traditional geometric mean for estimating effective hydraulic conductivity (Jensen, 1991). Calculation of the $\mathrm{j}^{\text {th }}$ Winsorized mean $\left(K_{j w}\right)$ involves ranking of all the data $\left(K_{i}\right)$ and then censoring the $j$ highest and $j$ lowest values and replacing them by $j$ times next highest or lowest values prior to calculating the geometric average (Jensen, 1991). Thus,

$$
K_{j w}=\exp \left\{\frac{1}{N}\left[j \cdot \ln \left(K_{j+1}\right)+\sum_{i=1+j}^{N-j} \ln \left(K_{i}\right)+j \cdot \ln \left(K_{N-j}\right)\right]\right\}
$$


In this manner, extreme values of the distribution do not contribute excessively to the geometric average, yet the replacement of the $2 j$ extreme values by their immediate, non-censored neighbors avoids weighting the geometric unduly toward only the median portion of the observed distribution. Note that when $j=0, K_{j w}$ becomes equal to $K_{G}$ from eq. 2 . Note also that the choice of $j$ is arbitrary. Presumably, $K_{j w}$ stabilizes at a near-constant value after extreme outliers have been omitted from the calculation but when $j$ is still relatively small.

In one of the pioneering studies of effective permeability, Warren and Price (1961) showed that the effective permeability of Monte-Carlo simulated permeability fields is the geometric mean of the small-scale values. The simulations of Warren and Price (1961) consisted of randomly generated permeability values; there was no spatial correlation beyond the length of the small-scale grid blocks.

\section{Harmonic Mean}

For one-dimensional flow problems, the effective hydraulic conductivity can be demonstrated to be equal to the harmonic average, $\mathrm{K}_{\mathrm{H}}$, of the small-scale values, and is given by:

$$
K_{H}=\frac{d}{\sum_{i=1}^{N} \frac{d_{i}}{K_{i}}},
$$

where $d$ is the total length of the flow path and the $d_{i}$ are the lengths of each individual flow segment with hydraulic-conductivities, $K_{i}$. In two- and three-dimensional layered systems, the harmonic mean also defines the effective hydraulic conductivity for flow perpendicular to the layering, as represented in figure $1(a)$.

\section{Arithmetic Mean}

Flow parallel to layering in a layered system has an effective hydraulic conductivity defined by the arithmetic average, $K_{A}$, weighted by the relative thicknesses of the individual layers. This geometry is illustrated in figure 1(b), and the computational formula is as follows: 


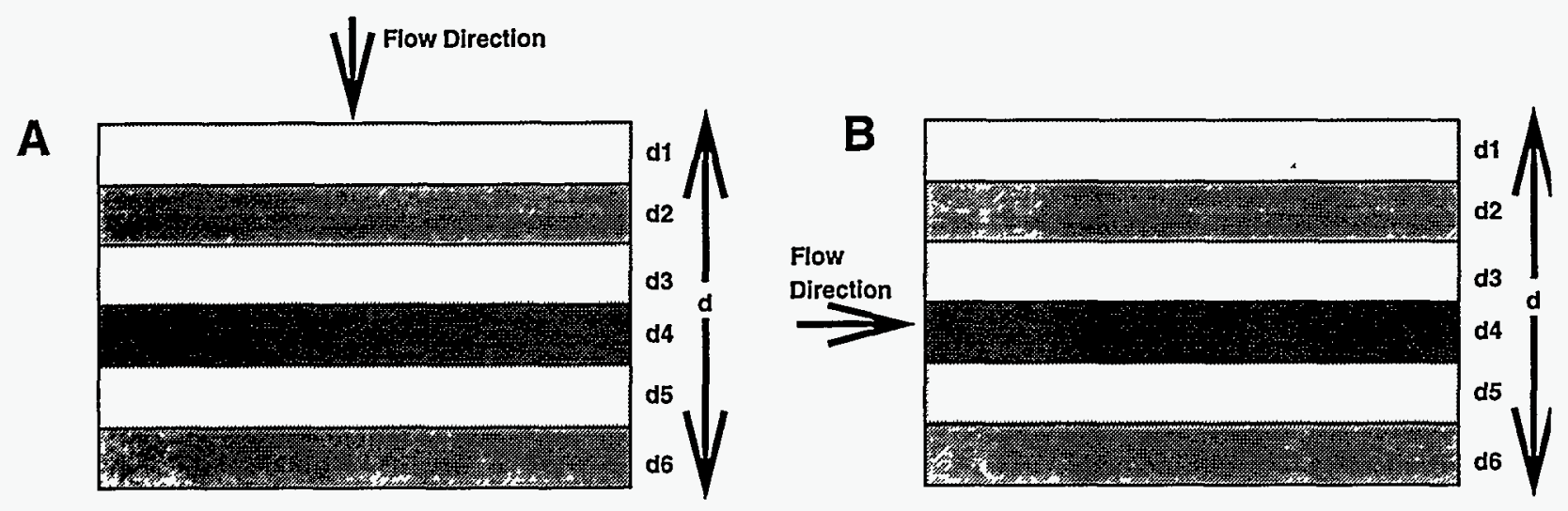

Figure 1. Schematic representation of flow (A) perpendicular and (B) parallel to layering in a twodimensional domain. Notation corresponds to that used in the equations in the text.

$$
K_{A}=\sum_{i=1}^{N} \frac{K_{i} d_{i}}{d}
$$

Symbols are the same as in equation 4. The two averages defined in equations 4 and 5 can also be referred to as the "series" and "parallel" formulations, respectively, for effective hydraulic conductivity. This naming convention comes from the analogy between hydraulic conductivity and electrical resistance.

\section{Bounding the Effective Conductivity Value}

The harmonic mean is the lower bound of effective hydraulic conductivity in two- and three-dimensional domains. Flow perpendicular to layering in a layered system is the most resistive to the transmittal of ground water; there is no way for flow to bypass any regions of low hydraulic conductivity values. The arithmetic mean is the absolute highest value of hydraulic conductivity possible in a two or three-dimensional system. Flow is channeled through the layers and, under the assumptions implicit in figure 1, each layer contains perfectly correlated values of hydraulic conductivity. Somewhere between these lower and upper limits lies the actual effective hydraulic conductivity. The geometric mean of the individual measurements also occurs between these limits. The simple geometric mean (equations 2 or 3 ) describes a system with no spatial cor- 
relation, whereas the harmonic mean (equation 4 ) describes a system with periodic correlation normal to the flow direction, and the arithmetic mean (equation 5) describes a flow system with perfect spatial correlation parallel to the direction of flow.

Recognition of the harmonic and arithmetic means as physical bounds on the values of effective hydraulic conductivity in a layered system has led to development another set of analytical expressions for effective hydraulic conductivity in systems that are not necessarily layered, but in which the flow direction is known. Duquerroix and others (1994) cite literature defining two limits bounding the effective hydraulic conductivity in two-dimensional ( $x$ and $y$ ), discretized flow domains. For flow in the $x$ direction, the maximum effective hydraulic conductivity $\left(K^{+}\right)$is obtained by computing the arithmetic average hydraulic conductivity for each column (for $x=\mathrm{a}$ constant) and then taking the harmonic mean of those arithmetic means. For flow in the same $x$ direction, the minimum effective hydraulic conductivity $\left(K^{-}\right)$is obtained by first computing the harmonic average for each row (for $y=$ a constant), and then calculating the arithmetic average of those harmonic averages. An obvious limitation of this technique is the requirement that the hydraulic-conductivity grid must be aligned with the principal flow direction.

These maximum and minimum hydraulic conductivity limits $\left(K^{+}\right.$and $\left.K^{-}\right)$defined by Duquerroix and others (1994) can be used as bounds for the value of $K_{e f f}$ These values also have been incorporated into expressions for $K_{\text {eff }}$ (Le Loc'h, 1989; Duquerroix and others, 1994). The expression presented by Le Loc'h (1989) is simply the geometric mean of the maximum and minimum $K$ values:

$$
K_{e f f}=\sqrt{K^{+} K^{-}}
$$

\section{Power-Law Averaging}

The arithmetic, geometric, and harmonic means may be considered special cases of a more general analytical function known as power-law averaging. Power-law averages have been 
used extensively in the oil industry to estimate the effective hydraulic conductivity of simulator grid blocks from core-scale measurements (Journel and others, 1986; Deutsch, 1989b). Dimitrakopoulos and Desbarats (1993) incorporated a power-law average into a spatial averaging equation. In general, the equation for a power-law average is written as:

$$
K_{w}=\left[\frac{1}{N} \sum_{i=1}^{N} K_{i}^{w}\right]^{\frac{1}{w}},
$$

where $K_{w}$ is the power-law average for a specific power, $w$, and the $K_{i}$ are the $N$ individual values of conductivity to be averaged. The bounding values of the maximum and minimum hydraulic conductivity computed using equation 7 are obtained for $w=1$ and $w=-1$ respectively; these correspond to the arithmetic and harmonic means. The geometric mean is defined as $w=0$, although equation 7 must undergo limited expansion (Deutsch, 1989b), because the expression as written is indeterminate at $w=0$. The spatial correlation structure of the hydraulic conductivity data determines the actual value of $w$.

A technique to determine the correct value of $w$ has been demonstrated by several authors (Deutsch, 1989b; Journel and others, 1986). The basic procedure makes use of the dependency of $w$ on the spatial correlation structure. Several fields of conductivity values the size of the desired flow-model grid blocks are generated that exhibit the desired spatial structure, and the effective conductivities of these fields are computed through use of a numerical flow model. These values are viewed as the true effective hydraulic conductivities of the grid-scale model volumes. A curve-fitting technique then fits the $K_{w}$ function of equation 7 to those true values, and the value of $w$ associated with the best-fit curve is taken as the appropriate power for general application. The majority of studies involving power-law averaging have involved bimodal hydraulic conductivity distributions, such as occur in many clastic, sand-shale depositional sequences. In petroleum applications, the power, $w$, is typically defined as a function of shale content of the reservoir. 


\section{Spatial Averaging}

A number of expressions for averaging of a property that exhibits spatial correlation and can be described by a second-order, stationary, random variable have been developed (Desbarats and Dimitrakopoulos,1990; Dimitrakopoulos and Desbarats, 1993; Desbarats, 1992). These techniques are based on geostatistical averaging methods and are referred to as "spatial averages." An algorithm for spatial averaging in two-dimensional domains (Desbarats and Dimitrakopoulos, 1990) uses the geometric mean of the point-scale data and the variogram describing those data. The upscaled value for a flow model grid block is given as:

$$
K_{e f f}=K_{G} \exp \left(\frac{\sigma^{2}-\gamma(V, V)}{2}\right)
$$

Here the variance term, $\sigma^{2}$, refers to the variance of all point-scale values that lie within the larger scale block. The expression, $\left[\sigma^{2}-\gamma(V, V)\right]$, is the average covariance value of the natural log hydraulic conductivity values within the large-scale block. The term $\gamma(V, V)$ is the average value of the point-scale variogram within the larger, upscaled block.

Another permutation of spatial averaging, developed for three-dimensional domains, is an extension of power-law averaging (Dimitrakopoulos and Desbarats, 1993). The significant feature of this extension relative to the power-law averaging defined in equation 7 is recognition of the dependence of effective hydraulic conductivity on the scale of the grid block over which it is calculated. In the work of Dimitrakopoulos and Desbarats (1993), effective hydraulic conductivity for a grid block in a numerical flow model is approximated by:

$$
K_{e f f}=K_{w} \exp \left[\frac{1-w}{2}\left(\sigma^{2}-\gamma(V, V)\right)\right]
$$

where $K_{w}$ is the power average of the entire suite of the core-scale hydraulic conductivity measurements, $\sigma^{2}$ is the variance of the natural $\log$ transform of the core-scale measurements, $w$ is the power and $\chi(V, V)$ is the average variogram value of the lognormal, core-scale hydraulic conductiv- 
ity values within the grid-block.

\section{Renormalization}

Renormalization is a technique for determining effective hydraulic conductivity that has seen limited use relative to other upscaling techniques. In its general sense, renormalization involves the derivation of mapping functions, which can translate the physical properties of a medium observed at one scale to a larger scale. The mapping takes place from one discrete scale to another discrete scale. King (1989) used an analogy between hydraulic conductivity and electrical conductance as a mapping function for hydraulic conductivity across scales. Another mapping function developed by Piggott and Elsworth (1992) is the geometric mean calculated through a mapping function that describes the probability of locations containing non-conductive sites. The complexity of mapping functions in both cases limits application of the scaling algorithm to blocks composed of $2 \times 2$ squares of smaller blocks in two dimensions and of $2 \times 2 \times 2$ cubes of smaller blocks in three dimensions. Both of these studies consider only the case for which the range of spatial correlation is no larger than the grid-block dimensions (random structure); they also assume an isotropic field of small-scale conductivity values.

\section{Numerical-Inverse Modeling}

Numerical techniques (also referred to as inverse techniques) typically involve the numerical solution of Darcy's law over a domain composed of separate, internally homogeneous, hydraulic-conductivity elements. By applying constant-head boundary conditions to opposite ends of the domain and no-flow boundary conditions to the sides of the domain, a uniform flow field is created (figure 2(a)). Effective hydraulic conductivity for the entire domain is determined by dividing the volumetric discharge by the cross-sectional area of the domain perpendicular to the flow direction and multiplying by the inverse of the gradient. There are several issues pertinent to the use of numerical techniques for determining effective hydraulic conductivity. 
The boundary conditions of the flow domain are set up arbitrarily to force a uniform gradient across the domain. This geometry allows calculation of the effective hydraulic conductivity only in the direction of flow. To calculate effective hydraulic conductivity in another direction, the gradient must be rotated. It is possible to define the principal directions of the hydraulic conductivity tensor through these gradient rotations; however, this calculation can become computationally expensive.

Another issue involving the design of the flow system is the imposition of no-flow boundary conditions along the sides of the domain. These boundaries are an artifice necessary for computationally efficient calculation of effective hydraulic conductivity. However, these no-flow boundaries may impose an unrealistic condition on the flow system. One technique for avoiding this problem is to embed the domain over which effective hydraulic conductivity is being determined within a "skin" of flow elements, as illustrated in figure 2(b) (Gomez-Hernandez and Journel, 1994). Obviously there is a trade-off between accuracy in modeling flow and the computational cost of using large skin volumes.

A

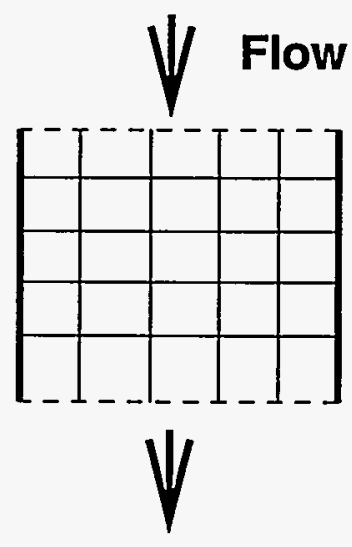

B

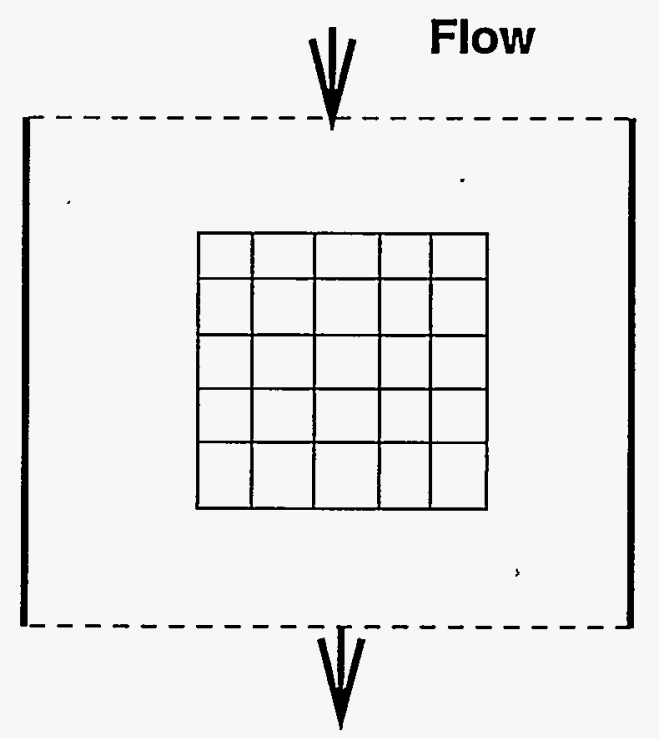

Figure 2. Conceptual representation of grids used in the numerical-inverse technique for upscaling. (A) Five-by-five grid of cells to be upscaled to a single block of size $5 \times 5$. (B) The same $5 \times 5$ grid embedded in a "skin" of flow elements. Bold lines parallel to the flow direction are no flow boundaries and the dashed lines perpendicular to flow are constant head boundaries. 


\section{Effective-Medium Theory}

The basis of effective-medium theory applied to hydraulic conductivity upscaling was defined by Dagan (1979). In his definition, a random assortment of blocks, each with an internally homogeneous hydraulic conductivity, is replaced with a single circle, or sphere in 3-D, with homogeneous hydraulic conductivity $K_{l}$. This circular inclusion is embedded in an infinite, homogeneous matrix with unknown hydraulic conductivity $K_{0}$ (Figure 3). It is possible to solve for $K_{0}$
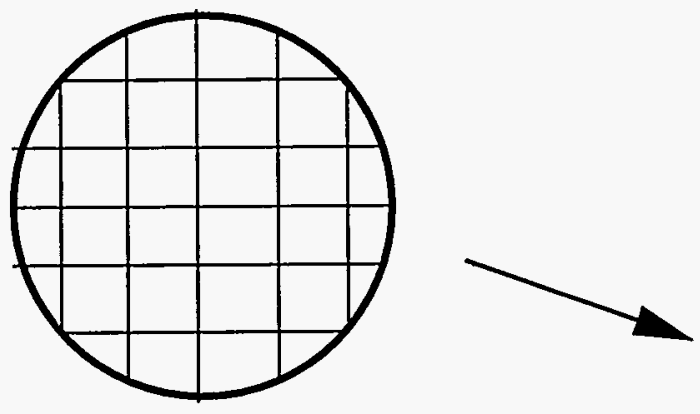

Figure 3. Conceptual representation of upscaling through effective-medium theory. A discretized domain of internally homogeneous blocks of hydraulic conductivity is replaced by a single isolated inclusion, with hydraulic conductivity equal to $K_{l}$, embedded in an infinite domain with hydraulic conductivity equal to $K_{0}$.

using analytic expressions for the pressure (head) field within the inclusion and within the surrounding matrix. By using these expressions, it is possible to solve for the mean head gradient and mean discharge in terms of the $K_{1}$ and $K_{0}$ (Dykaar and Kitanidis, 1992b). Dagan (1979) then used the self-consistent argument that the effective hydraulic conductivity $\left(K_{e f f}\right)$ is equal to $K_{0}$. For the case of a spherical inclusion, $K_{e f f}$ is given by:

$$
K_{e f f}=\frac{1}{m}\left[\int_{0}^{\infty} \frac{f(K)}{(m-1) K_{e f f}-K_{1}} d K\right]^{-1} .
$$


The dimension of the domain is denoted as $m$. The term $f(K)$ is the frequency function describing the gaussian distribution of the natural log hydraulic conductivity distribution. This function has been used previously in hydrological studies by Freeze (1975) and is given as:

$$
f(K)=\frac{1}{\sigma_{y}(2 \pi)^{1 / 2}} \exp \left[\frac{-\left(y-\mu_{y}\right)^{2}}{2 \sigma_{y}^{2}}\right],
$$

where $y$ is the natural $\log$ of $K, \sigma_{y}$ is the variance of the natural $\log K$ distribution, and $\mu_{y}$ is the mean of the natural $\log K$ distribution. The hydraulic conductivity given by equation 10 is not a function of the spatial correlation of the hydraulic conductivity data.

Dagan (1979) provides the results of solving equation 10 numerically in terms of the calculated $K_{\text {eff }}$ relative to the geometric mean hydraulic conductivity $\left(K_{G}\right)$. In two dimensions, the results of equation 10 are equal to the geometric mean. In three dimensions, the values of $\mathrm{K}$ provided by equation 10 is slightly greater than the geometric mean and becomes larger as the variability of the hydraulic conductivity distribution increases. Further work by Dagan (1989) has provided an analogous result for anisotropic cases by embedding an ellipsoid in the homogeneous matrix rather than a spherical inclusion. Limitations of the effective-medium theory are the requirement for a lognormal hydraulic conductivity distribution and the assumption that the inclusion embedded in the matrix does not interact with any other inclusions. This latter assumption can be more generally stated as there is no spatial correlation among heterogeneities.

Equation 10 has proven useful in a variety of effective hydraulic-conductivity studies. Desbarats (1987) performed a large number of numerical experiments in two-and three-dimensional domains containing a bimodal (sand/shale) hydraulic-conductivity distribution. The hydraulic conductivities considered by Desbarats were homogeneous within each facies. This homogeneity allowed a rather simple three-dimensional solution of equation 10 :

$$
K_{e f f}=\frac{1}{3}\left[\frac{V_{s s}}{2 K_{e f f}+K_{s s}}+\frac{V_{s h}}{2 K_{e f f}+K_{s h}}\right]
$$


where $V_{s s}$ and $V_{s h}$ refers to the volume fractions of the two facies, and $K_{s s}$ and $K_{s h}$ are the (uniform) hydraulic conductivities of sandstone and shale, respectively. Desbarats (1987) found that equation 12 agreed well with results of numerical flow modeling for shale fractions below 0.6 .

\section{Perturbation Theory}

Perturbation theory has also been used to examine the concept of effective hydraulic conductivity. Evaluation of heterogeneity by perturbation theory is based on the assumption that the hydraulic-conductivity field can be described by a mean value and "small" fluctuations about that mean; these small fluctuations have a mean of zero. The principal difficulty in applying the results of perturbation theory to defining effective hydraulic-conductivity values has been the definition of the small fluctuations in the hydraulic conductivity field. In the original work, estimates of effective hydraulic conductivity were found to be accurate when the standard deviation of the natural-log hydraulic conductivity values was less than or equal to 0.5 (Gutjahr and others, 1978). The results of work by Gutjahr and others (1978) are given by:

$$
\begin{array}{clc}
K_{\text {eff }}=K_{G}\left(1-\frac{\sigma_{y}^{2}}{2}\right) & \text { for 1-D flow } & \text { eq. } 13 \\
K_{\text {eff }}=K_{G} & \text { for 2-D flow, and } & \text { eq. } 14 \\
K_{\text {eff }}=K_{G}\left(1+\frac{\sigma^{2}}{6}\right) & \text { for 3-D flow. } & \text { eq. } 15
\end{array}
$$

The geometric mean hydraulic conductivity is denoted by $K_{G}$. These results can be compared to earlier work involving the geometric average of hydraulic conductivity. This work indicated that the effective hydraulic conductivity for steady flows was equal to the geometric mean in two dimensions, slightly below the that mean in one dimension and slightly above it in threedimensions. Further work has extended the validity of equations 13,14 and 15 to the case of gradually varying flow; that is, to cases where the scale of head fluctuations is large relative to the scale of hydraulic-conductivity fluctuations (Kitanidis, 1990). 
The development of equations 13,14 , and 15 relies upon several assumptions that may be restrictive depending on the problem. These assumptions are: (1) a natural-lognormally distributed hydraulic-conductivity field, (2) a locally isotropic hydraulic-conductivity tensor, (3) "small" fluctuations about the mean hydraulic conductivity, (4) an infinite domain, and (5) stationarity of the hydraulic-conductivity field.

Another application of perturbation theory to determine effective hydraulic conductivity has been presented by Duquerroix and others (1994). This work is an extension of equation 6 into anisotropic, two-dimensional domains and isotropic, three dimensional domains. To define effective hydraulic conductivity in an anisotropic medium, Duquerroix and others (1994) recognized that anisotropy can be caused by natural anisotropy inherent within the medium, or by the discretization of the domain for flow modeling. They defined anisotropy coefficients that take into account the anisotropy of the medium and its discretization:

$$
A_{y}=\frac{K_{y y}}{K_{x x}}\left(\frac{\Delta x}{\Delta y}\right)^{2} \quad A_{z}=\frac{K_{z z}}{K_{x x}}\left(\frac{\Delta x}{\Delta z}\right)^{2},
$$

where $A_{y}$ and $A_{z}$ are the anisotropy coefficients for the $y$ and $z$ directions relative to the x direction, and $d x, d y$, and $d z$ are the dimensions of the computational grid cells in the $x-, y$ - and $z$-directions respectively. The values of $K_{x x}, K_{y y}$ and $K_{z z}$ are the hydraulic conductivity values along the principal directions.

By approximation of a perturbation result, effective hydraulic conductivity in anisotropic two-dimensional domains is given by:

$$
K_{e f f}=\left(K^{+}\right)^{\Theta y}\left(K^{-}\right)^{(1-\Theta y)}
$$

where $K^{+}$and $K^{-}$are as defined for equation 6 , and $\theta y$ is given by:

$$
\Theta_{y}=\frac{\operatorname{atan} \sqrt{A_{y}}}{\pi / 2} .
$$


As $A_{y}$ goes to 1.0 , equation 17 becomes equivalent to equation 6 .

\section{Scaling of Unsaturated Hydrologic Properties}

Literature discussing the upscaling of unsaturated hydrologic properties is small relative to that for saturated hydraulic conductivity. This section presents a brief summary of some of the more important identified references.

An important property governing unsaturated flow is the capillary pressure/saturation relationship (also known as the moisture-characteristic curve or moisture-retention curve). This relationship is most often quantified by a fitting a model to the moisture-characteristic curve. Several models are available to fit to the data; the Brooks and Corey relationship and the van Genuchten function are the most common. Previous performance-assessment calculations within the Yucca Mountain Project have approximated the moisture-characteristic curves with a gamma distribution (Robey, 1994a).

Several different numerical approaches have been employed to determine the change in the moisture-characteristic curve over a range of scales. Ferrand and Celia (1992) modeled drying and wetting at the pore scale using a percolation technique. Keuper and McWhorter (1992) also used a percolation model to examine the scaling relationship of moisture-characteristic curves. Their work extended the percolation model beyond the pore scale to look at drying and wetting in heterogeneous formations. At the largest scale, Desbarats (1994) devised a technique for estimating the moisture-characteristic curve at the formation scale in heterogeneous media under capillary-gravity equilibrium (static conditions).

An experimental approach to examining upscaling of unsaturated hydrologic properties was reported by Flint and others (1994), who worked with the unsaturated property known as sorptivity. Sorptivity defines the initial rate of imbibition in a one-dimensional wetting process without gravity (Zimmerman and others, 1993). Sorptivity is not an intrinsic property of the media, but is process dependent. A technique for defining the hydraulic conductivity based on 
measurements of sorptivity and knowledge of the moisture-characteristic curve was presented by Zimmerman and others (1993). Flint and others (1994) showed that sorptivity measurements conducted on one scale could be used to predict values of sorptivity at a larger scale. However, to make an accurate prediction of sorptivity at the larger scale, it is necessary to have characterized the spatial variability of sorptivity within that larger volume. Experimental work is currently underway at Sandia National Laboratories to develop methods for scaling sorptivity (Tidwell, in press).

\section{Evaluation of Proposed Scaling Algorithms: Numerical Experiments}

This section presents the results of several numerical experiments involving modeling problems typical of those anticipated in the modeling of Yucca Mountain. The principal emphasis is on scaling algorithms that have been identified as practical for populating flow-model grid blocks with appropriate material-property values. The numerical experiments involve two properties: porosity and saturated hydraulic conductivity. Scaling of unsaturated flow properties is not attempted in this study.

For porosity, we document the already well-known arithmetic nature of scaling and the associated reduction of variance of the upscaled property distributions. For saturated hydraulic conductivity, we compare the modeled flow-and-transport performance of detailed ("point-scale") material-property distributions with the performance of the same system for which the point-scale values have been upscaled by varying degrees. The experiments with hydraulic conductivity involve two separate modeling exercises. The first exercise examines the scaling of isotropic, spatially correlated fields as a function of scaling methodology and the degree of heterogeneity. The goal is to identify those scaling algorithms that perform well for highly heterogeneous (large variance) property fields, such as those identified at Yucca Mountain. To some extent, this numerical experiment corresponds to quantifying the behavior of the different scaling algorithms if the requirement is to scale hydraulic conductivity within identifiable, individual flow units. Observed variances of $\log _{10}$ hydraulic conductivity within these units range from approximately 0.5 to more 
than 1.0. The ranges of spatial correlation observed within individual units at Yucca Mountain range from approximately $15 \mathrm{~cm}$ (Tidwell, 1994) to thousands of meters (Istok and others, 1994; Wilson and others, 1994).

The second exercise focuses on the application of a single scaling technique, power-law averaging, to a simulated conductivity field similar to the inferred fields of this material property at Yucca Mountain. A representative, strongly anisotropic, east-west cross section of Yucca Mountain has been developed using data from borehole UZ-16. The goal of this exercise is to identify the appropriate power coefficient(s) for use in modeling ground-water travel time at Yucca Mountain. These experiments examined power-law averaging exclusively, because this scaling technique is efficient and flexible enough to handle the anticipated, irregular grids that are the output from adaptive-gridding programs that minimize internal heterogeneity (for example, Robey, 1994a).

\section{Scaling of Porosity}

The scaling of porosity through simple, arithmetic averaging is well documented as well as intuitive. This averaging technique will be used to scale porosity data for use in flow-and-transport modeling of Yucca Mountain, and the variability of those upscaled porosity values can be computed directly. However, a simple numerical experiment was conducted to demonstrate that the reduction in variability of scaled porosity values can be predicted accurately using the volume-variance technique described by tables in Clark (1979) without explicit upscaling.

\section{Experiment Summary and Results}

A synthetic, two-dimensional data set consisting of 65,536 point-scale porosity values was generated using the gaussian simulation program SGSIM (Deutsch and Journel, 1992). The simulated point-scale values have a mean of 0.22 , a standard deviation of 0.057 and a spatial correlation modeled by a spherical variogram with an isotropic range of $1 / 64$ th of the domain size. These point-scale values were progressively upscaled to five discrete, larger scales by doubling the size 
of a moving, conceptual square enclosing the point-values. Predictions from the volume-variance relationship were compared to the results obtained by averaging the point-scale measurements and calculating the variance of the distribution of averaged values for each level of upscaling (table 1). Although a formal performance measure consisting of the percent error of the predicted variability compared with the variability observed by actually computing the upscaled values is not presented, inspection of table 1 indicates that the comparison is trivial. With the exception of the two largest levels of scaling, the differences in standard deviation are exactly zero to three decimals (tenths of a porosity percent). These latter two levels differ by only 0.001 .

Table 1: Comparison of standard deviations predicted by the volume-variance relationship of Clark (1979) and the actual standard deviation of larger-scale blocks computed by arithmetic averaging

[Volume units are arbitrary, porosity is stated as a fraction; leaders (--) mean not applicable]

\begin{tabular}{ccc}
$\begin{array}{c}\text { Upscaled } \\
\text { Volume }\end{array}$ & $\begin{array}{c}\text { Predicted } \\
\text { Standard Deviation }\end{array}$ & $\begin{array}{c}\text { Actual } \\
\text { Standard Deviation }\end{array}$ \\
\hline 1 (point-scale) & -- & 0.057 \\
4 & 0.045 & 0.045 \\
16 & 0.033 & 0.033 \\
64 & 0.020 & 0.020 \\
256 & 0.010 & 0.011 \\
1024 & 0.006 & 0.007 \\
\hline
\end{tabular}

\section{Scaling of Saturated Hydraulic Conductivity}

Seven scaling techniques identified from the literature review were used in numerical experiments to quantify their performance with respect to upscaling isotropic, spatially correlated fields of hydraulic conductivity. These techniques are listed in table 2. Criteria for selecting the specific techniques included practicality of implementation, both in general and in the specific instance of application at Yucca Mountain.

The eighth technique listed in table 2, numerical-inverse modeling, is somewhat a special case. This scaling technique would not normally be implemented in the manner employed in the numerical experiments, as repeated application of Darcy's law to an exhaustive set of progressively larger grid blocks would serve no purpose, and indeed would be more computationally 
Table 2: Scaling Techniques Selected for Numerical Experimentation

\begin{tabular}{cccc}
\hline $\begin{array}{c}\text { Technique } \\
\text { Name }\end{array}$ & Principal References & $\begin{array}{c}\text { Equation Number } \\
\text { in this Report }\end{array}$ & $\begin{array}{c}\text { Abbrevia- } \\
\text { tion }\end{array}$ \\
\hline Geometric Mean & Gutjahr and others, 1978 & eq. 3 & GM \\
Harmonic Mean & Freeze and Cherry, 1979 & eq. 4 & HM \\
Arithmetic Mean & Freeze and Cherry, 1979 & eq. 5 & AM \\
Power Average & Journel and others, 1986 & eq. 7 & PA \\
Spatial Averaging & Desbarats and Dimitrakopoulos, 1990 & eq. 8 & SA \\
Perturbation Theory & Duquerroix and others, 1994 & eq. 17 & PT \\
Renormalization & King, 1989 & see King, 1989 & RN \\
Numerical Inverse & many & Darcy's Law & NI \\
\hline
\end{tabular}

expensive than simply numerically solving the point-scale flow field directly. However, application of the technique in this manner does provide a standard of reference for comparison to the other seven techniques.

An additional reason for evaluating the performance of the numerical-inverse technique applied to progressively upscaled grid blocks relates to alternative scaling approaches not considered in this report. These alternative material-property modeling approaches involve the direct simulation of upscaled conductivity fields. Rubin and Gomez-Hernandez (1990) and Indelman (1993) inferred the statistical moments of an upscaled property field using knowledge of the small-scale samples and the flow field. However, the specific techniques used by Rubin and Gomez-Hernandez and by Indelman are limited to isotropic, lognormal distributions of hydraulic conductivity. Gomez-Hernandez and Journel (1994) and Tran (1994) extended the direct-generation concept in a more practical manner. In this latter approach, a large field of small-scale conductivity values is generated using geostatistical simulation. Random block-scale volumes within that field are selected and upscaled using numerical-inverse flow modeling. The univariate statistics and spatial character of these upscaled blocks fields is then considered representative of the upscaled material property in general, and the statistical moments derived from these upscaled blocks (including the variogram) are used to generate multiple realizations of the upscaled property directly. Full discussion of approaches involving direct generation of scaled conductivity fields are beyond the scope of this report. 


\section{Empirical Approach and Definition of Performance Measures for Flow and Transport}

Point-scale, spatially correlated hydraulic conductivity fields were generated using sequential Gaussian simulation. Flow and transport were modeled through these point-scale fields, and the results of these calculations were assumed to be the "true" flow-and-transport results for each different field. For the experiments involving the isotropic, spatially correlated conductivity fields, the point-scale conductivity fields were then upscaled one level using the different scaling approaches listed in table 2. Flow and transport through the scaled fields were then modeled using the same boundary conditions, and the results tabulated for comparison with the true (point-scale) values. The scaled fields were then upscaled an additional level, and the flowand-transport calculations repeated. The experiment using the anisotropic conductivity field representing Yucca Mountain involved only a single scaling technique.

Measures for evaluating the performance of the different scaling techniques were defined as the reproduction of the point-scale, effective hydraulic conductivity of the entire model domain (for flow) and as reproduction of the point-scale ground-water travel time as determined through particle tracking (for transport). Secondary performance measures were defined as the modeled dispersivity, the median tortuosity value, and the computed Peclet number. These performance measures were computed both for the detailed, point-scale simulated hydraulic conductivity fields and for each of the progressively upscaled versions of those fields. The same performance measures were applied to the evaluation of both the isotropic conductivity fields and the anisotropic conductivity field similar to an actual cross-sectional profile of Yucca Mountain.

Steady-state flow was evaluated by determining the total flux through the domain using a ground-water model, MODFLOW (McDonald and Harbaugh, 1988) to solve the pressure field and the fluxes. Darcy's Law was then used to calculate the effective hydraulic conductivity of the domain:

$$
K_{e f f}=(Q / A)(\Delta l / \Delta h)
$$

eq. 19 
Travel times and particle tracks were determined through a semi-analytical interpolation technique (Pollock, 1988).

\section{Generation of Point-Scale, Isotropic, Spatially Correlated Conductivity Fields}

Two-dimensional, spatially correlated fields of saturated hydraulic-conductivity values were created using the sequential Gaussian simulation program SGSIM, version 1.40 (Deutsch and Journel, 1992). The first step in the creation of these conductivity fields involved the generation of unconditional, simulated fields that had a mean of zero and a variance of one. These point-scale fields were assigned arbitrary dimensions of 2,560 by $2,560 \mathrm{~cm}$. Each simulated point is assumed to represent a flow-model grid cell that is 10 units on a side, resulting in fields comprising 256 by 256 point values of conductivity. Seven different point-scale fields were produced with spatialcorrelation length varying from 10 arbitrary units (equal to the dimensions of the point-scale data dimensions) to 640 units (equal to one-fourth of the domain size). The spatial-correlation structure in all seven cases was assumed to be isotropic.

The second step converted these standard-normal $\left(\mu=0, \sigma^{2}=1\right)$ spatial arrays to fields possessing a specified mean and variance. Two different levels of $\log _{10}$ hydraulic-conductivity variability were used, corresponding to log standard deviations of 0.5 and $1.0\left(\sigma^{2}=0.25\right.$ and 1.0 , respectively). These two levels of variability are typical of the degree of variability encountered within units at Yucca Mountain (Wilson and others, 1994). A mean of $-4.0 \mathrm{~cm} / \mathrm{sec}$ was used for the distributions. The choice of a mean is rather arbitrary as all flow and transport results are presented relative to the results on the point-scale fields. These converted arrays of values were defined to represent $\log _{10}$ hydraulic-conductivity values, and the seven resulting point-scale conductivity fields are shown in figures 4, 5, 6 and 7 .

\section{Generation of Point-Scale, Anisotropic, Conductivity Field}

The Yucca Mountain Project is conducting a set of modeling exercises during 1995 that focus on regulatory performance requirements for a potential nuclear-waste repository with 

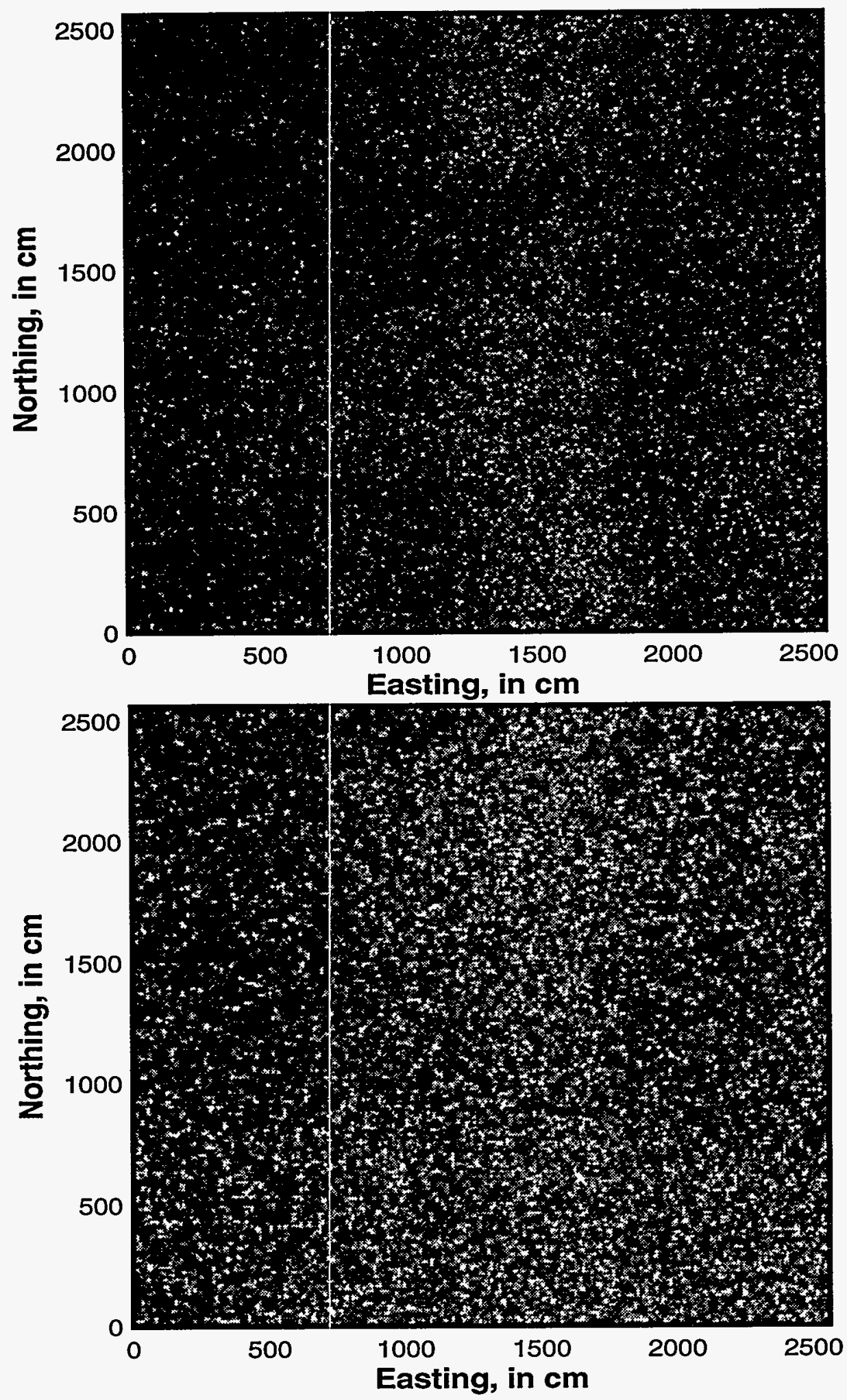

Figure 4. Grey-scale coded, 256-by-256, point-scale fields of log hydraulic conductivity: (top) isotropic spatial correlation of $10 \mathrm{~cm}$; (bottom) $20 \mathrm{~cm}$. Maximum value is white, minimum value is black 

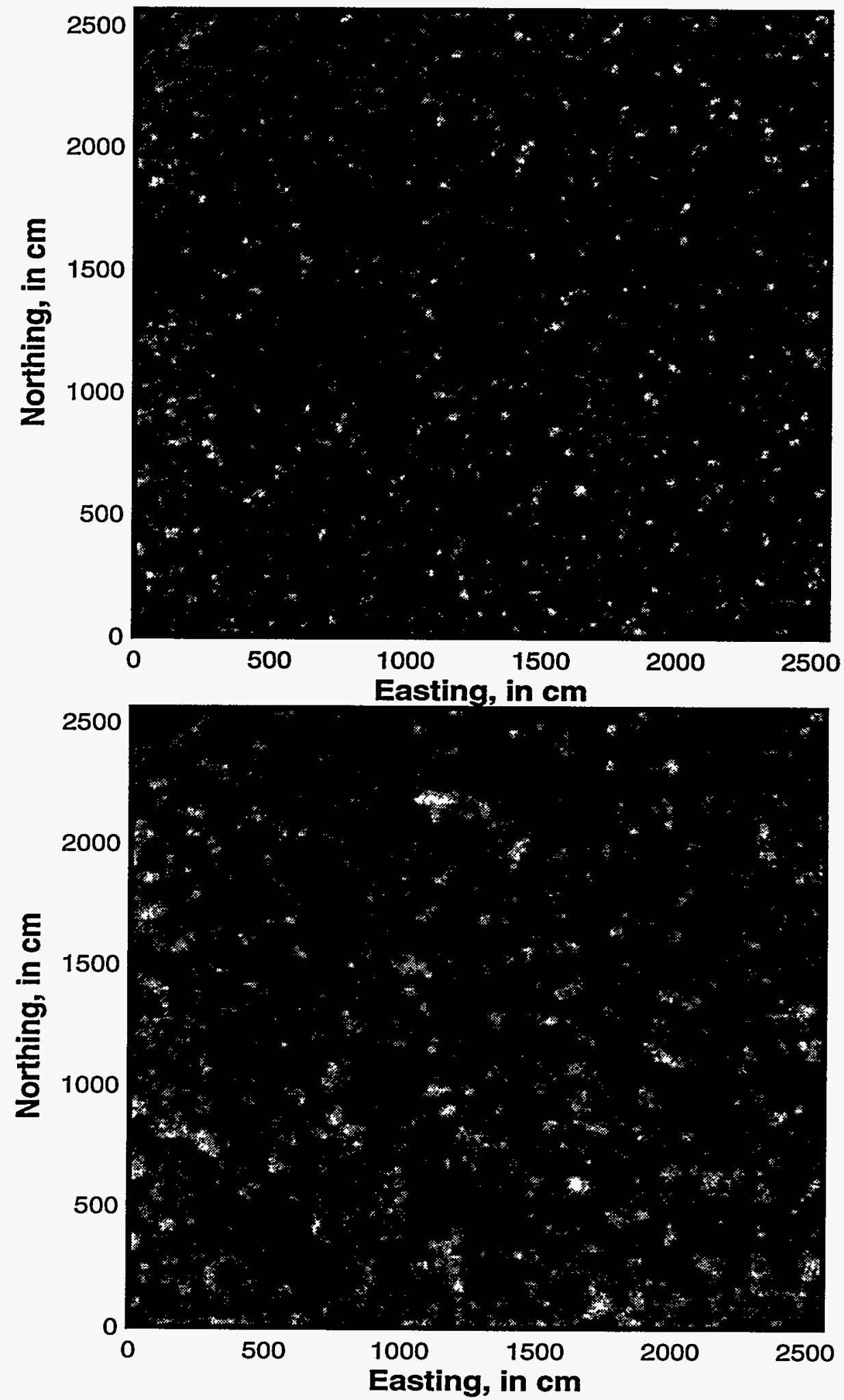

Figure 5. Grey-scale coded, 256-by-256, point-scale fields of log hydraulic conductivity: (top) isotropic spatial correlation of 40 $\mathrm{cm}$; (bottom) $80 \mathrm{~cm}$. Maximum value is white, minimum value is black 


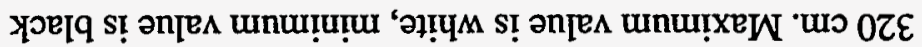

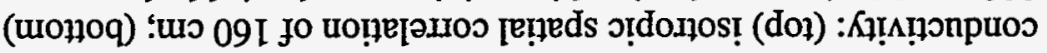

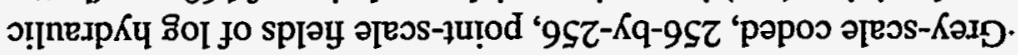

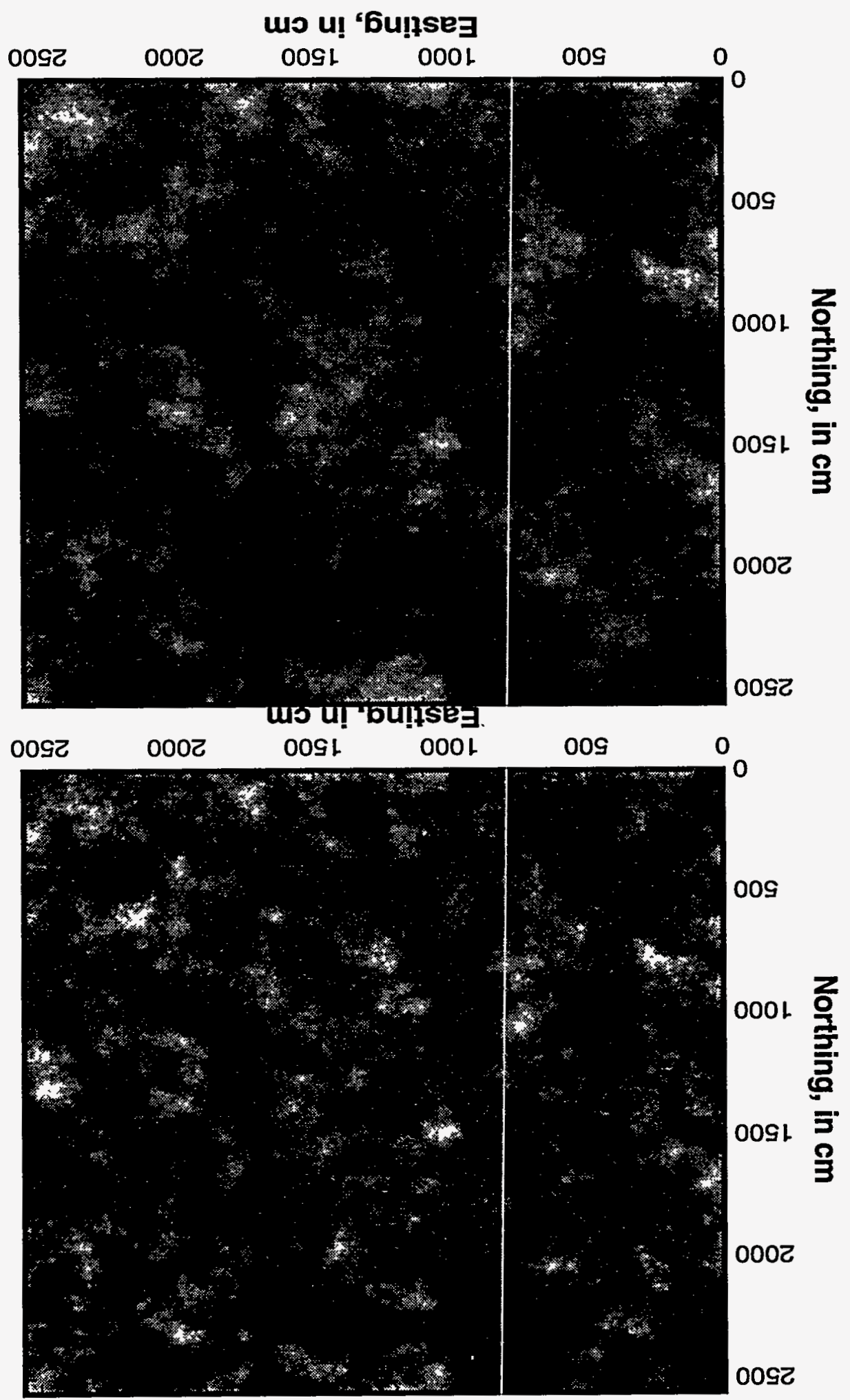




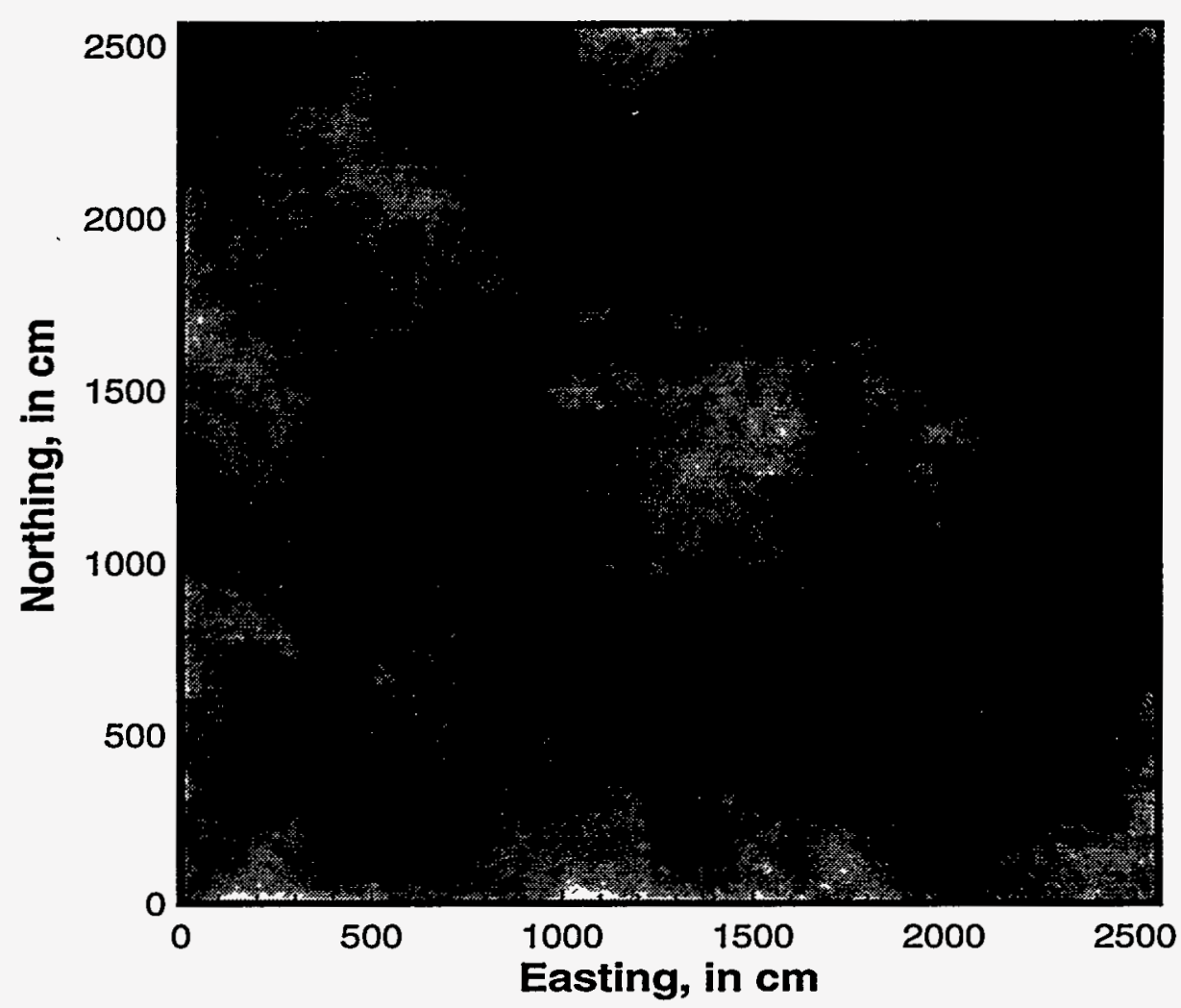

Figure 7. Grey-scale coded, 256-by-256, point-scale field of log hydraulic conductivity with isotropic spatial correlation of $640 \mathrm{~cm}$. Maximum value is white, minimum value is black.

respect to ground-water travel times. Known for convenience as "GWTT-95," these unsaturatedand saturated-zone flow calculations involve modeling several two-dimensional cross sections of Yucca Mountain. Ground-water travel time will be determined through flow-and-transport computations that involve particle tracking, in a manner similar to that evaluated in the more generic numerical experiments described in this report. Two-dimensional, geostatistical simulations of rock properties have been generated for Monte-Carlo-style input to the flow-and-transport calculational models.

The anisotropic simulated hydraulic-conductivity cross section of figure 8 was constructed using the process of linear coregionalization (Journel and Huijbregts, 1978). Porosity values from drill hole UE-25 UZ-16 (L.E. Flint, USGS, written communication, 1993) at Yucca Mountain 


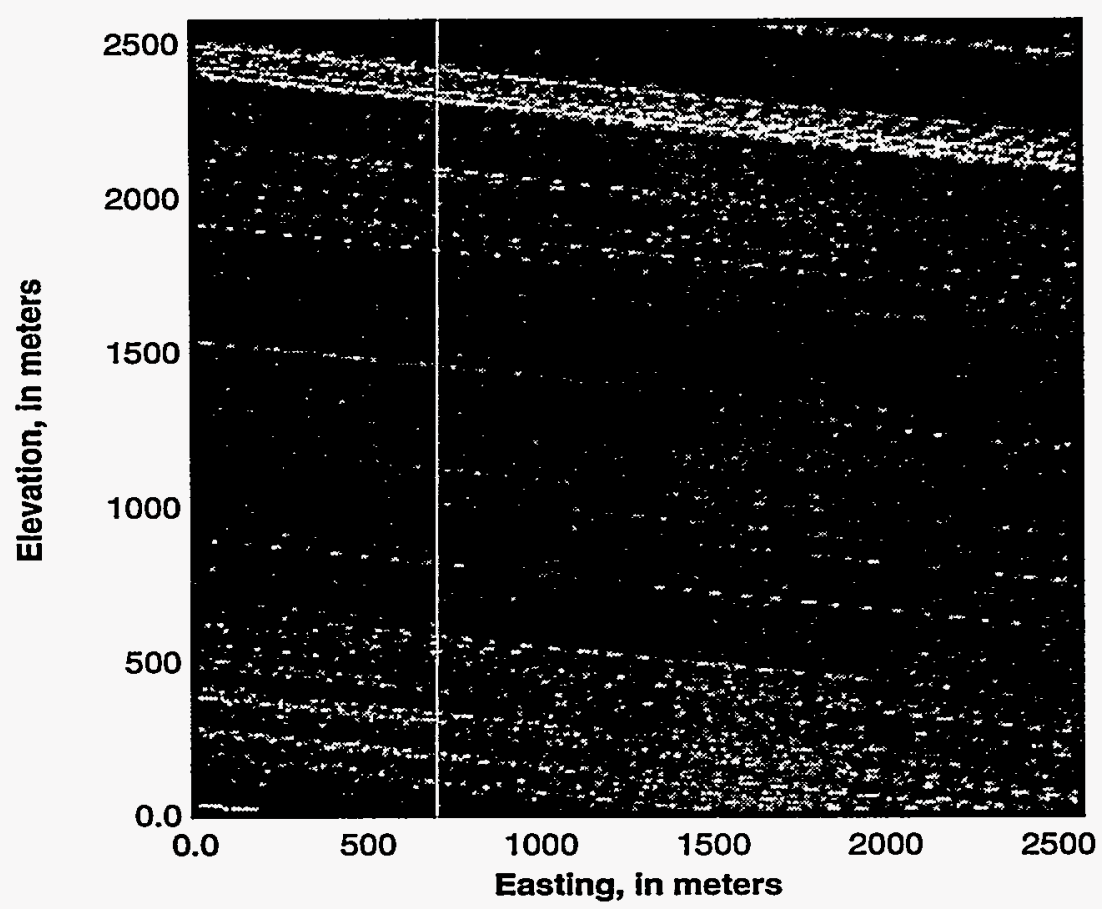

Figure 8. Simulated west-to-east, cross-sectional material-property model of Yucca Mountain for saturated hydraulic conductivity. Model has been created through a linear coregionalization of conductivity with porosity (Journel and Huijbregts, 1978). Values range from -12 (black) to -6 (white) $\mathrm{m} / \mathrm{sec}$ in $\log _{10}$ space.

were used to condition a Gaussian simulation of porosity (Deutsch and Journel, 1992). Linear coregionalization allows simulation of a secondary variable (here, log hydraulic conductivity) conditioned to the same variogram used to create the simulation of the primary variable (porosity) realization and to a linear-regression relationship between the two variables. The technique produces two spatially correlated fields that exhibit essentially the same cross-variable coefficient of determination $\left(r^{2}\right)$ as the conditioning linear regression. The technique is an alternative to "true" cosimulation of two variables that could be used when there are sufficient data from which to develop the required variograms and cross-variograms. The regression of log-hydraulic conductivity on porosity (and the corresponding $r^{2}$ value) was abstracted from surface outcrop data (Flint and others, 1996). 


\section{Scaling of Isotropic Conductivity Fields}

The point-scale, isotropic saturated hydraulic conductivity fields were scaled to progressively larger flow-and-transport model grids using the techniques listed in table 2 . In general, the scaling techniques were implemented by directly coding the relevant equation(s) for each scaling algorithm. Scaling was conducted as a series of $2 \times 2$ problems because the computations underlying the renormalization technique presented by King (1989) are complex and impractical for general implementation otherwise. Thus four point-scale values $(2 \times 2$ in $2-D)$ were upscaled into a single effective property, $K_{e f f}$ in a non-overlapping, moving-average process that encompassed the entire simulated conductivity field. To scale fields of permeability values larger than $2 \times 2$ through renormalization, the process is repeated, scaling four upscaled values into a single value over the entire field. For example, 64 small-scale values would be scaled four at a time to produce 16 values of $K_{\text {eff }}$ These 16 "larger small-scale" values would then be scaled four at a time into 4 values of $K_{e f f}$ and then finally these 4 values would be scaled to a single effective value representing the effective conductivity of the entire, original $64 \times 64$ simulated field.

Additional complexity is involved in the spatial-averaging technique (SA, table 2) proposed by Desbarats and Dimitrakopoulos (1990). This technique requires calculation of the average variogram value within each upscaled grid-block. The average variogram value $\bar{\gamma}$ in equation 8) was calculated using the Gauss-Cauchy algorithm with four Gauss points. Using larger numbers of gauss points may increase the accuracy of the solution. The specific algorithm used to calculate the average variogram value is a $C$ (version: HP-UX:92453-01A.09.61) translation of Fortran subroutine " $F$ " on pages 107-108 of Journel and Huijbregts (1978). The calculation of the average variogram value assumes that the coordinate axes of the upscaled grid-blocks are aligned with the axes of the spatial-correlation anisotropy ellipse. A second subroutine, which calculates the sum of multiply nested variogram structures in the relevant principal directions, has also been translated to $C$ from page 185 in Journel and Huijbregts (1978). 
The numerical-inverse scaling was accomplished by writing software to solve a blockcentered, five-point, finite-difference scheme on a square with an arbitrary number of internal elements. The solution of the Laplace equation is achieved by using Gauss-Seidel iteration with over-relaxation to minimize the change in head between iterations until a user-specified tolerance is reached. The head change tolerance used in this study was $5 \times 10^{-7}$ units ( $\mathrm{cm}$ or m) and a maximum of 100,000 iterations were possible.

\section{Scaling of the Anisotropic. "Yucca Mountain" Conductivity Field}

Scaling of the anisotropic hydraulic-conductivity field representative of Yucca Mountain was guided by consideration of requirements from the GWTT-95 modeling exercises. Preliminary plans for GWTT-95 call for the use of an irregular-gridding algorithm, which minimizes, in a global sense, the heterogeneity of the simulated hydraulic-conductivity values contained within each grid cell. The minimum-heterogeneity grid cells become the calculational elements for the flowand-transport models. Thus, a scaled value for each input rock property (here, hydraulic conductivity) must be derived for each flow-model element. The grid blocks developed using one possible irregular gridding algorithm are represented the polygons in figure 9 . For purposes of the numerical exercises, the conductivity cross section shown in figure 8 has been "stretched" vertically by a factor of 5.0. This adjustment was done to keep the aspect ratio of the upscaled grid blocks at 1:1, which is an approximation of the average aspect ratio of the irregular upscaled grid blocks in the adapted grid (Figure 9). This adjustment also allows scaling using square averaging cells, as in the numerical experiments involving the isotropic conductivity fields.

As long as the length of the flow-model calculational element is small relative to the length of spatial correlation of the material property being scaled $(L / \lambda$ approximately equal to 0.1 ), the numerical experiments involving the $2560 \times 2560-\mathrm{cm}$ simulated hydraulic-conductivity models indicate that all of the scaling methodologies perform equally well. The numerical experiments also indicate that performance of the different methodologies was more accurate for hydraulic conductivity fields with smaller overall variability (the fields with $\sigma=0.5$ ), compared to 


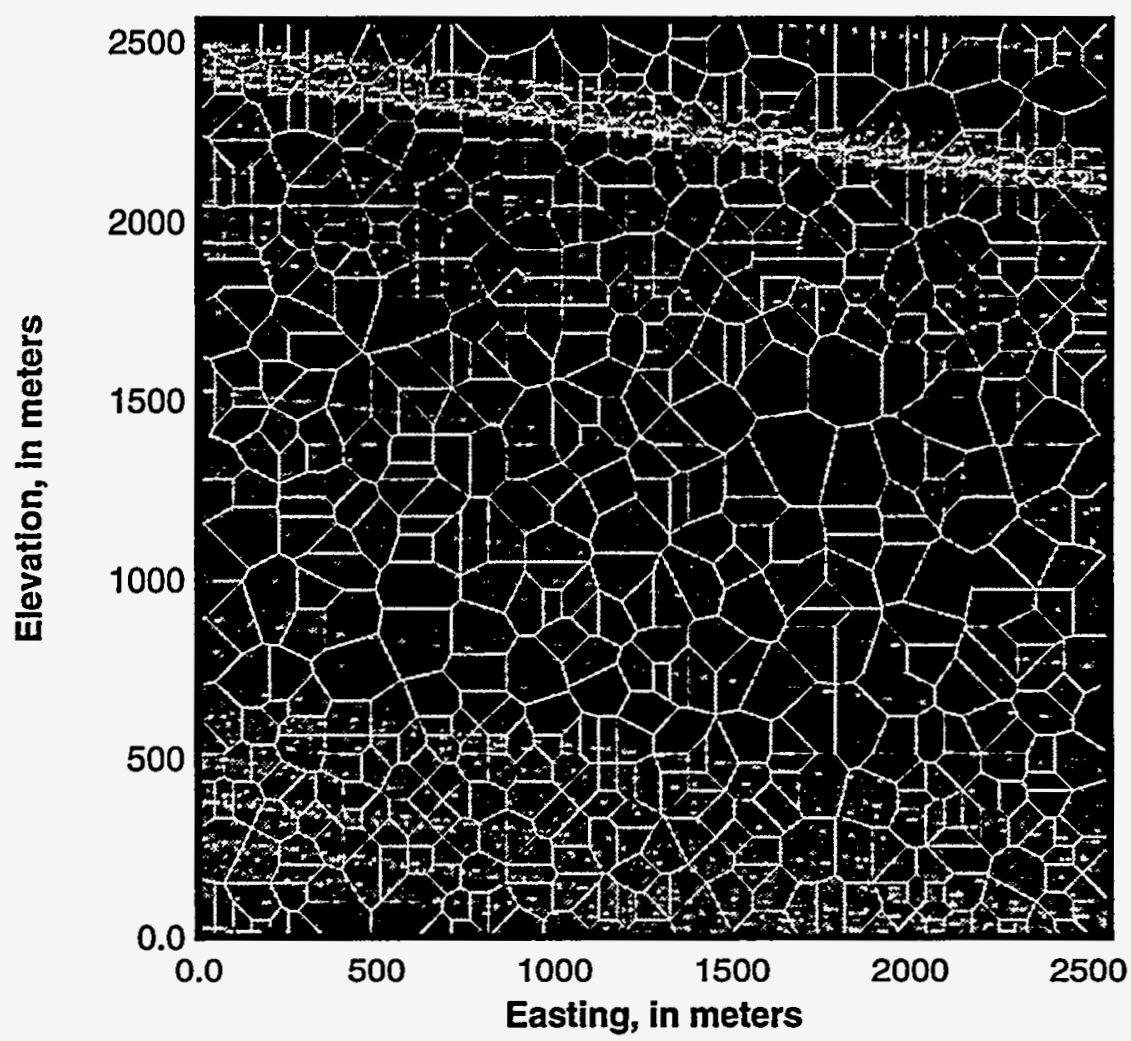

Figure 9. Irregular grid for the cross section shown in figure 8 that globally minimizes within-grid-cell material-property heterogeneity. The "quasi-homogenized" grid cells form calculational elements for the flow-and-transport model.

those with $\sigma=1.0$; figures 18 through 22). Use of heterogeneity-minimizing irregular gridding to define the calculational flow elements should reduce undesirable effects of scaling caused by large internal variations of material properties within those flow elements.

Practical, computational limitations effectively restrict the available scaling algorithms for use with irregular grids, like the one shown in figure 9 that minimizes internal material-property heterogeneity to some form of computationally simple averaging. The geometric average, provides a reasonable scaling algorithm in many instances and generally has been recognized as such for many years. However, when the geometric average is examined in light of the power-law equation (eq. 7), the geometric mean is nothing more than power-law averaging using an arbitrary power of $w=0$. Power-law averaging, in fact, provides a flexible means to implement all of the analytic expressions for scaling hydraulic conductivity. The exponent $(w)$ can be varied from -1.0 
(harmonic average) to 1.0 (arithmetic average), and the averaging expression should be customized for specific real-world problems through the use of non-integer values for $w$.

The concept of developing a custom scaling algorithm for Yucca Mountain using noninteger values of $w$ has been examined through numerical experimentation and comparison of selected flow-and-transport performance measures to the actual values of those measures obtained by computing the same measures using the point-scale hydraulic conductivity simulation (figure 8).

\section{Flow-Model Set Up}

The ground-water model MODFLow (McDonald and Harbaugh, 1988) was used to represent ground-water flow through the point-scale and upscaled simulated conductivity fields for both the isotropic and anisotropic models. In two dimensions; MODFLOW, uses a five-point, blockcentered, finite-difference scheme to solve the Laplace equation. A conjugate-gradient solver (Hill, 1992) minimizes the changes in both head and mass balance between iterations to a userspecified tolerance. The numerical experiments assumed a steady-state flow field with fixed heads at each end of the domain and with no-flow boundaries on each side (figure 2(a)). The constanthead boundary conditions specified a hydraulic gradient of 0.005 . A similar set-up was used to model flow through the anisotropic Yucca Mountain profile.

One hundred (100) particles were tracked from the top of the domain to the bottom using a semi-analytic velocity-interpolation technique implemented in MODPATH (Pollock, 1989). The particle-tracking algorithm works in conjunction with the block-centered, finite-difference scheme used in MODFLOW. Particles for particle tracking were released at the center of the top row of flow elements, and they were tracked to the top boundary of the bottom row of elements (Figure 7). The particle tracker records the coordinates of each particle whenever a particle enters a new flow element. Cumulative travel distance was calculated as the sum of the straight-line distances between points recorded by the particle tracker, and the velocity of each particle tracked 
through the domain was computed as the cumulative travel distance divided by the cumulative travel time. Note that because the flow-model grid blocks become larger with each progressive scaling step the transport domain becomes shorter and, the actual calculated travel times cannot be compared across the different levels of scaling. Instead, particle velocities are compared.

Longitudinal dispersivity and the dispersion coefficient were calculated based on the Peclet number. As employed in this study, the Peclet number is the ratio of advection to dispersion. The Peclet number $(P e)$ was determined through equation 3 of Moreno and Tsang (1994), modified here as:

$$
P e=\frac{1}{\left(\langle t\rangle\left\langle t^{-1}\right\rangle\right)^{1 / 2}-1}
$$

This equation is based on a one-dimensional solution of the advection-dispersion equation and provides a relationship between the first moment of the particle-arrival, $\langle t\rangle$, and inverse-particlearrival, $\left\langle t^{1}\right\rangle$, times and the Peclet number. The original equation 3 of Moreno and Tsang (1994) contained a slight error (C.-F. Tsang, Lawrence Berkeley Laboratory, personal communication, 1995). That error has been corrected in equation 20 .

From the Peclet number, both the longitudinal dispersion coefficient, $D_{l}$, and the longitudinal dispersivity, $\alpha_{L}$, are determined from the following relationships:

$$
\begin{gathered}
D_{l}=\left(v_{x} L_{f}\right) /(P e) \\
\alpha_{l}=D_{l} / v_{x},
\end{gathered}
$$

where $L_{f}$ is the macroscopic flow length and $v_{x}$ is the average velocity in the longitudinal flow direction $(x)$, calculated as the average velocity of the tracked particles. The interpolation algorithm (Pollock, 1989) used for the particle tracking has been shown to produce no artificial retardation (Ewing and Jaynes, 1995). Previous studies (Ewing and Jaynes, 1995, Goode and Shapiro, 1991) have shown that interpolation techniques for particle tracking are superior to mixing algorithms in 
terms of producing ensembles of particle travel times that give realistic values of retardation, diffusion and dispersion. Tortuosity was determined for each particle by dividing the cumulative distance, calculated as described above, by the macroscopic flow length $\left(\mathrm{L}_{\mathrm{f}}\right.$; the straight-line distance between the initial and final points of the tracked particles).

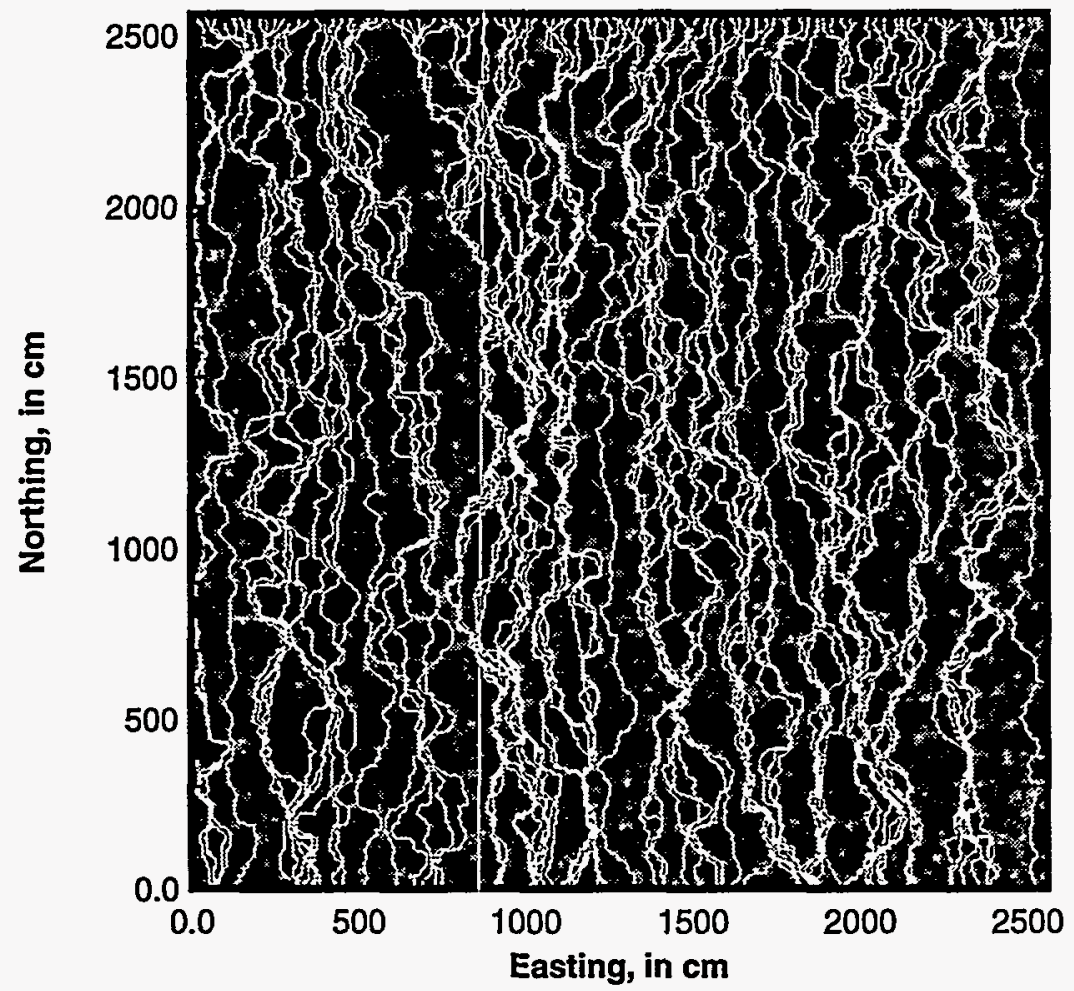

Figure 10. Example of 100 particle tracks through a heterogeneous point-scale log hydraulic conductivity field.

\section{Flow and Transport Results for Point-Scale, Isotropic Conductivity Grids}

The performance measures summarizing flow and transport results for the original, unscaled grids are shown in table 3. The corresponding travel-time distributions are shown in figure 11. These parameter values serve as the base case, against which the various upscaling techniques can be compared.

The effective hydraulic conductivity for the point-scale simulated fields with a log-conductivity standard deviation of 0.5 remains fairly stable from spatial correlation lengths of 40 to 

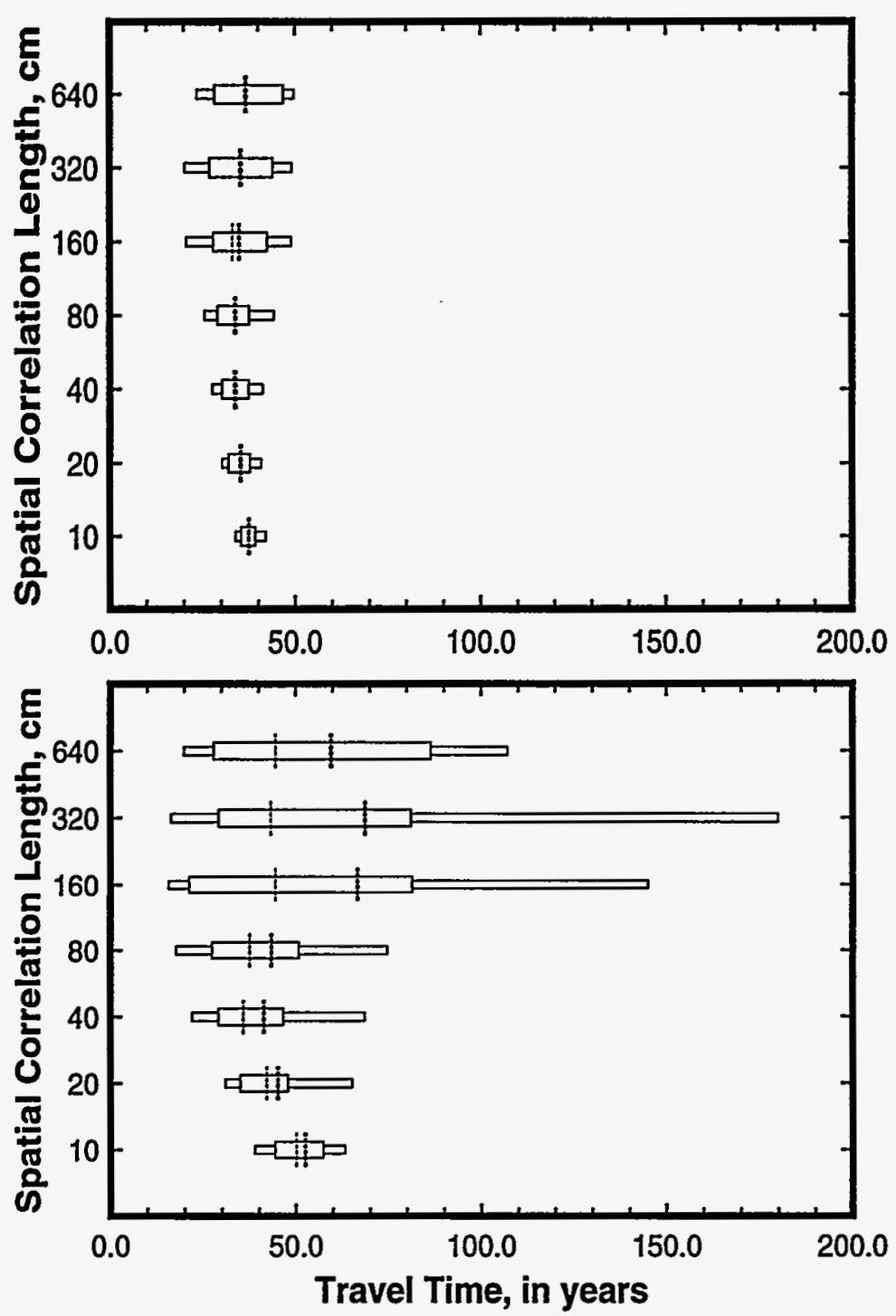

Figure 11. Box plots illustrating the distributions of arrival times for the seven pointscale realizations with progressively increasing ranges of spatial correlation: (a) standard deviation of $\log$ hydraulic conductivity equal to 0.5 ; (b) standard deviation of $\log$ hydraulic conductivity equal to 1.0 . The thick dashed lines represent the mean, the thin dashed lines represent the median, and the boxes define the 10th, 25th, 75th and 90th percentiles of the distributions.

640 units, as it does for the simulated fields with the higher standard deviation value. For spatial correlation lengths shorter than 40 units, the effective conductivity values are somewhat lower, as the shorter ranges of correlation allow individual, point-scale high (and low) conductivity values to be less structured and more disconnected from one another. However, above this range of correlation, all of the simulated models converge on the same effective conductivity value, approximately equal to -4.0 . The models with the smaller standard deviation appear to converge on this 
Table 3: Results of Flow-and-Transport Calculations for Original Point-Scale Grids Without Upscaling

\begin{tabular}{|c|c|c|c|c|c|c|c|}
\hline $\begin{array}{l}\text { Correlation } \\
\text { Length }(\lambda) \\
(\mathrm{cm})\end{array}$ & $\begin{array}{l}\text { Log Effective } \\
\text { Hydraulic } \\
\text { Conductivity } \\
\text { (cm/sec) }\end{array}$ & $\begin{array}{c}\text { Mean } \\
\text { Particle } \\
\text { Velocity } \\
\text { (cm/yr) }\end{array}$ & $\begin{array}{c}\text { Fastest } \\
\text { Particle } \\
\text { Velocity } \\
\text { (cm/yr) }\end{array}$ & $\begin{array}{c}\text { Dispersivity } \\
\text { (a) } \\
\text { (cm) }\end{array}$ & $\begin{array}{c}\text { Dispersion } \\
\text { Coefficient } \\
\left(D_{1}\right) \\
\left(\mathrm{cm}^{2} / \mathrm{yr}\right.\end{array}$ & $\begin{array}{l}\text { Median } \\
\text { Tortuosity } \\
\text { (unitless) }\end{array}$ & $\begin{array}{c}\text { Computed } \\
\text { Peclet } \\
\text { Number }\end{array}$ \\
\hline \multicolumn{8}{|c|}{ Standard deviation of $\log$ hydraulic conductivity $=0.5$} \\
\hline 10 & -4.07 & 70.9 & 87.3 & $\overline{8.3}$ & 588.5 & 1.04 & 305.2 \\
\hline 20 & -4.04 & 76.6 & 96.0 & 15.6 & 1195.0 & 1.05 & 163.6 \\
\hline 40 & -4.02 & 82.1 & 133.8 & 33.4 & 2742.1 & 1.06 & 76.3 \\
\hline 80 & -4.00 & 83.2 & 125.3 & 50.3 & 4185.0 & 1.06 & 50.6 \\
\hline 160 & -3.98 & 86.0 & 148.8 & 128.0 & 11008.0 & 1.08 & 19.9 \\
\hline 320 & -3.98 & 85.4 & 190.4 & 141.6 & 12092.6 & 1.06 & 18.0 \\
\hline 640 & -4.02 & 79.9 & 159.2 & 120.3 & 9612.0 & 1.05 & 21.2 \\
\hline \multicolumn{8}{|c|}{ Standard deviation of log hydraulic conductivity $=1.0$} \\
\hline 10 & -4.18 & 58.6 & 89.6 & 77.2 & 4523.9 & 1.14 & 32.7 \\
\hline 20 & -4.10 & 72.5 & 132.2 & 125.8 & 9120.5 & 1.17 & 20.2 \\
\hline 40 & -4.05 & 89.3 & 201.4 & 246.3 & 21994.6 & 1.21 & 10.3 \\
\hline 80 & -4.01 & 92.1 & 245.2 & 353.8 & 32585.0 & 1.21 & 7.2 \\
\hline 160 & -3.95 & 97.7 & 345.7 & 1143.3 & 111700 & 1.21 & 2.2 \\
\hline 320 & -3.94 & 95.1 & 382.9 & 1191.9 & 113350 & 1.24 & 2.1 \\
\hline 640 & -4.04 & 76.0 & 280.7 & 639.4 & 48594.4 & 1.16 & 4.0 \\
\hline
\end{tabular}

value at a slightly shorter range of spatial correlation than the models with the larger standard deviation. These results agree with those of Moreno and others (1988), who demonstrated that flow properties remained stable for cases where the spatial correlation length to flow model element length ratio $\left(\lambda / L_{e}\right)$ is greater than 2 for flow within a single, heterogeneous fracture plane.

Both the average travel time and the fastest travel time generally decrease as the spatial correlation length increases. This observation is consistent with the intuition that, as the range of spatial correlation increases, the high conductivity values within the simulated field will "tend" to become more connected, and thus to provide faster flow paths. Conversely, dispersivity increases with increasing spatial correlation. This increase occurs as some particles are advected in a fast, well-connected path and others do not enter that path. The tortuosity increases rapidly to a quasiconstant level. For both dispersivity and tortuosity, the changes with increased spatial correlation are more extreme for the simulated fields with the higher log-conductivity standard deviation as the channelization of particles becomes more pronounced. The Peclet number decreases markedly 
with increasing correlation; this effect is also more marked for the more variable (larger standard deviation) simulated fields.

Calculated dispersivities do not completely follow the analytic expression for dispersivity developed by Gelhar and Axness (1983), viz.:

$$
\alpha_{l}=\left(\sigma^{2} \lambda\right) /\left(\gamma^{2}\right)
$$

where $\gamma$ is a geometric term equal to 1.0 in isotropic media and $\sigma^{2}$ is the variance of natural log hydraulic conductivity (table 4). Equation 23 gives a reasonably accurate prediction of the modeled dispersivity for the point-scale fields with a correlation lengths below 40 (equal to $1 / 64$ th of the domain size) when the $\log _{10}$ standard deviation is only 0.5 . For the fields with the larger standard deviation of $1.0\left(\log _{10}\right.$ space), the expression from Gelhar and Axness underestimates the calculated dispersivity, until a range of 80 , after which the dispersivity is overestimated.

Table 4: Comparison of dispersivities calculated for the point-scale hydraulic conductivity fields and dispersivities predicted by the expression in Gelhar and Axness (1983).

[The equivalent natural $\log$ variance of the $\log _{10}$ field has been used]

\begin{tabular}{ccc}
\hline $\begin{array}{c}\text { Correlation } \\
\text { Length }(\lambda) \\
(\mathbf{c m})\end{array}$ & $\begin{array}{c}\text { Computed } \\
\text { Dispersivity } \\
\text { (cm) }\end{array}$ & $\begin{array}{c}\text { Predicted } \\
\text { Dispersivity } \\
\text { (cm) }\end{array}$ \\
\hline & Standard deviation of log hydraulic conductivity = 0.5 & \\
\hline 10 & 8.3 & 11.5 \\
20 & 15.6 & 23.0 \\
40 & 33.4 & 38.4 \\
80 & 50.3 & 92.0 \\
160 & 128.0 & 184.0 \\
320 & 141.6 & 368.0 \\
640 & 120.3 & 736.0 \\
\hline & Standard deviation of log hydraulic conductivity $=1.0$ \\
\hline 10 & 8.3 & 46.0 \\
20 & 15.6 & 92.0 \\
40 & 33.4 & 184.0 \\
80 & 50.3 & 368.0 \\
160 & 128.0 & 736.0 \\
320 & 141.6 & 1472.0 \\
640 & 639.4 & 2944.0 \\
\hline
\end{tabular}


There are several probable reasons for these discrepancies. The stochastic theory underlying the analytic expression for dispersivity (equation 23) assumes a natural-log hydraulic conductivity field of infinite extent described by an exponential covariance function. A spherical variogram model has been used in the numerical experiments because this expression for spatial continuity best reflects the available data from Yucca Mountain (McKenna and Rautman, 1995) and because this spatial model has been used previously to simulate material properties at Yucca Mountain (Rautman and Flint, 1992). Also, the simulated hydraulic conductivity fields are demonstrably not infinite in extent. The influence of finite boundary conditions unquestionably affects the calculated dispersivities shown in tables 3 and 4.

The amount of dispersion indicated by the dispersion coefficient in table 3 is seen to increase as the range of spatial correlation (ratio of $L_{f} / \lambda$ ) decreases. These results are consistent with the theory of asymptotic macrodispersion (Gelhar and Axness, 1983), under which the particles moving through the system must travel a distance equal to several correlation lengths before a stable dispersivity is achieved. At shorter travel distances, the dispersion process is non-Fickian, in other words, dispersion of the particles is a non-linear function of travel distance.

In a study of dispersion in a single heterogeneous fracture plane, Ewing and Jaynes (1995) found that as $L_{f} / \lambda$ increased, the amount of dispersion tended to zero. A decrease in dispersion as $L_{f} / \lambda$ increases is also noted in this study (table 3 ). These results are inconsistent with many field observations, which typically show dispersion increasing with travel distance (Gelhar, 1986). Possible explanations for this inconsistent behavior are the fact that only hydrodynamic dispersion was considered (no molecular dispersion), the numerical studies were two-dimensional whereas the field studies are three-dimensional, and the possibility that the aquifers studied in the field are fractal porous media such that the larger amounts of dispersion are caused by the particles progressively encountering larger and larger scale heterogeneities. Results of both this study and the fracture-plane study of Ewing and Jaynes (1995) suggest that dispersion is non-Fickian and that determination of a single asymptotic dispersivity value is not possible. 
The results of the tortuosity calculations (table 3) are consistent with those for the effective hydraulic conductivity. Beyond a $\lambda / L_{e}$ of 2 , the tortuosity values remain relatively constant across scales. The tortuosity values increase from the simulations generated with a log hydraulic conductivity standard deviation of 0.5 , which exhibit an average tortuosity of 1.06 , to an average tortuosity of 1.17 for the more variable conductivity fields with a log standard deviation of 1.0.

The exceptions to the intuitive statements of the preceding few paragraphs are also enlightening. Thus, whereas the effective conductivity values generally increase, as do dispersivity and tortuosity, and the travel times and Peclet numbers decrease with increasing spatial correlation, virtually all of these measures reverse their intuitive trend at the two largest correlation lengths. This tendency is most noticeable for the simulated fields with the range of spatial correlation set to $640 \mathrm{~cm}$.

The principal explanation for this anomalous, counter-intuitive behavior is found in the ergodic hypothesis of geostatistics. In practical terms, the ergodic hypothesis states that the measured statistical moments of a simulation, and in particular of an unconditional simulation, will tend toward the global expectations of those expectations of the random variable as the size of the simulation domain increases. There are various measures of the "size" of a simulation domain; however, a particularly relevant measure is the number of spatial correlation lengths that fit inside that domain. For the point-scale simulations, the domain size is initially 256 times the range of spatial correlation for $\lambda=10 \mathrm{~cm}$, and the domain decreases progressively to only four times the range of correlation for $\lambda=640 \mathrm{~cm}$. As the size of the domain decreases, the simulated models and the performance measures no longer conform to the ergodic hypothesis.

It is possible to examine the validity of the ergodic hypothesis for the simulated pointscale models of hydraulic conductivity. The point-scale models were generated using sequential Gaussian simulation. A fundamental property of the distributional model underlying this technique is the proportion of values that occur within one standard deviation of the specified, input mean value. For a true Gaussian distribution, 68.26 percent of the values lie within one standard 
deviation of the mean (Walpole and Myers, 1989). The fraction of values distributed within one standard deviation of the mean have been computed for the seven point-scale realizations with progressively increasing spatial correlation lengths. The results are shown in figure 12 . When there are few correlation lengths within the domain, the simulations deviate from the theoretical value of approximately 68 percent. As the number of correlation lengths within the domain increases beyond approximately 10 , the percentage of values within one standard deviation of the mean is close to the expected fraction.

Of the simulations reflecting the seven different correlation lengths, the field with $\lambda=640$ $\mathrm{cm}$ exhibits the largest deviation from the theoretical expectation. This field is excluded from further consideration as unacceptably nonergodic. The field with $\lambda=320 \mathrm{~cm}$ also deviates from the theoretical value; however, this deviation is only about two percent. This amount of error is deemed acceptable and this point-scale field has been retained for further analysis. It is this increase in the proportion of values near the mean, which translates to an unintentional decrease in variability, that causes the observed decrease in travel times and dispersivity at the largest spatial correlation lengths.

By simply examining the flow and transport parameters across the range of spatial correlation, the effects of upscaling can be examined. Although no upscaling process has been applied to the point measurement fields, the ratio of domain length $\left(\mathrm{L}_{\mathrm{D}}\right)$ to correlation length $(\lambda)$ decreases as $\lambda$ increases and the domain size remains constant. This is the type of upscaling examined by Ewing and Jaynes (1995). In contrast, the current report examines the merits of various techniques that can be used to increase the flow model grid-block length (also referred to as the element length, $\mathrm{L}_{\mathrm{e}}$ ) to spatial correlation length ratio.

\section{Flow-and-Transport Results for Scaled, Isotropic Conductivity Grids}

The seven simulated point-scale isotropic fields of saturated hydraulic conductivities with differing ranges of spatial correlation have been scaled to five discrete, larger scales. The degree 


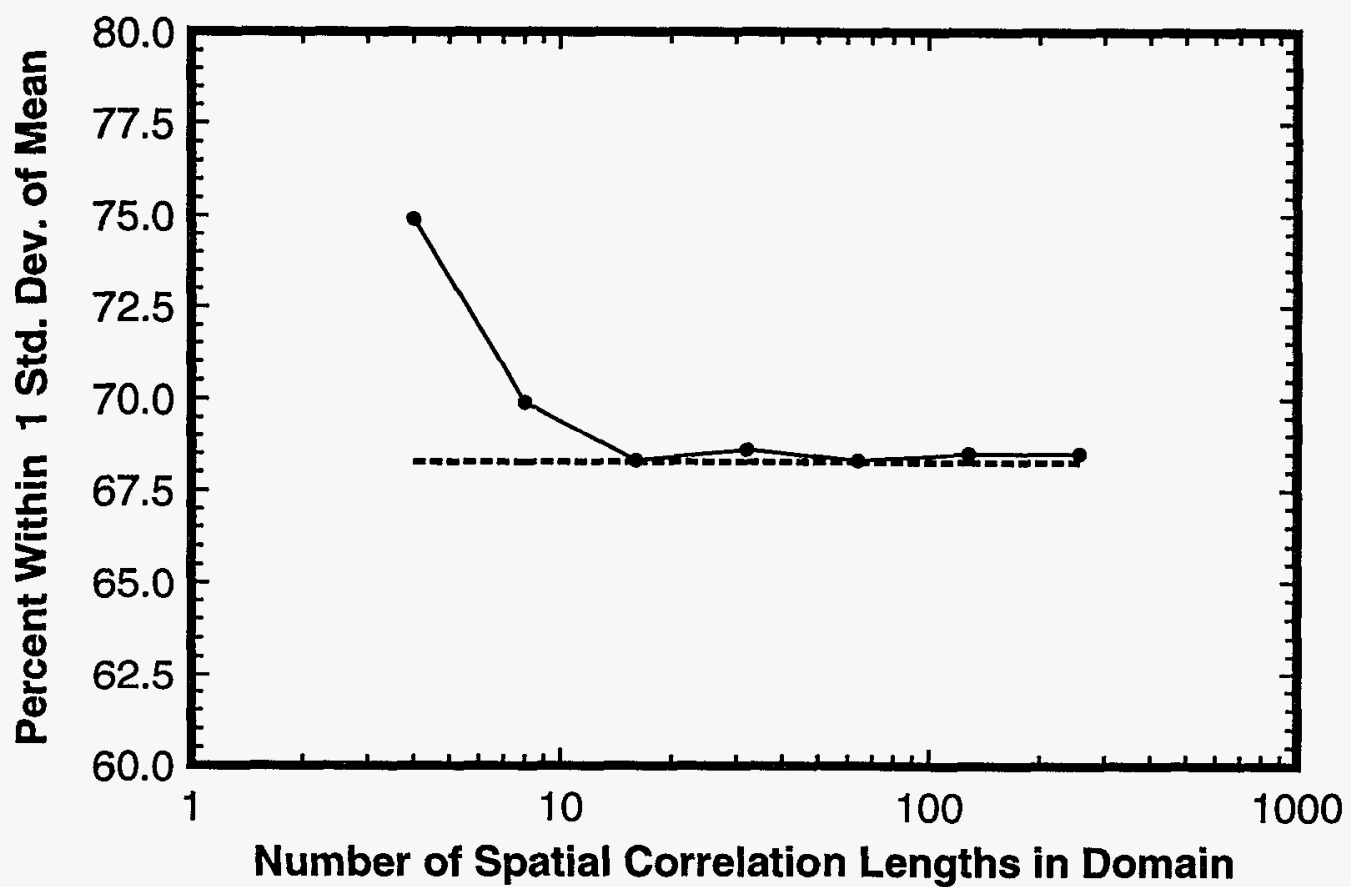

Figure 12. Percent of values in point-scale simulations that are within one standard deviation of the mean. The dashed line represents the theoretical value for a Gaussian distribution.

of scaling can be described in two ways. First, scaling can be described by the number of flowmodel elements used to discretize each side of the domain. Alternatively, the degree of scaling can be described by the number of point-scale measurements that are combined into a single flowmodel element. The original grids, which consist of 256 point-scale values of hydraulic conductivity in each direction, are considered the base case for the scaling evaluation. A set of scaled grids might then be described as having been scaled " 4 -into-1," or as consisting of " $128 \times 128$ flow-model elements," for the case where $2 \times 2$ subgrids of point-scale values are scaled to one flow-model calculational element. The requirement that the subgrids remain square (see discussion of Renormalization beginning on page 15) limits the size of the possible subgrids to powers of 2 (viz. 4, 16, 64, 256, 1024). These alternative descriptions of the five discrete levels of scaling are presented conceptually in figure 13. An example showing one point-scale, simulated conductivity field scaled to each of the five discrete scales is shown in figure 14. 


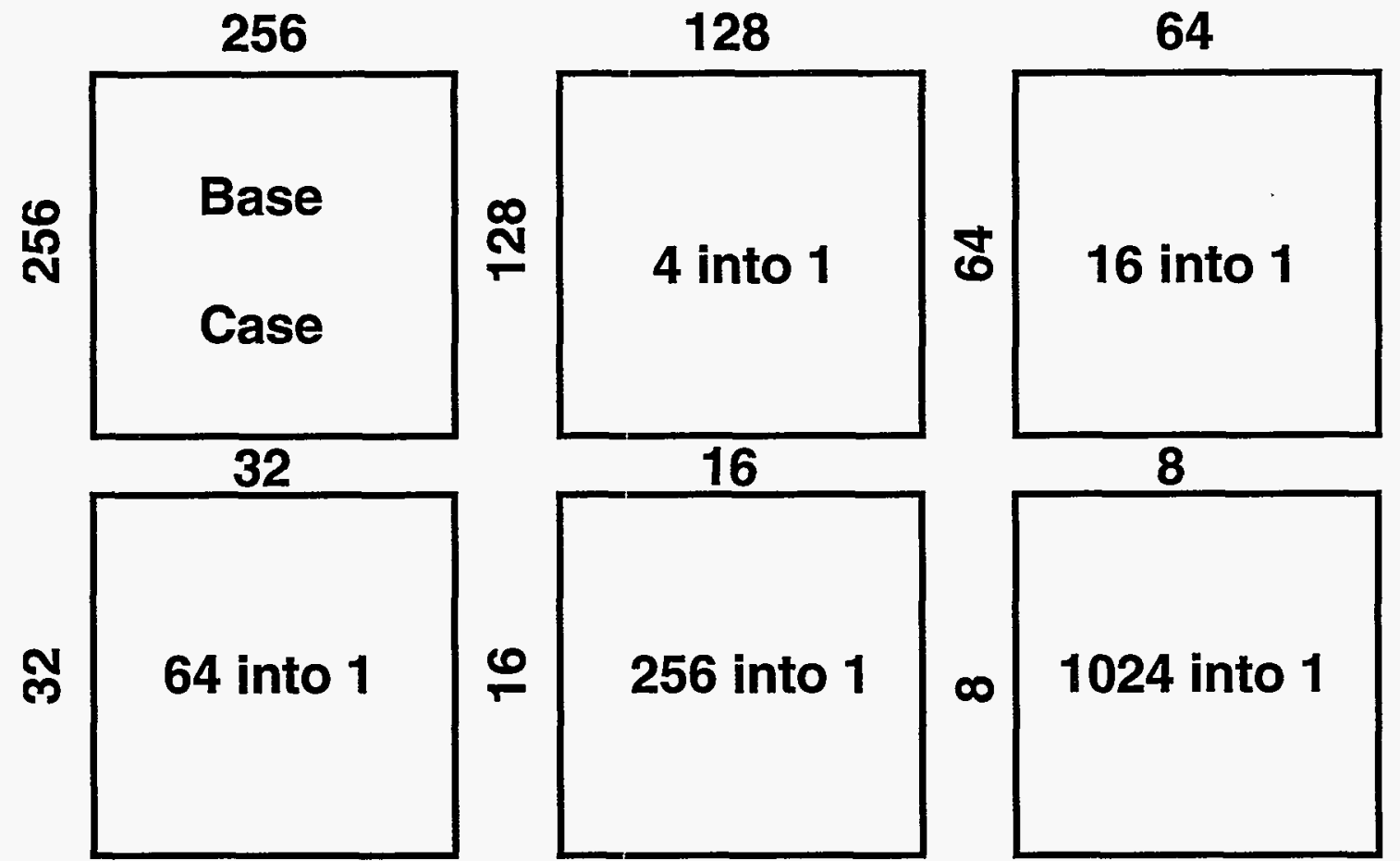

Figure 13. Conceptual representation of the five degrees of scaling examined by numerical experiments. The degree of scaling can be described as the number of calculational elements discretizing each side of the domain (e.g., 16x16) or by the number of point-scale values that constitute each upscaled element (256 into 1).

\section{Effect of Upscaling on Variance}

The principal effect of scaling is the loss of information from the point scale to the coarser scale. One measure of the quantity of information lost is the progressive decrease in variability of the log-conductivity field with continued upscaling. The loss of information with progressive upscaling for each of the seven scaling methods is presented graphically as the change in standard deviation of the upscaled field in figures 15,16 , and 17 .

The general effect of increasing the range of spatial correlation for the underlying pointscale field is to reduce the rate at which the standard deviation decreases with progressive upscaling. Both the absolute variability and the rate at which that variability diminishes with upscaling are similar for all scaling techniques except the renormalization algorithm and the spatial averaging technique. These two methods overestimate the standard deviation of the scaled fields relative 

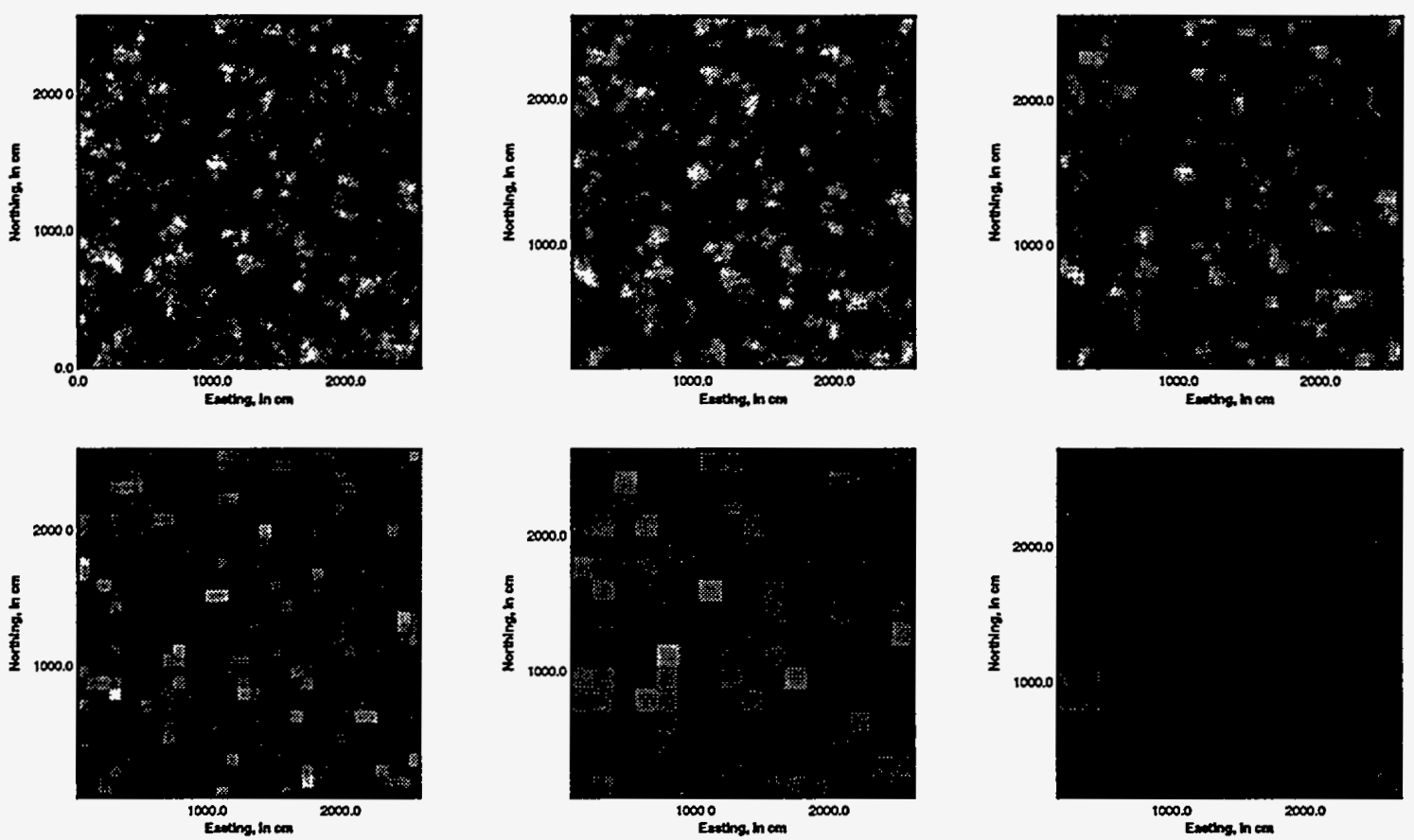

Figure 14. A point-scale field conductivity field with a range of $160 \mathrm{~cm}$ scaled to the five progressive levels of scaling using the geometric mean as the scaling algorithm.

to the other techniques. The rate at which the standard deviation, as determined by renormalization and spatial averaging, changes is also somewhat more erratic. Use of the geometric mean to scale the fields produces the lowest values of standard deviation across the different scales for all ranges of spatial correlation examined (more information is lost using this scaling technique). As the range of correlation $(\lambda)$ increases relative to the size of the upscaled element $\left(L_{e}\right)$, the different scaling techniques produce standard-deviation values that converge on a common value.

\section{Effect of Variability and Correlation Length on Upscaling of Isotropic Fields}

The effects of changes in both the range of spatial correlation and the degree of upscaling on the resulting effective conductivity values computed by the alternative scaling methods can be examined simultaneously by examining cases for which ratio of flow-element length to correlation length $\left(L_{e} / \lambda\right)$ is held constant. All cases for which $L_{e} / \lambda$ equals a constant have been subjected to effectively the same degree of upscaling. Table 5 is a matrix showing all possible combinations of $L_{e} / \lambda$ for the simulated point-scale log-conductivity fields. Note that the diagonals of the matrix in table 5 contain equal values of the $L_{e} / \lambda$ ratio. Upscaling the contained point-scale properties 

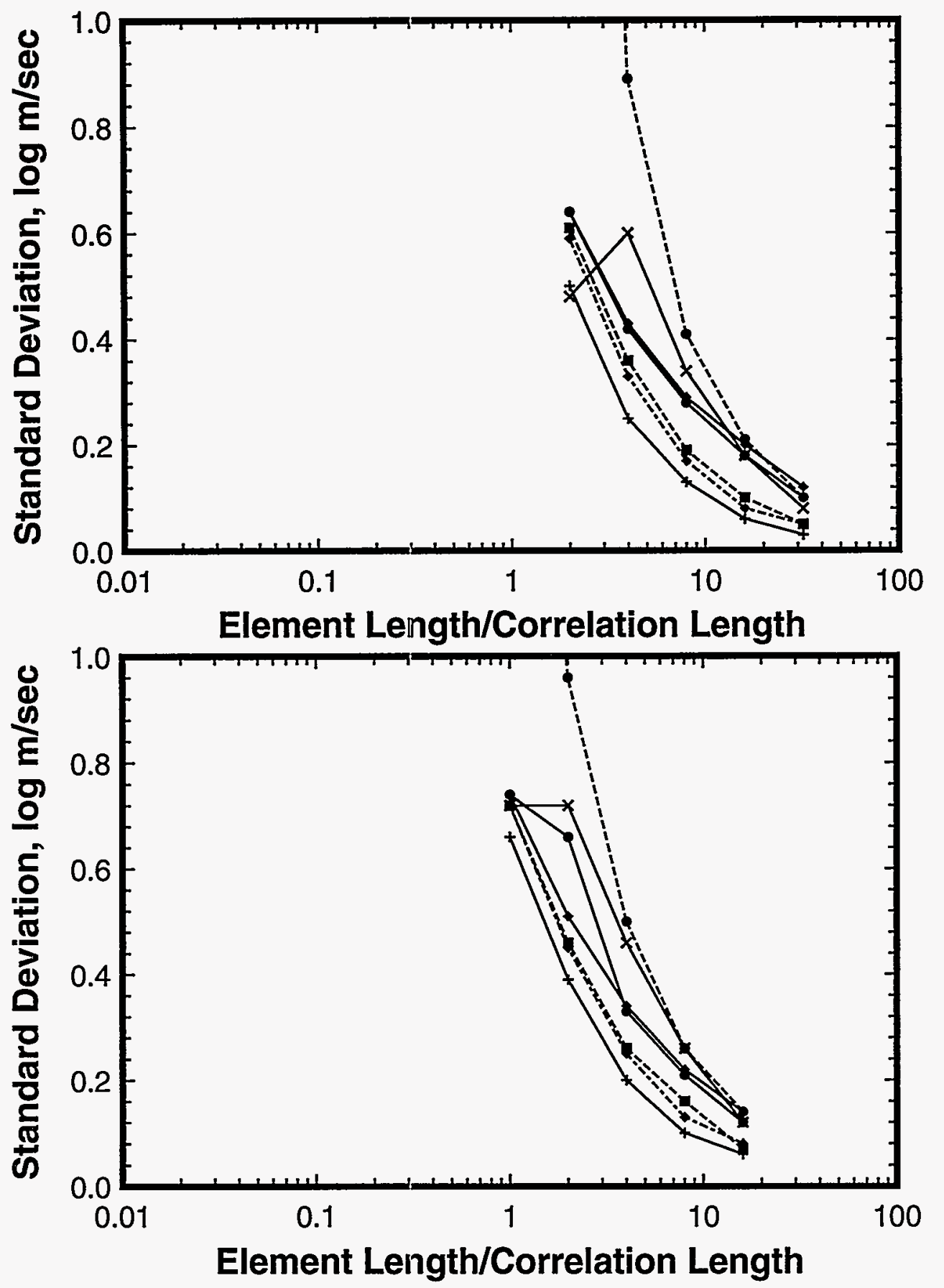

B

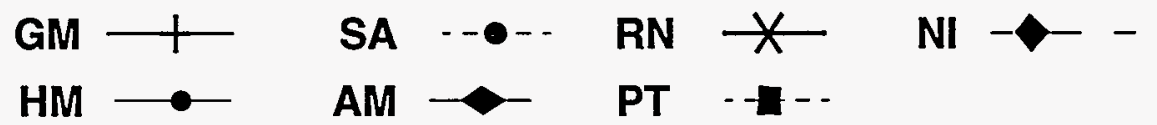

Figure 15. Graphs showing the standard deviation of $\log$ hydraulic conductivity as a function of the degree of upscaling: (A) $\lambda=10 ;(B) ; \lambda=20$. 

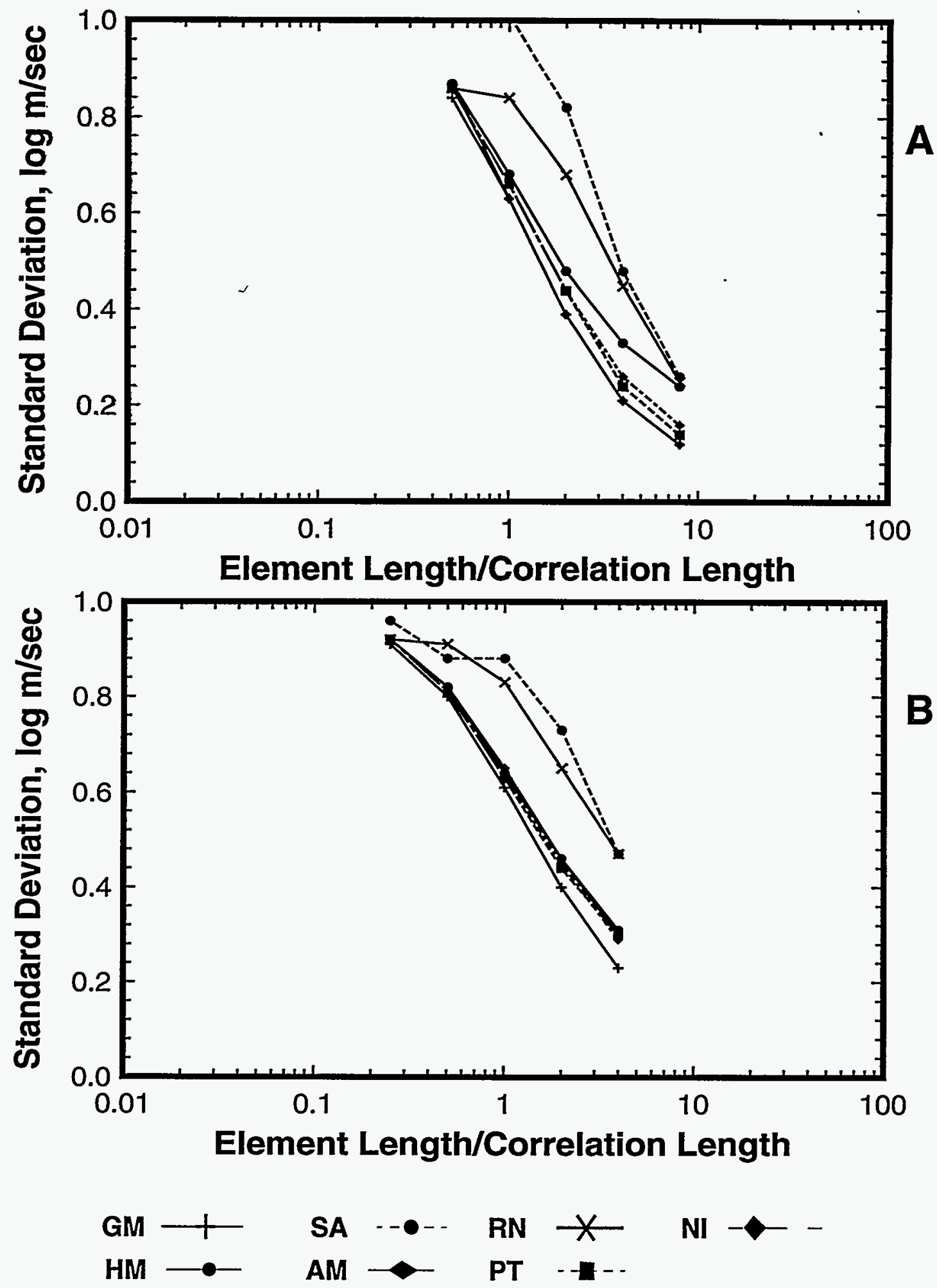

Figure 16. Graphs showing the standard deviation of $\log$ hydraulic conductivity as a function of the degree of upscaling: (A) $\lambda=40 ;(B) ; \lambda=80$. 


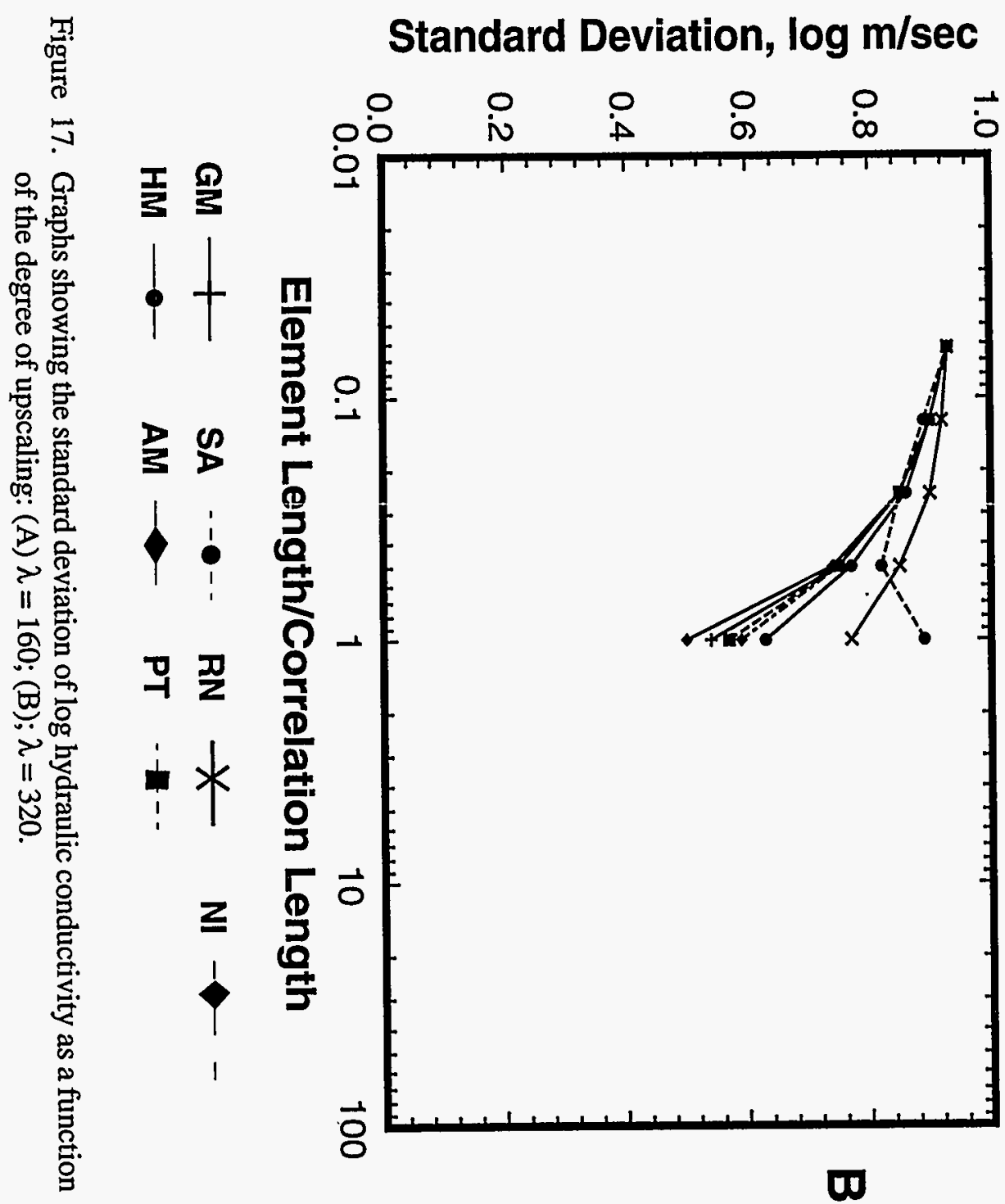

Standard Deviation, $\log \mathrm{m} / \mathrm{sec}$

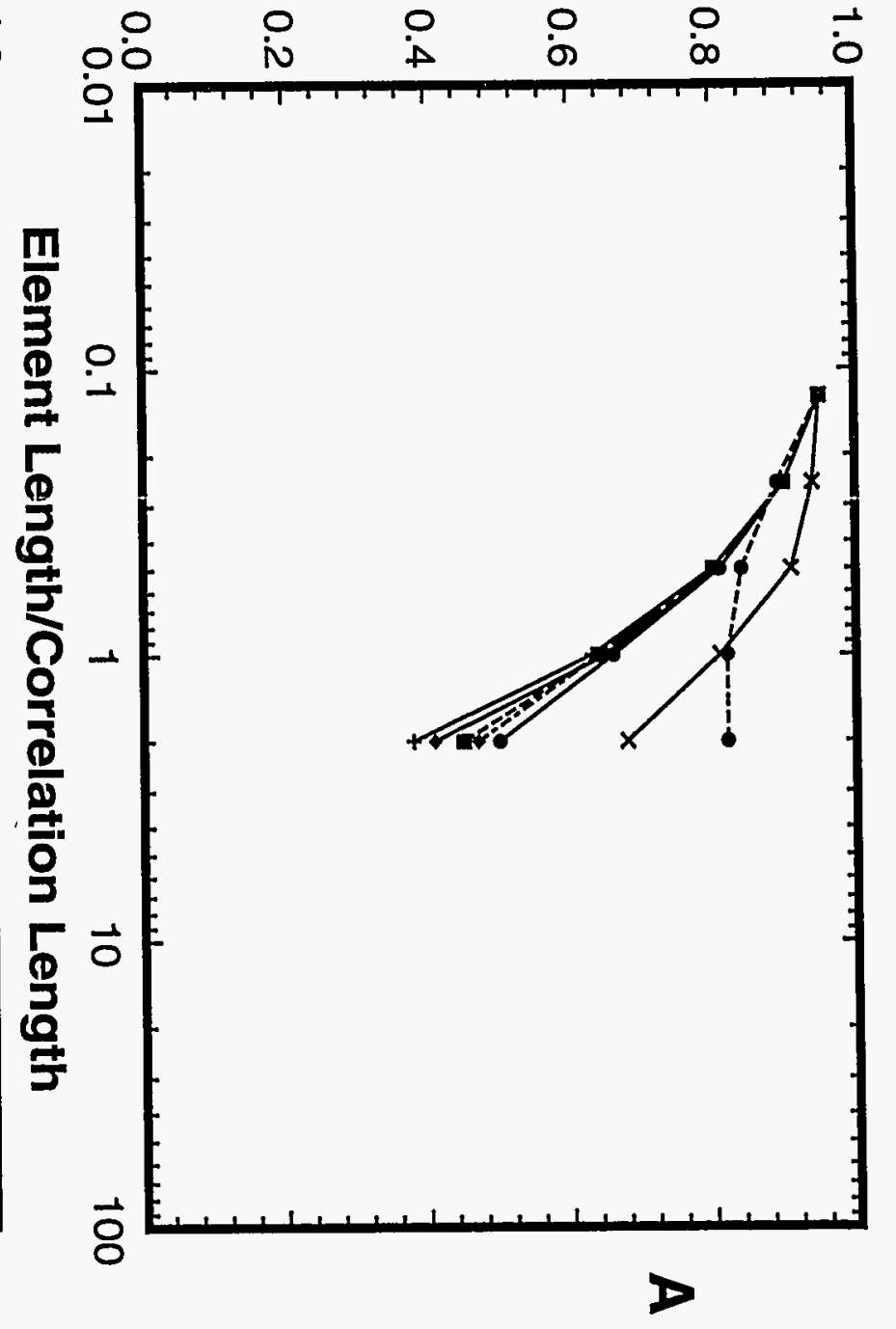


using a specific scaling algorithm for all $L_{e} \lambda \lambda$ combinations along a diagonal and solving the flow model for each combination provides an average response for a given degree of upscaling.

\begin{tabular}{|c|c|c|c|c|c|c|}
\hline \multirow{2}{*}{$\begin{array}{l}\text { Correlation } \\
\text { Length } \\
\text { (cm) }\end{array}$} & \multicolumn{6}{|c|}{ Flow-Element Length (cm) } \\
\hline & 10 & 20 & 40 & 80 & 160 & 320 \\
\hline 10 & 1 & 2 & 4 & 8 & 16 & 32 \\
\hline 20 & 0.5 & 1 & 2 & 4 & 8 & 16 \\
\hline 40 & 0.25 & 0.5 & 1 & 2 & 4 & 8 \\
\hline 80 & 0.125 & 0.25 & 0.5 & 1 & 2 & 4 \\
\hline 160 & 0.0625 & 0.125 & 0.25 & 0.5 & 1 & 2 \\
\hline 320 & 0.03125 & 0.0625 & 0.125 & 0.25 & 0.5 & 1 \\
\hline
\end{tabular}

For example, table 5 indicates six cases for which the flow-element length is equal to the spatial correlation length, or a $L_{e} / \lambda$ ratio, is equal to 1.0 (the middle diagonal). The hydraulic conductivity of the entire $2560-\mathrm{cm}$ by $2560-\mathrm{cm}$ flow model can be calculated for flow through each of these scaled conductivity fields. This effective hydraulic conductivity is then compared to the true hydraulic conductivity of the flow model, as calculated directly from the full $256 \times 256$ field of simulated point-scale conductivities (table 4). The performance measure for this evaluation of the scaling algorithms is defined as the percent difference between the computed effective conductivity value and the overall, point-scale conductivity. In the following figures, the results of the flow and transport calculations done on the isotropic point scale fields, shown in table 4, are the zero percent error case. Percent error is calculated as the values of the flow or transport parameter determined from the upscaled field minus the actual point scale value. This difference is divided by the point-scale value and the result is multiplied by 100 . This formulation causes underestimates of the point-scale value to have a negative percent error and overestimates to have a positive percent error. 


\section{Flow Properties}

The results of running the MODFLOW models using the upscaled, effective hydraulic conductivity values for each calculational element are shown in figure 18 in which the point-scale field results are represented by the zero error line. Generally, the effect of increasing the variability of the simulated $\log$ hydraulic-conductivity fields (the set simulated with $\sigma=1.0$; figure 18(b)) is to accentuate the trends observed for the conductivity fields simulated with the smaller level of variability $(\sigma=0.5$; figure $18(a))$.

The straightforward geometric mean, the numerical-inverse method, and the renormalization scaling techniques appear to perform relatively well across all levels of scaling. The percent error associated with flow modeled using computational elements scaled by these three methods are all less than approximately 5 percent. Conversely, the perturbation solution presented by Le Loc'h (1989) (equation 17) appears not to be an effective technique for upscaling to reproduce bulk hydraulic conductivity in isotropic fields. The spatial-averaging technique for upscaling appears to encounter difficulties when the local variance of the point-scale values contained within the boundaries of the upscaled flow elements is large. A large local variance occurs when the correlation length of the point-scale conductivity field is approximately equal to the length of the upscaled flow element (an $L_{e} / \lambda$ near 1 ), and there is a relatively small number of point values within the confines of that element.

\section{Transport properties}

The results for transport properties computed using the scaled hydraulic conductivity fields and the MODFLow flow model are presented graphically in figures 19 through 22 . As expected, those scaling techniques that best reproduce the bulk hydraulic conductivity of the flow field also reproduce, though to a somewhat lesser extent, critical transport properties as indicated by the particle-tracking results. Those scaling methods that performed poorly in reproducing the large-scale conductivity also performed poorly in modeling transport. 

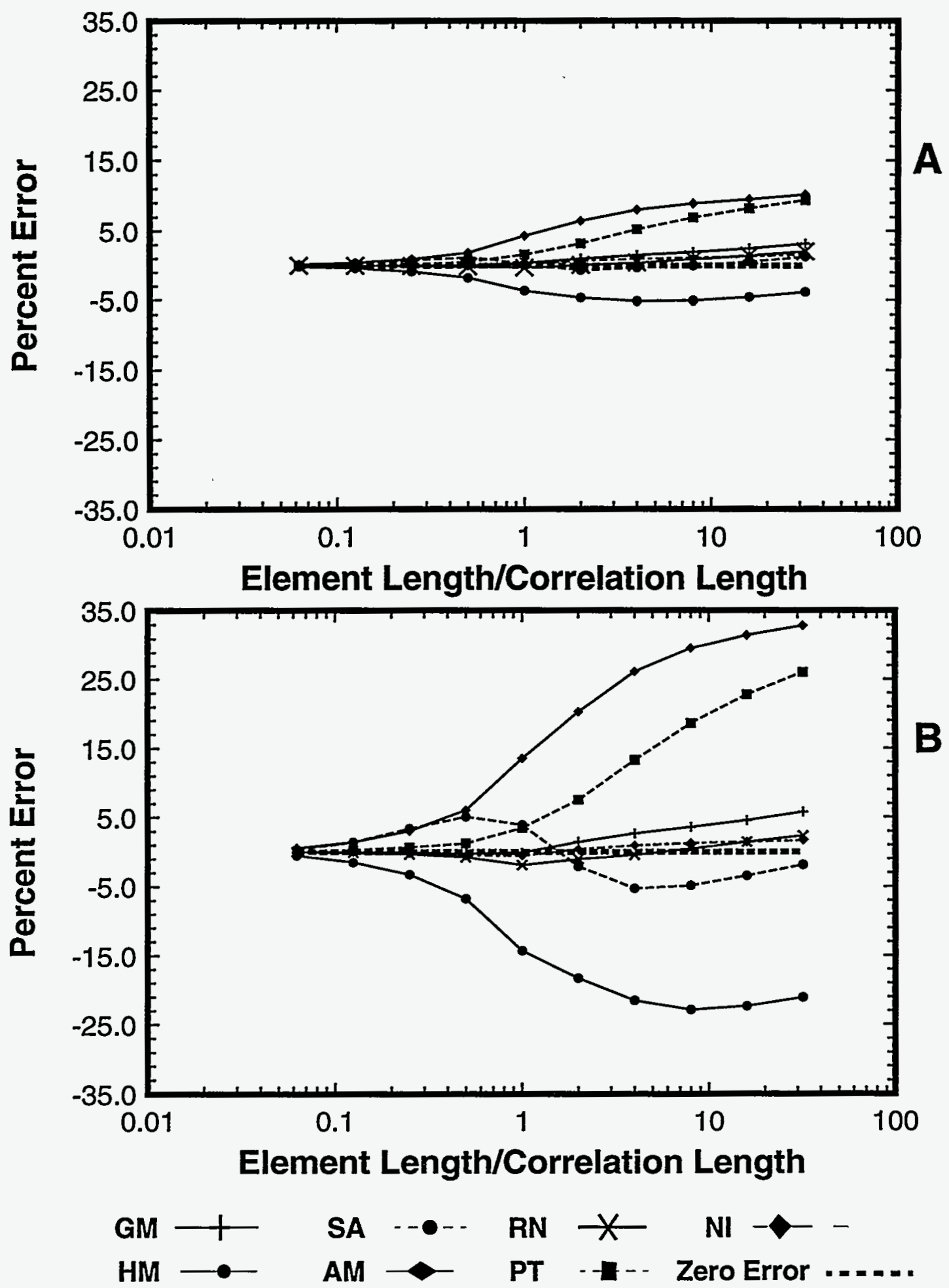

Figure 18. Graph showing percent error in the computed hydraulic conductivity of the 2560$\mathrm{cm}$ by $2560-\mathrm{cm}$ flow model as a function of grid-element length to correlation length ratio $\left(L_{e} / \lambda\right)$. Standard deviation of underlying point-scale conductivity simulations equal to (A) 0.5 ; (B) 1.0 . 
Results for the mean particle travel time through the entire flow-model domain are shown in figure 19. Because the ultimate application of this scaling work will be in performance assessment calculations, one of which is the estimation of the fastest ground-water travel time, it is important to evaluate the impacts of different scaling techniques on the fastest particle velocity at different levels of upscaling. The results for the fastest particle velocity presented in figure 20 .
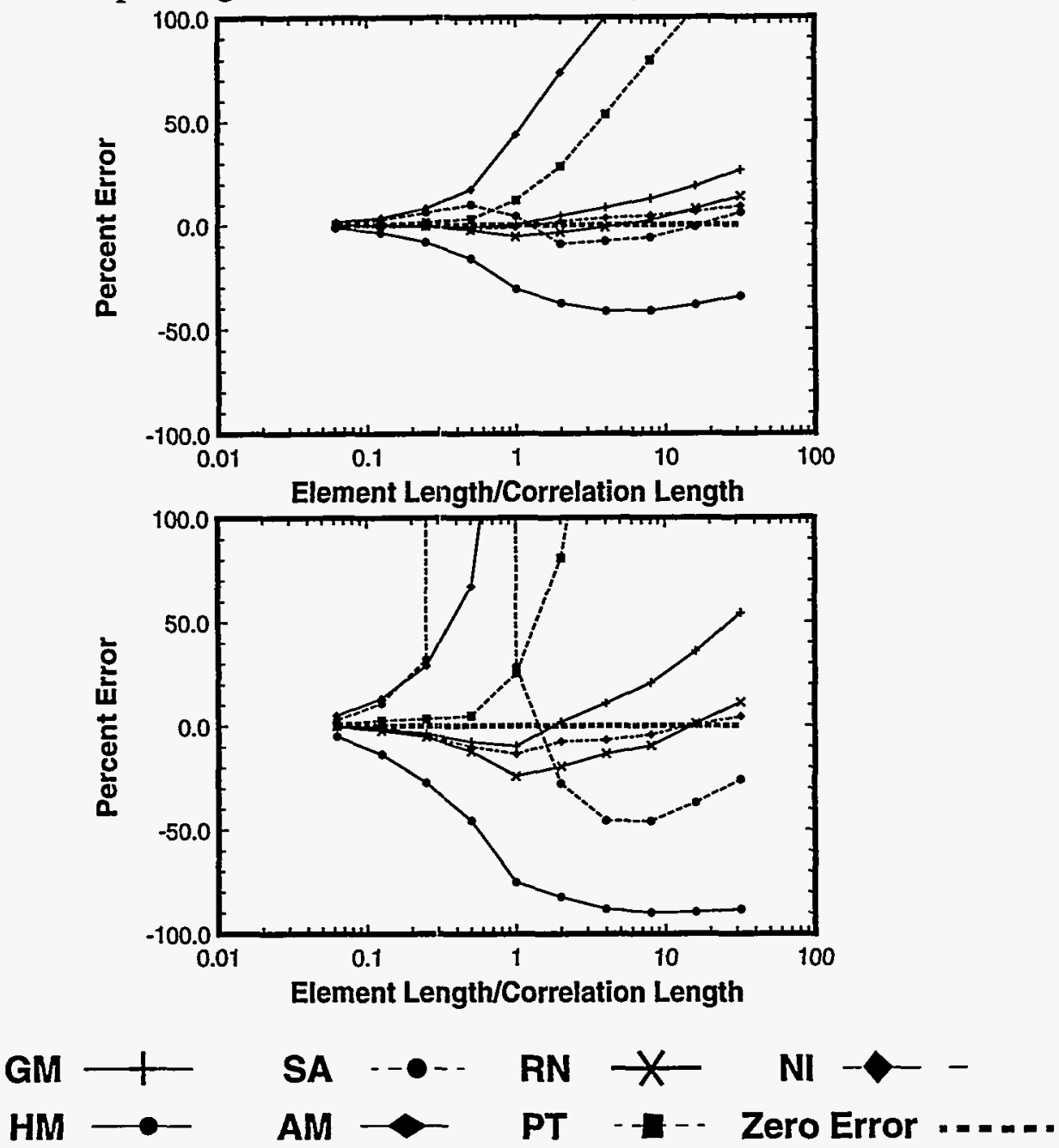

Figure 19. Graph showing percent error in mean particle velocity through the $2560-\mathrm{cm}$ by $2560-\mathrm{cm}$ flow model as a function of grid-element length to correlation length ratio $\left(L_{e} / \lambda\right)$. Standard deviation of underlying point-scale conductivity simulations equal to (a) 0.5 ; (b) 1.0 .

Essentially all scaling techniques provide adequate reproduction of both the mean particle velocity and the fastest particle velocity when the flow-element length to spatial-correlation length ratio is small $(L / \lambda$ less than 0.1$)$. As this ratio increases, the percent error for both the mean 

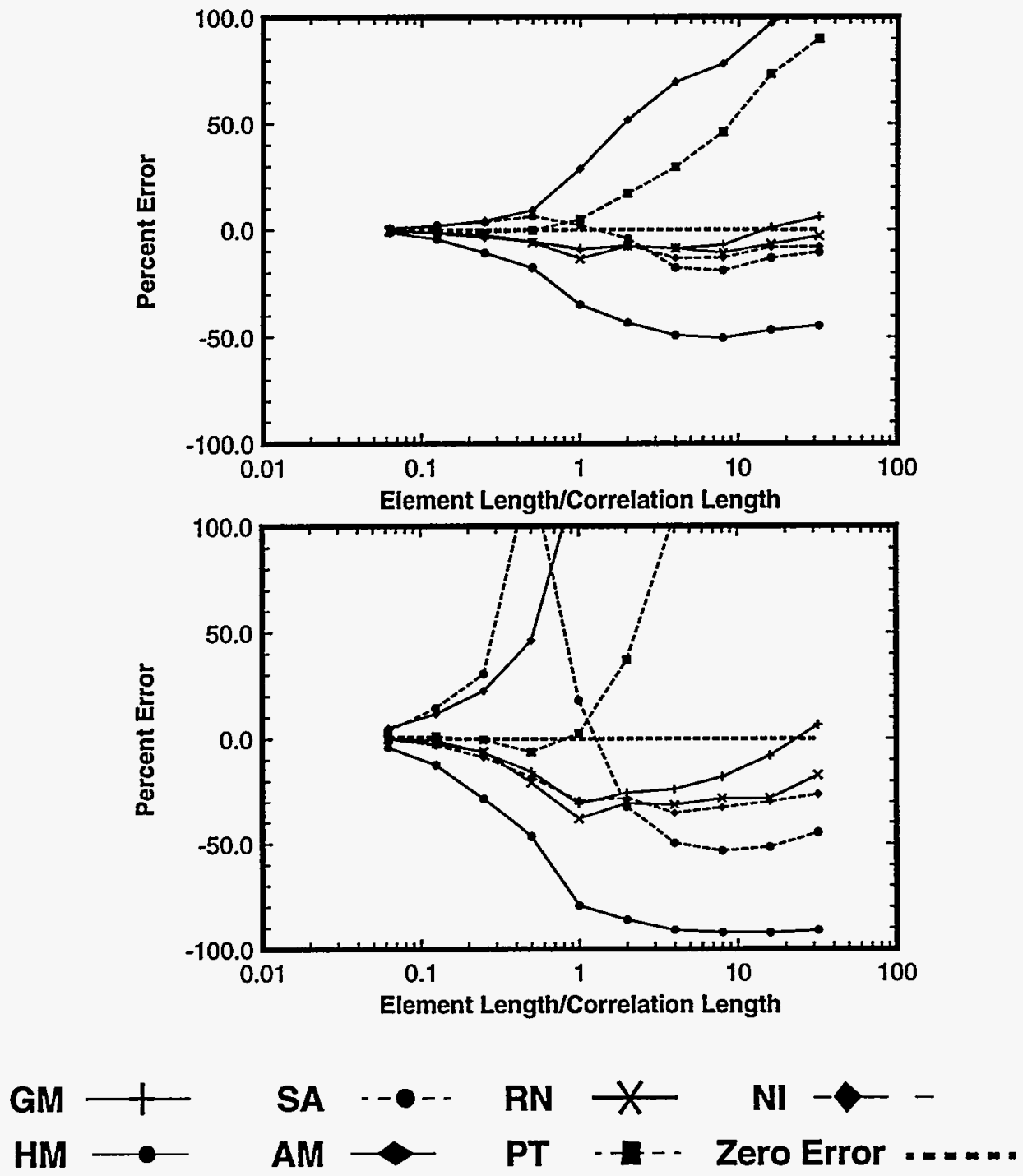

Figure 20. Graph showing percent error in fastest particle velocity through the $2560-\mathrm{cm}$ by $2560-\mathrm{cm}$ flow model as a function of grid-element length to correlation length ratio $\left(L_{e} / \lambda\right)$. Standard deviation of underlying point-scale conductivity simulations equal to (a) 0.5 ; (b) 1.0 .

and fastest particle velocities increases. Note that although the geometric mean, the numerical inverse, and the renormalization scaling methods provide the best reproduction of the actual mean and fastest travel times, the mean travel times are underestimated at low $L_{e} / \lambda$ ratios and overestimated at higher ratios. The fastest particle velocity is consistently underestimated by the same three techniques. The arithmetic mean and the spatial-averaging techniques provide completely unrealistic results for the more variable point-scale simulations $(\sigma=1.0)$. 
The influence of the different scaling methods and the degree of scaling on dispersion was examined by calculating the dispersivity $\left(\alpha_{L}\right)$ and longitudinal dispersion coefficients $\left(D_{l}\right)$ for each upscaled flow model using the computed Peclet number (eq. 20) and equations 21 and 22. The reproduction of these two transport parameters for the upscaled conductivity fields are compared with the equivalent point-scale parameters in figures 21 and 22 . Note that although all scal-
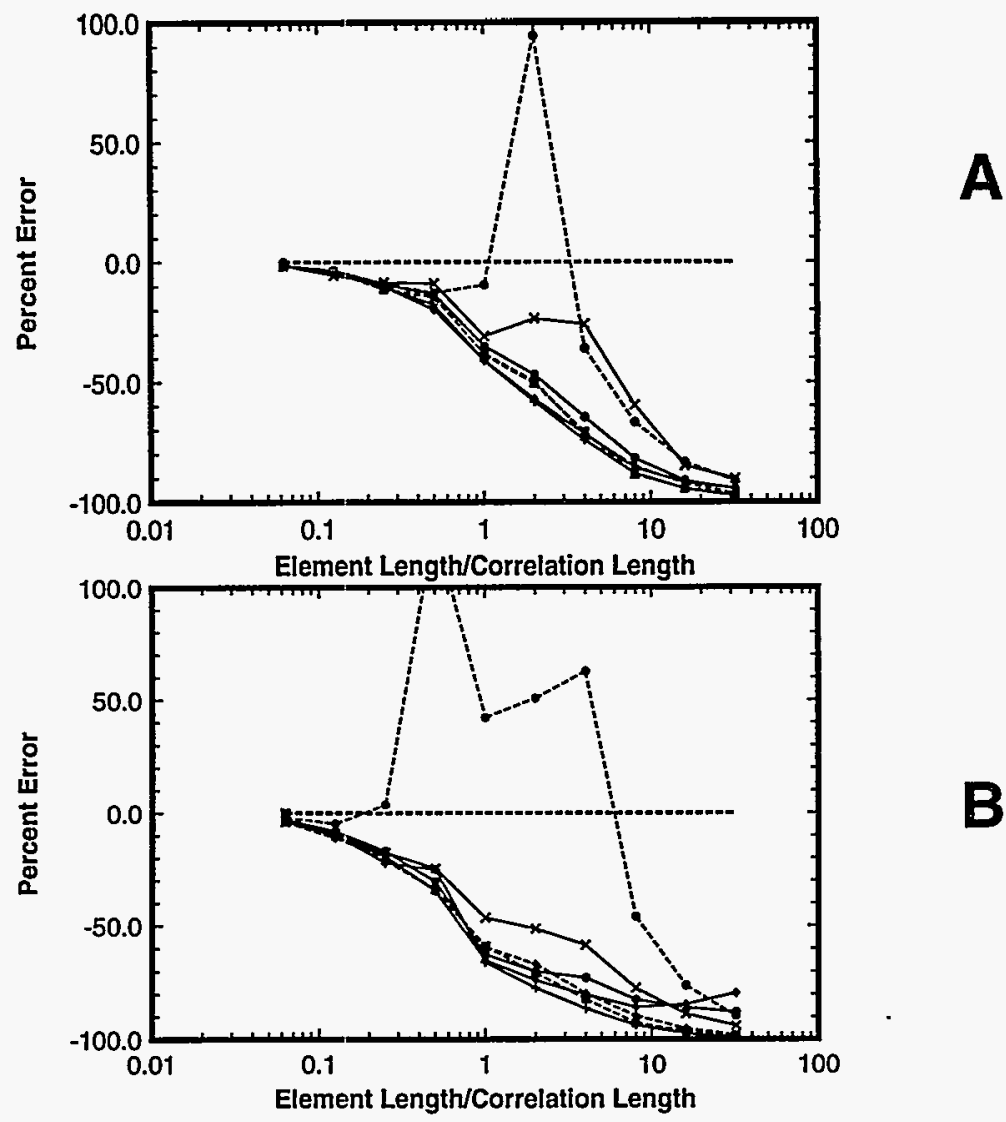

B

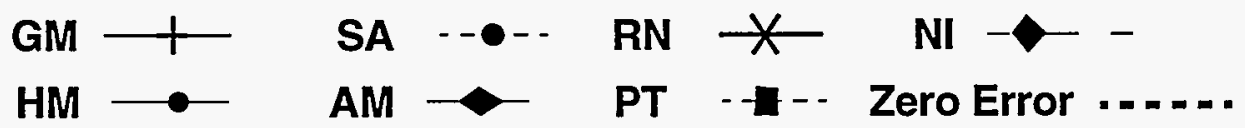

Figure 21. Graph showing percent error in longitudinal dispersivity through the 2560 -cm by $2560-\mathrm{cm}$ flow model as a function of grid-element length to correlation length ratio $\left(L_{e} / \lambda\right)$. Standard deviation of underlying point-scale conductivity simulations equal to (A) 0.5 ; (B) 1.0 .

ing methods except the spatial averaging technique provide consistent dispersion results, none of those methods provides a particularly accurate reproduction of the calculated point-scale dispersivity when the $L_{e} / \lambda$ ratio exceeds 0.1 (figure 21 ). Reproduction of the dispersion coefficient is 


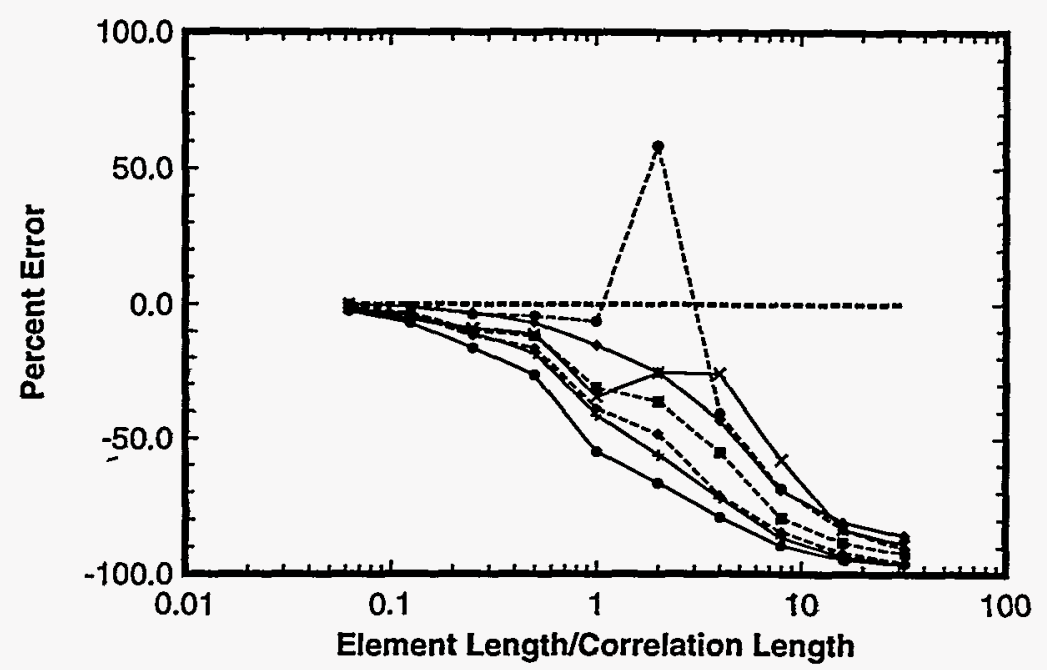

A

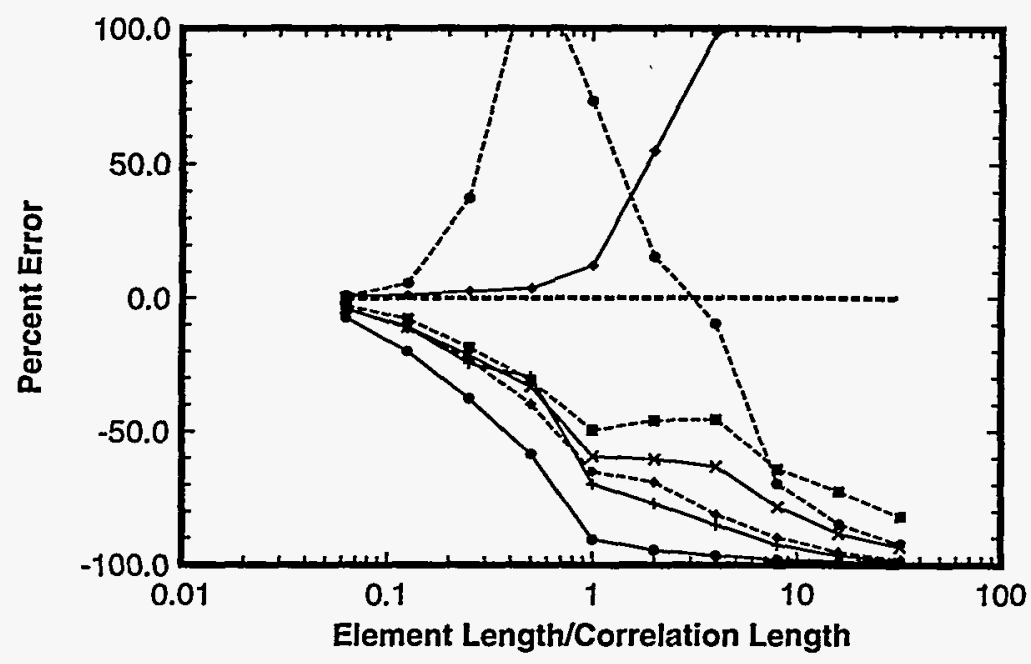

B

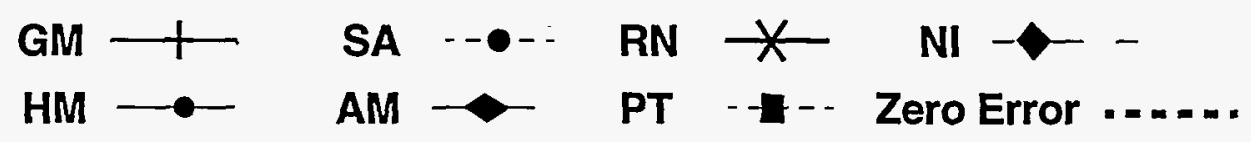

Figure 22. Graph showing percent error in longitudinal dispersion coefficient as a function of grid-element length to correlation length ratio $\left(L_{e} / \lambda\right)$. Standard deviation of underlying point-scale conductivity simulations equal to (A) 0.5 ; (B) 1.0 .

reasonable up to $L_{e} \lambda \lambda$ ratios of slightly less than one for the upscaled, less-variable simulated conductivity fields, for which the standard deviation of conductivity values is 0.5 [figure 22(a)]. For the upscaled, more-heterogeneous $(\sigma=1.0)$ simulated fields, the reproduction of the dispersion coefficient is similar to the patterns observed for dispersivity. Interestingly, the arithmetic mean provides the best estimate of the modeled, true dispersion coefficient for flow-element sizes up to the range of spatial correlation for both data sets. 
The fastest particle velocity is underestimated by a number of techniques and this result has implications for the GWTT 95 study. This underestimation of fastest particle velocity is attributed to the fastest flowpaths in the point scale fields being smoothed away in the scaling process. In a groundwater travel time calculation, the effects of this smoothing are counteracted by adding dispersion to the particles. Because both longitudinal dispersivity and the dispersion coefficient have been calculated in this study, it is possible to determine the dispersivity value required to keep the dispersion coefficient equal to that observed for the point-scale grid at larger degrees of upscaling. The amount of dispersion occurring in the cloud of tracked particles in these numerical experiments is caused only by changes in hydraulic conductivity between flow-model grid elements. There is no molecular diffusion to contribute to hydrodynamic dispersion, and mechanical dispersion is related only to variations in hydraulic conductivity. Mixing caused by variations in pore-space geometry and connectivity has not been modeled. Therefore, we can write:

$$
\text { Modeled Dispersion is } \mathrm{D}_{m}=\alpha_{L} V_{x} \text {, }
$$

where $\alpha_{L}$ is the longitudinal dispersivity, and $V_{x}$ is the mean particle velocity.

The scale of the flow-model grid element is the smallest scale of heterogeneity that can be represented in the numerical flow model. The dispersion in the flow direction (longitudinal) has been tabulated at each level of upscaling. By adjusting the longitudinal dispersivity value $\left(\alpha_{\mathcal{L}}\right)$ in eq. 24, the amount of modeled dispersion can be kept constant across all levels of scaling. The dispersivity values required to maintain constant dispersion with progressive upscaling are shown in Figure 23.

The relationships presented in Figure 23 can be used to determine the dispersivity value that will reproduce the amount of dispersion modeled at the point scale for any arbitrary level of upscaling. From Figure 23, it appears that this relationship is essentially piece-wise linear. One linear relationship describes the appropriate dispersivity value at $L_{e} / \lambda$ ratios below 1.0 , and a sec- 

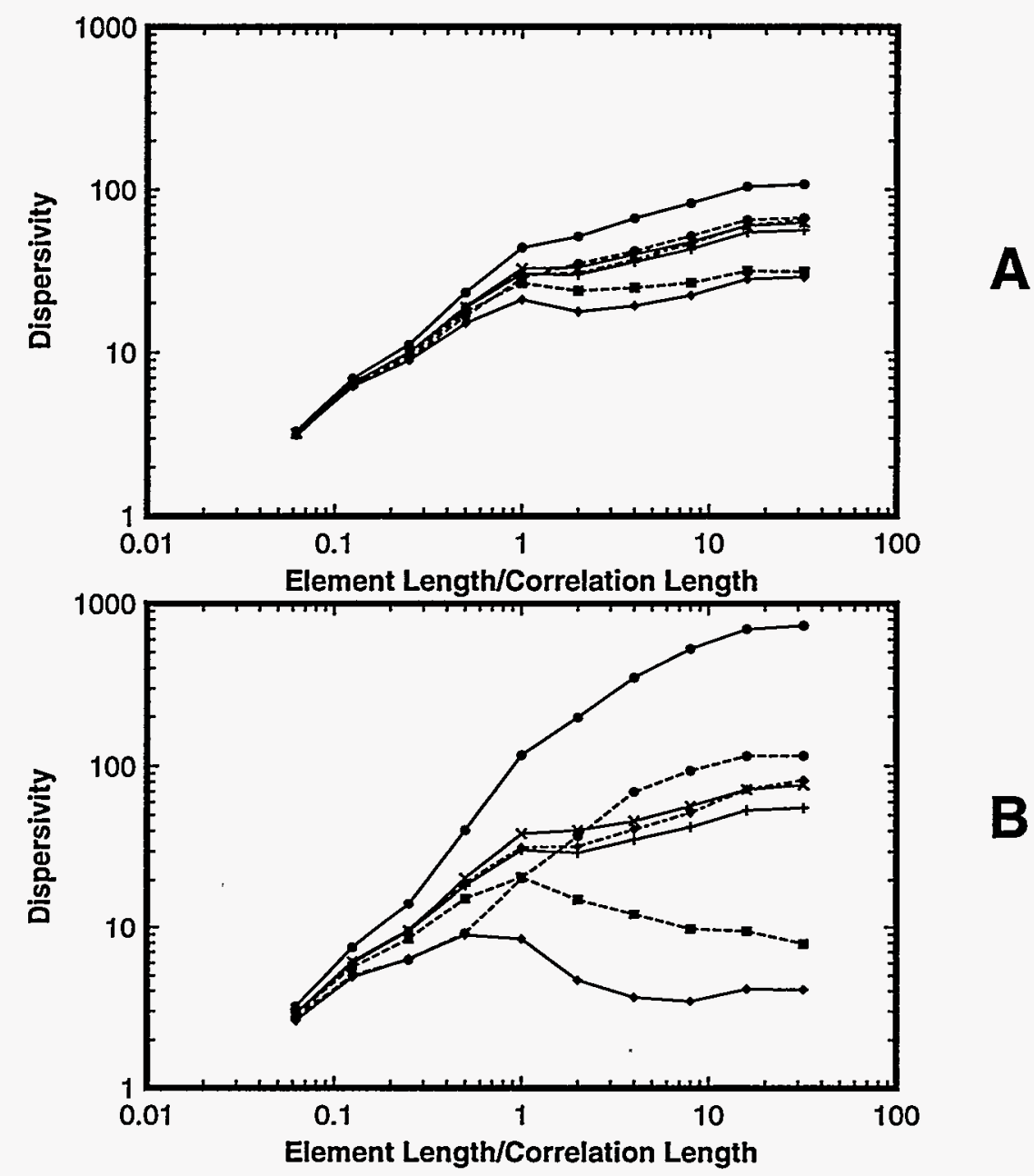

B

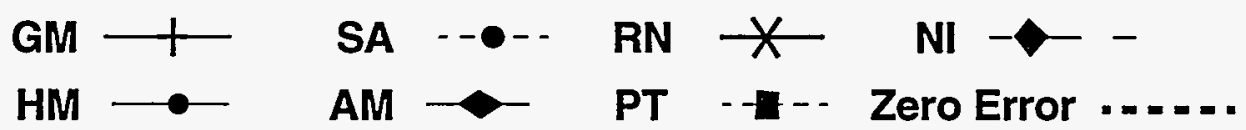

Figure 23. Graph showing dispersivity $\left(\alpha_{1}\right)$ values that will reproduce the amount of pointscale dispersion as a function of grid-element length to correlation length ratio $\left(L_{e} / \lambda\right)$. Standard deviation of underlying point-scale conductivity simulations equal to (A) 0.5 ; (B) 1.0 .

ond relationship describes this value at ratios above 1.0. In case of the more heterogeneous simulated field $(\sigma=1)$, it appears that the required dispersivity is approaching a constant value as the flow-element length to spatial-correlation length ratio, $L_{e} / \lambda$, increases. This asymptotic behavior is consistent with numerical and analytical results obtained by Dykaar and Kitanidis (1992b), who showed that an $L_{e} / \lambda$ ratio of 80 was necessary to assign an effective property value to an upscaled block. 
The piece-wise linear relationship of dispersivity as a function of scaling was determined by fitting the equation of a straight line to the two portions of each graph. To achieve the most statistical mass for the regressions, data for the three scaling techniques that gave the lowest percent error for mean particle travel time (geometric mean, numerical inverse, and renormalization) were aggregated prior to curve-fitting.

For the case in which the standard deviation of the point-scale simulated conductivities was equal to 0.5 , the two equations are:

$$
\begin{array}{llll}
\text { Dispersivity }=29.1\left(L_{e} / \lambda\right)+2.6 & \text { for } & L_{e} / \lambda \leq 1, \text { and } & \text { eq. } 25 \\
\text { Dispersivity }=1.0\left(L_{e} / \lambda\right)+33.3 & \text { for } & L_{e} / \lambda>1 . & \text { eq. } 26
\end{array}
$$

For the more variable case, for which the standard deviation of the underlying, point-scale simulated field is equal to 1.0 , the equations are:

$$
\begin{aligned}
& \text { Dispersivity }=32.6\left(L_{e} / \lambda\right)+1.7 \text { for } \quad L_{e} / \lambda \leq 1 \text {, and } \quad \text { eq. } 27 \\
& \text { Dispersivity }=1.3\left(L_{e} / \lambda\right)+35.9 \text { for } \quad L_{e} / \lambda>1 \text { eq. } 28
\end{aligned}
$$

Equations 25 through 28 have been developed with assumed length units of centimeters. However, the relationships should hold for any length units as $L_{e} / \lambda$ is dimensionless if the flowmodel element size and the spatial-correlation length are both specified in the same units and in the same units as dispersivity.

\section{Flow-and-Transport Results for Anisotropic Conductivity Profile}

Results of using power-law averaging on the simulated, anisotropic hydraulic conductivity field of figure 8 are presented in a manner similar to that used to evaluate the results of scaling for the isotropic conductivity fields. Figures show the power-law averaging results in terms of percent error plotted against the relevant power-law coefficient. The various levels of scaling are shown 
by the different line and symbol styles in the figures. The objective is to replicate the point-scale results that are summarized as the zero-error line.

\section{Flow Properties}

The results of the MODFLOW calcualtions with fixed heads on the ends, no flow boundaries on the sides, and using the upscaled conductivity values for each calculational element are presented in table 6 . The results in table 6 show the value of the power coefficient that gave the low-

Table 6: Flow-and-Transport Results Using Power-Law Averaging to Create Upscaled Flow-Model Hydraulic Conductivity Values from Simulated Point-Scale Conductivities

\begin{tabular}{|c|c|c|c|c|c|c|c|c|c|c|}
\hline \multirow{3}{*}{ Parameter } & \multicolumn{10}{|c|}{ Level of Upscaling } \\
\hline & \multicolumn{2}{|c|}{ None } & \multicolumn{2}{|c|}{$4: 1$} & \multicolumn{2}{|c|}{$16: 1$} & \multicolumn{2}{|c|}{$64: 1$} & \multicolumn{2}{|c|}{$256: 1$} \\
\hline & Power & Value & Power & $\begin{array}{c}\% \\
\text { Error }\end{array}$ & Power & $\begin{array}{c}\% \\
\text { Error }\end{array}$ & Power & $\begin{array}{c}\% \\
\text { Error }\end{array}$ & Power & $\begin{array}{c}\% \\
\text { Error }\end{array}$ \\
\hline Bulk K $(\mathrm{m} / \mathrm{sec})$ & NA & -11.05 & -0.5 & -0.01 & -0.5 & 0.09 & -0.6 & -0.12 & -0.6 & 0.01 \\
\hline (m/year) & $\mathrm{NA}$ & $8.8 e-6$ & -0.4 & -1.91 & -0.4 & -0.71 & -0.4 & -0.68 & -0.4 & -2.48 \\
\hline (m/year) & NA & $1.29 e-5$ & -0.3 & -1.47 & -0.3 & -1.94 & -0.2 & 7.14 & -0.2 & -2.56 \\
\hline $\begin{array}{l}\text { (a) } \\
\text { (m) }\end{array}$ & NA & 34.8 & 1.0 & -14.04 & 0.3 & -4.83 & 0.2 & 13.82 & 0.2 & 3.79 \\
\hline $\begin{array}{c}\text { Dispersion } \\
\text { Coeff. } \\
\left(\mathrm{D}_{1}\right) \\
\left(\mathrm{m}^{2} / \text { year }\right)\end{array}$ & NA & $3.1 \mathrm{e}-4$ & 0.1 & -4.35 & 0.0 & 3.43 & 0.0 & -1.78 & 0.0 & -14.89 \\
\hline
\end{tabular}

est percent error and that level of error. Negative errors represent an underestimation of the actual value and positive errors an overestimation. These results are presented graphically in figure 24, using the percent error of the scaled conductivity flow-model runs compared to the results from the full, point-scale, MODFLOW calculations as the performance measure.

A power coefficient $(w)$ of approximately -0.5 to -0.6 reproduces the overall hydraulic conductivity of the model computed from the point-scale material-property field for all levels of scaling. The numerical experiments involving the isotropic, point-scale fields suggested that the value of $w$ would be closer to zero, representing the geometric mean (compare to figure 18). This 

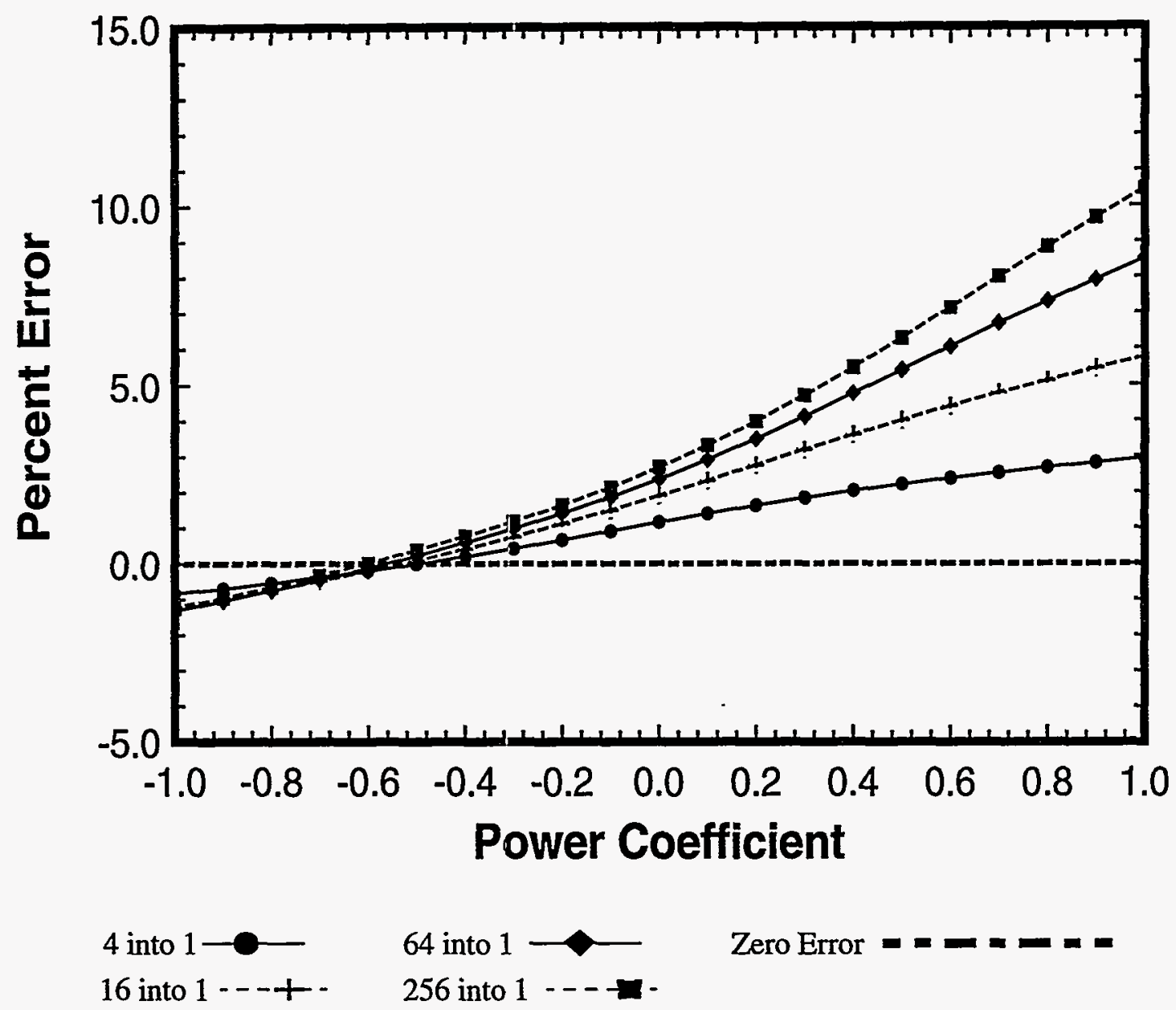

Figure 24. Graph showing percent error reproducing effective hydraulic conductivity of the simulated cross-sectional model of Yucca Mountain as a function of the powerlaw coefficient, $w$.

discrepancy follows logically from anisotropy of the hydraulic-conductivity field represented by the cross section of Yucca Mountain (figure 8).

Anisotropy at Yucca Mountain results from subhorizontal stratigraphic layering of the alternating welded and nonwelded tuffs. The presence of pronounced stratigraphic layering, plus definition of the boundary conditions for the numerical flow-and-transport model, creates a flow geometry similar to that of figure 1(a). The harmonic average, corresponding to a power-law coefficient of -1.0 , would reproduce the effective hydraulic conductivity in a perfectly layered system where the average gradient is normal to the layering. The observed value of $w=-0.5$ to -0.6 is thus consistent with flow through an imperfectly, but strongly layered system with flow occurring 
at an angle of somewhat less than $90^{\circ}$ to that layering, such as is represented by figure 8 . The error, in terms of reproducing the effective hydraulic conductivity of the entire flow system using geometric mean, is only about 2 percent (figure 24 ).

\section{Transport Properties}

The results of the MODFLOW calculations with respect to the transport properties for the cross-sectional model of Yucca Mountain are also given in table 6 and in figures 25 through 28 . Generally, a single power coefficient can be identified, at which all levels of upscaling essentially reproduce the transport property value of the detailed, point-scale flow model. However, this power coefficient is a function of the performance measure examined. The relative error of the scaled flow models increases progressively with greater levels of upscaling.

Reproduction of average particle travel time through the flow field appears to follow that of the effective, overall hydraulic conductivity of the cross section (figure 25). A value of $w=-$ 0.4 , produces essentially zero error across all levels of scaling. The best-fit value of $w$ for hydraulic conductivity is -0.5 to -0.6 . It appears that average particle travel time also reflects the combined influence of stratigraphic layering and the specified boundary conditions of the flow model. Scaling of the point-scale conductivity field using the geometric mean with $w=0$, produces a twenty to sixty-percent error in reproduction of the mean particle velocity.

Reproduction of the fastest particle travel time through the scaled hydraulic-conductivity cross section is closest for values of $w=-0.3$ to -0.2 (figure 26). The appropriate power coefficient increases from -0.3 to -0.2 as the degree of upscaling increases from four point-scale values into one grid cell (4:1) to $256: 1$. Computing the scaled conductivity values of each flow element using the geometric mean $(w=0)$ produces errors of 20 to 40 percent for the fastest travel time.

The dispersion behavior of the upscaled hydraulic-conductivity models of the Yucca Mountain cross section appears somewhat different from that of travel time, as computed using particle tracking. In contrast to the behavior represented in figures 24 through 26 , there appears to 


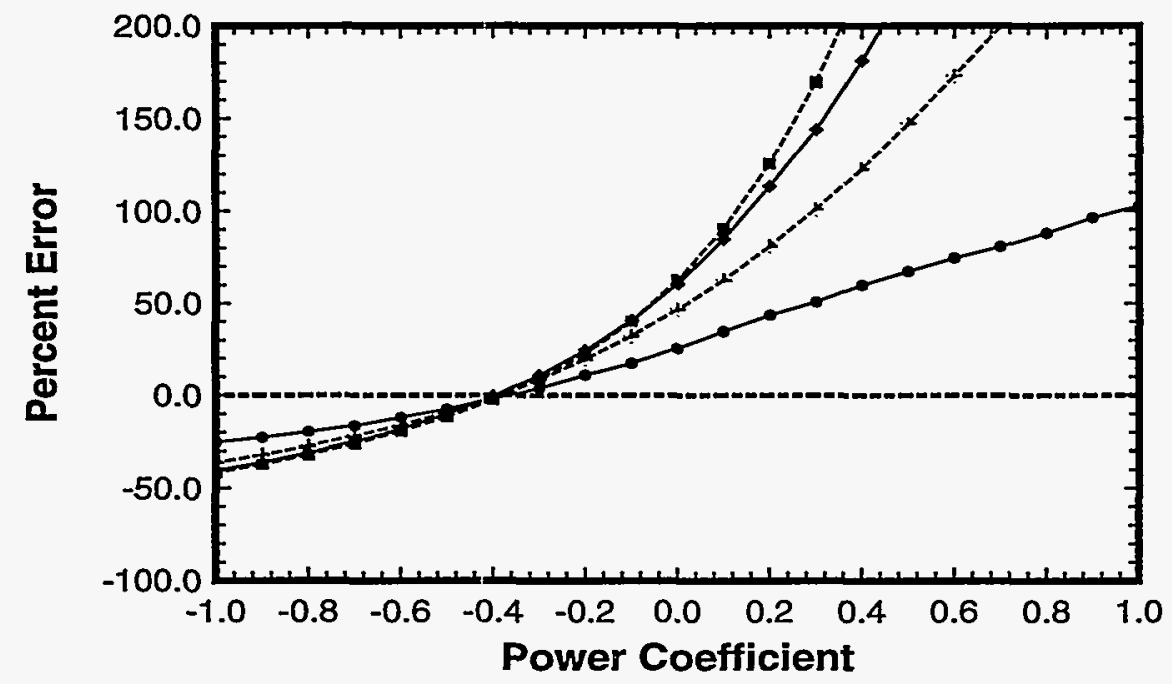

4 into $1-$

16 into 1 - - -

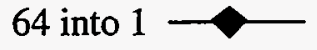

Zero Error

Figure 25. Graph showing percent error reproducing average travel time through the simulated cross-sectional model of Yucca Mountain as a function of the powerlaw coefficient, $w$.
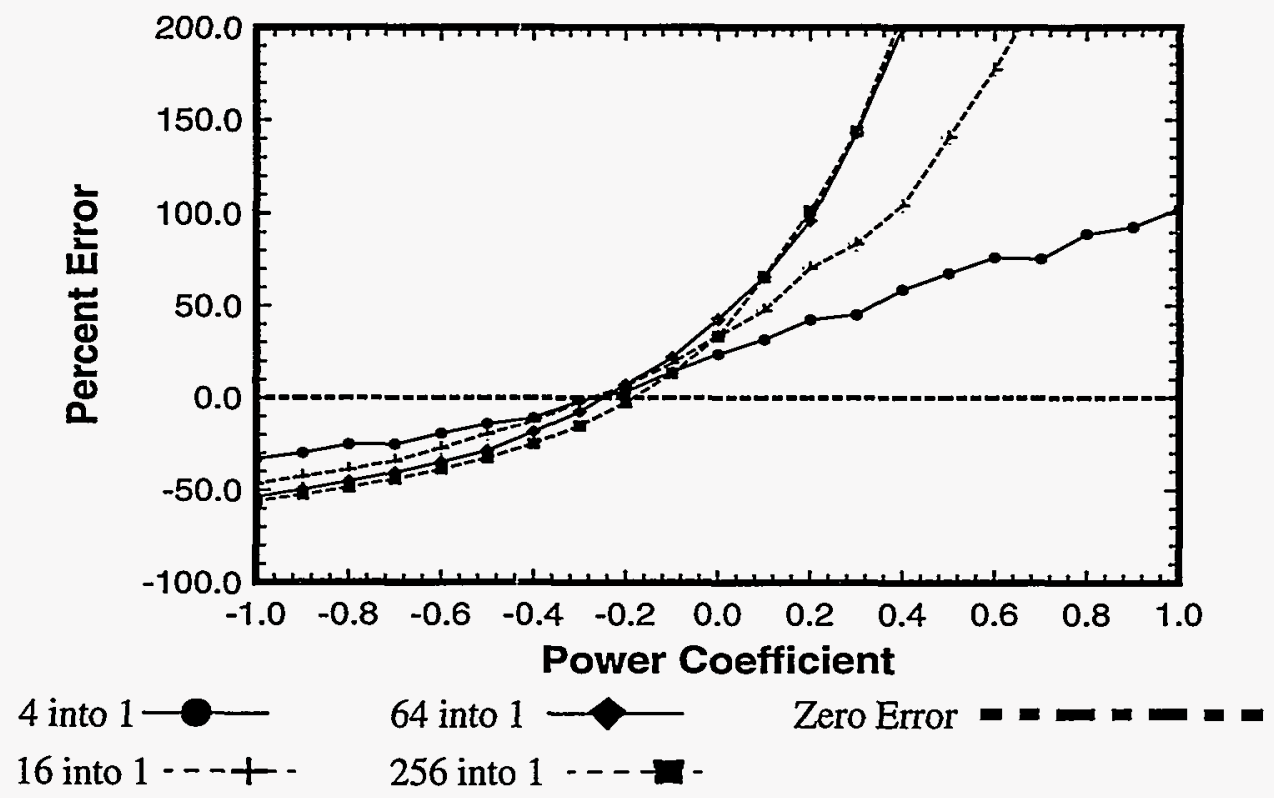

Figure 26. Graph showing percent error reproducing fastest travel time through the simulated cross-sectional model of Yucca Mountain as a function of the power-law coefficient, $w$.

be no single power coefficient at which the different levels of upscaling can be characterized. For example, the percent error in reproducing the point-scale longitudinal dispersivity by the upscaled flow models is shown in figure 27. At the lowest level of upscaling, averaging 4 point-scale con- 
ductivities into one flow model element, the longitudinal dispersivity is underestimated for all values of $w$. Dispersivity can be considered as the distance over which a given amount of mixing takes place between two distinct phases of a fluid. The 4:1 upscaling appears to predict that this distance is less than what is actually observed for the point-scale field. This anomalous effect appears to be caused by the first level of upscaling producing a markedly less-variable conductivity field than is actually present. At higher levels of scaling, notably for the $64: 1$ and $256: 1$ averaging grid cells, the value of $w$ that reproduces the point-scale dispersivity appears to converge on a single value of approximately 0.17 .

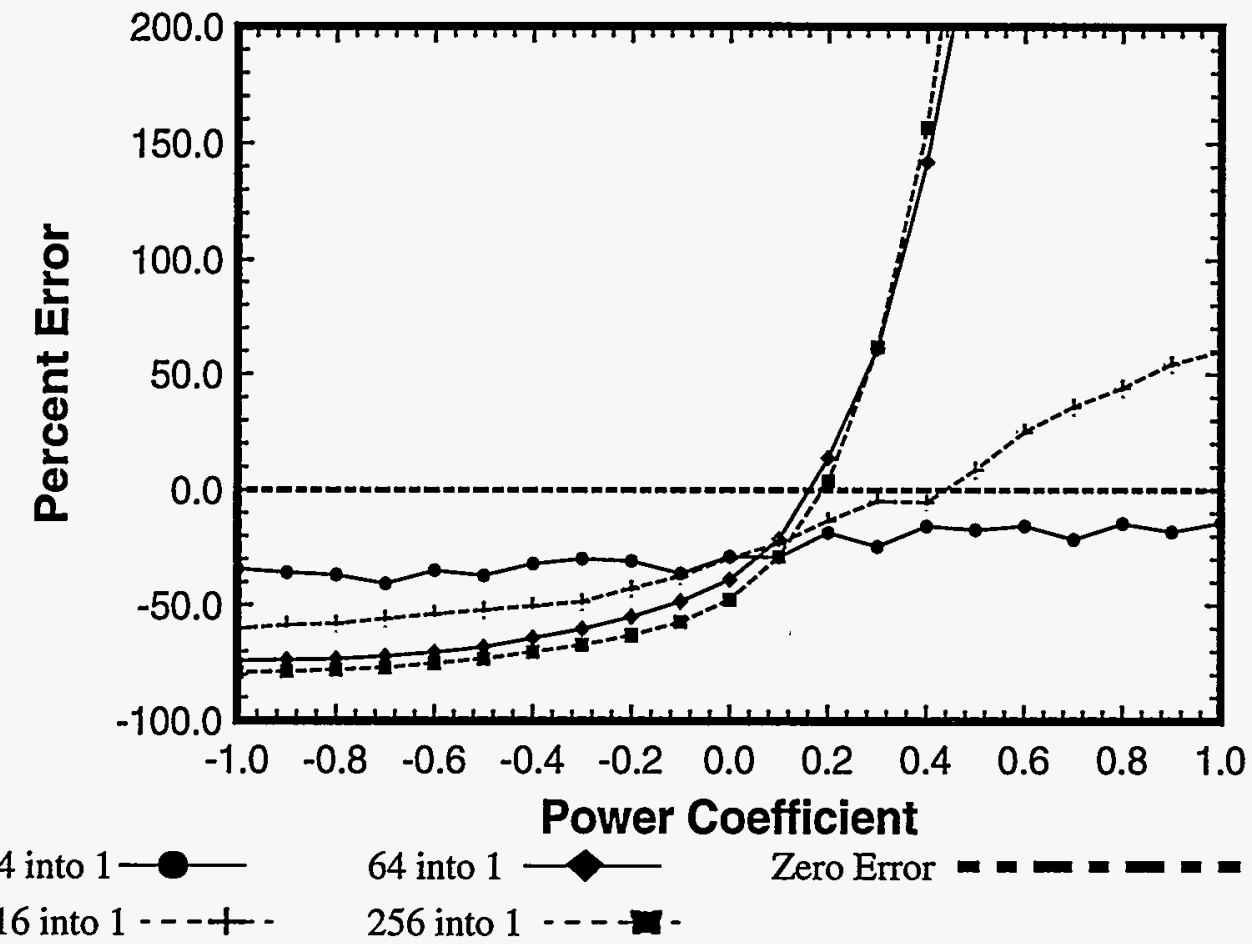

Figure 27. Graph showing percent error reproducing dispersivity through the simulated cross-sectional model of Yucca Mountain as a function of the power-law coefficient, $w$.

Reproduction of the point-scale longitudinal dispersion coefficient with progressive upscaling (figure 28) is somewhat similar to, but less extreme than that observed for dispersivity. As the dispersion coefficient is also a function of the mean particle velocity, the shapes of the power-coefficient/percent-error curves for dispersion coefficient are similar to those for mean par- 
ticle velocity (figure 25). Interestingly, the value of $w$ at which reproduction of the point-scale dispersion coefficient is closest appears to converge on zero, corresponding to the geometric average.

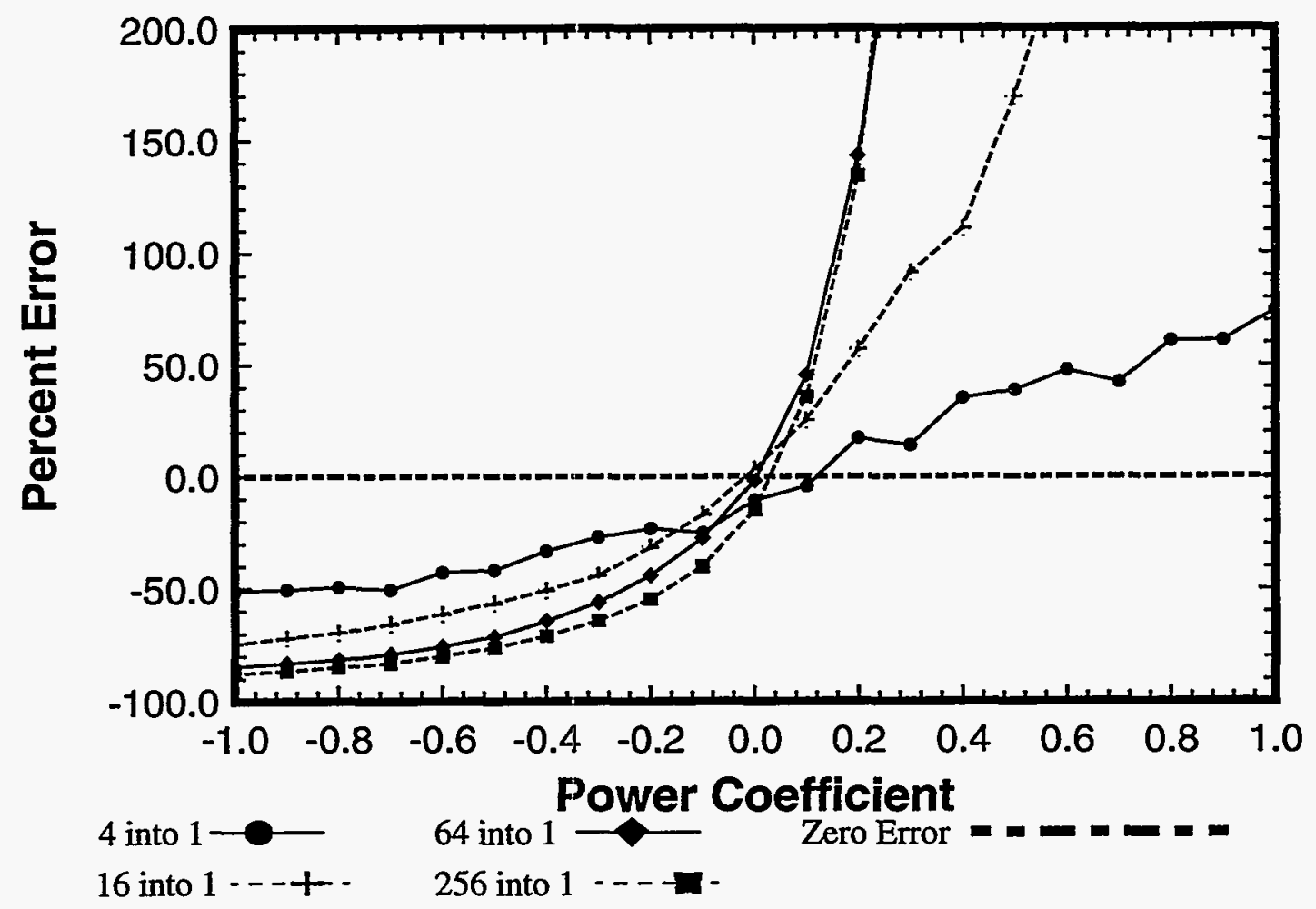

Figure 28. Graph showing percent error reproducing the longitudinal dispersion coefficient through the simulated cross-sectional model of Yucca Mountain as a function of the power-law coefficient, $w$.

In summary, a power coefficient below zero gives the best results for all parameters with the exception of dispersivity. This behavior is expected as the units are layered sub-orthogonal to the gradient. The large errors in reproducing the dispersivity and the dispersion coefficient with negative power coefficients indicate that the loss of information during upscaling is too great to accurately reproduce these parameters. For predictive use, dispersion must be modeled using a dispersivity parameter that takes into account the dispersion occurring at scales below that of the flow-model element scale. Based on the results of these numerical studies, a power coefficient of approximately -0.4 is suggested for use in the GWTT 95 calculations. 


\section{Discussion}

Numerical experiments using spatially correlated, isotropic fields of saturated hydraulic conductivity demonstrate that the majority of the proposed scaling methodologies predict similar decreases in the variance of the resulting populations of progressively upscaled, effective conductivity values. Notable exceptions to similarity of prediction are the spatial-averaging and renormalization techniques. These two algorithms overpredict the variability at smaller spatial correlation length-to-domain ratios, relative to other techniques. However, laboratory studies of collocated permeability measurements obtained at a variety of scales from slabbed samples of tuff (McKenna and Tidwell, 1995) have shown that variability of the measured larger-scale permeability values is generally greater than predicted using geometric averaging as the scaling algorithm. Thus, it may be possible to model the smaller physically measured decrease in variance using the spatial-averaging or renormalization techniques.

The spatial-averaging algorithm also performs poorly in reproducing the transport properties modeled in the numerical experiments involving the isotropic simulated conductivity fields. This poor performance is most likely caused by large overall variability of the simulations. For each transport measure, reproduction of the point-scale transport measures was relatively acceptable for smaller degrees of upscaling when the standard deviation of the point-scale conductivity values was equal to 0.5 . Increasing the standard deviation of the initial, point-scale field to 1.0 (most similar to Yucca Mountain field data) caused the spatial-averaging technique to produce completely unreasonably upscaled values in the numerical experiments.

The spatial-averaging algorithm, given by equation 8 , indicates that the scaled conductivity value is obtained by multiplying the geometric average $\left(K_{G}\right)$ of the point-scale values within the grid block by $e \cong 2.718$ raised to a power that involves a difference term. The difference term, $\left[\sigma^{2}-\gamma(V, V)\right]$, is computed from the variance $\left(\sigma^{2}\right)$ of the point-scale values within the grid block being upscaled and the average variogram value $[\gamma(V, V)]$ over that grid block. If the local variance of the point-scale values is large compared to the average variogram value, the exponen- 
tial term will become large. Because $K_{G}$ has been defined as $\log$ hydraulic conductivity and the values of $K_{G}$ are, in fact, negative numbers, the resulting $K_{e f f}$ is a large negative value, which corresponds to a very low estimate of effective hydraulic conductivity for the upscaled block.

This effect of the local variance is most noticeable when the ratio of element length to correlation length $\left(L_{e} / \lambda\right)$ is between 0.1 and 1.0. At ratios of $L_{e} / \lambda$ less than 0.1 , the variance of the contained point values will be small, as the point values being averaged are strongly correlated because the correlation length is at least ten times the size of the grid block. At $L_{e} / \lambda$ ratios above 1.0 , the upscaled grid block contains a sufficiently large number of point-scale values that the variance of those values tends towards the global variance.

This effect is illustrated in figure 29 , where the values calculated by the geostatistical method (equation 8) are compared to the values calculated by the Laplace solution. The handful of upscaled blocks with extremely small values of hydraulic conductivity cause the large overestimation of the dispersivity and the dispersion coefficient.

The renormalization technique for upscaling proposed by King (1989) also tends to create fields with a higher variance compared to other techniques [figures 15 through 17]. This higher variance is caused by the combination of arithmetic and harmonic averaging that takes place within the renormalization. These two types of averaging accentuate the high and low point values within the averaging space. Additionally, the renormalization calculation is limited to averaging $2 \times 2$-arrays of point values at a time. To upscale point values to larger blocks, several iterations of scaling $2 \times 2$ values are necessary to achieve the final level of scaling. This parsing of the total number of point values into $2 \times 2$ grids and scaling them separately causes the variance to be larger than the other methods where all points are averaged together at the same time.

The perturbation technique (equation 17) produces upscaled results that overestimate the flow parameters (figures 18 and 19) and produces transport parameters that are similar to the transport results of the other techniques (figures 20, 21 and 22). These results indicate that the per- 

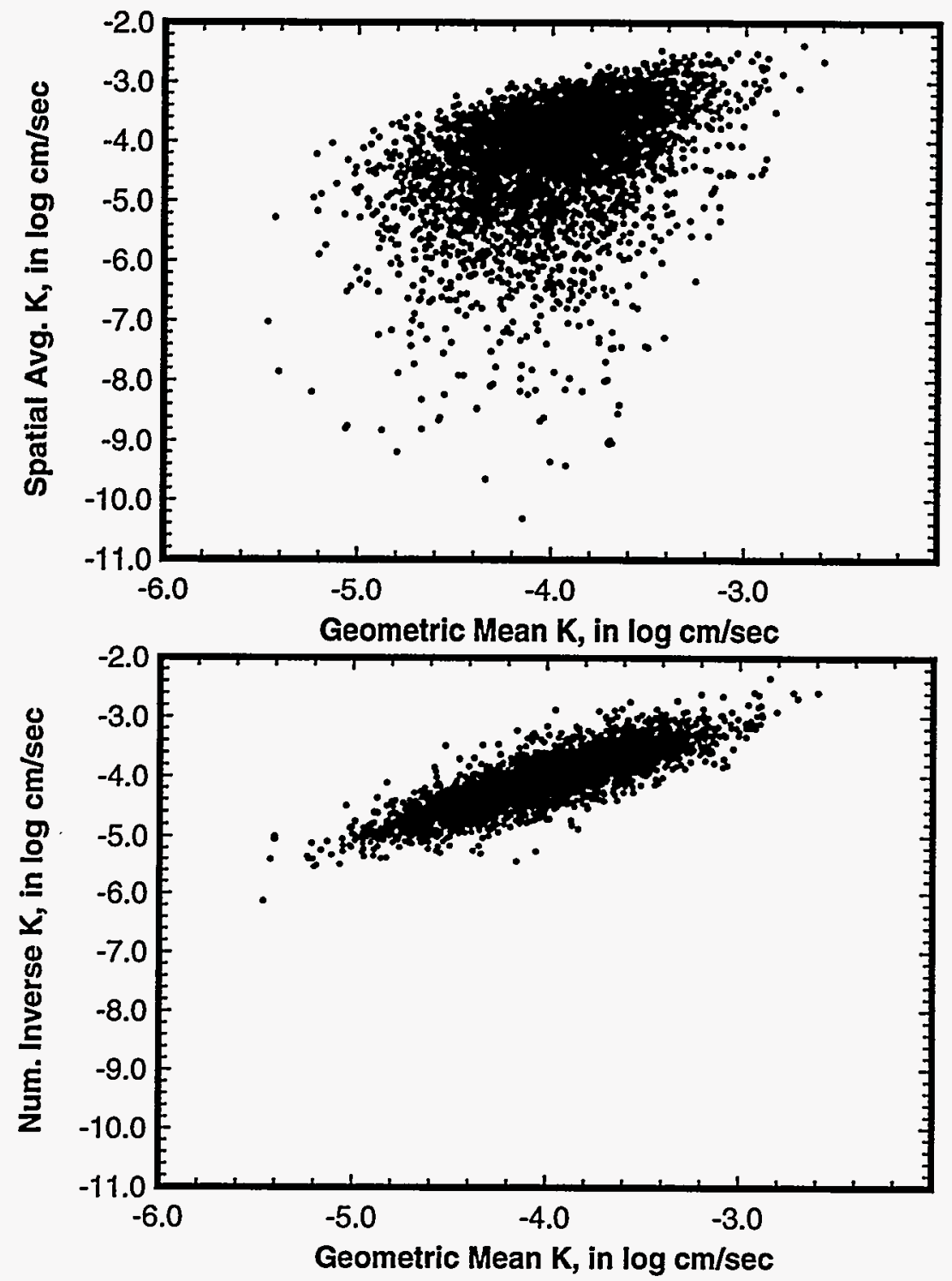

Figure 29. Comparison of hydraulic conductivity values upscaled by the spatial average and the geometric mean (top) for the field with a range of 20 and upscaled to elements with a length of $40 \times 40$. The bottom graph compares the geometric mean with the numerical inverse solution. Relative to other techniques, such as the numerical inverse solution, spatial averaging produces a larger variance and lower values.

turbation method is overestimating the mean value of hydraulic conductivity compared to other techniques, but it is calculating similar values for the variability of the upscaled hydraulic conductivity field.

It is possible to determine the value of dispersivity at various levels of upscaling necessary to retain the dispersion observed on the point scale fields. This dispersivity value is a function of 
the level of upscaling at element length to correlation length ratios below 1.0 and appears to approach an asymptotic value at element length to correlation length ratios greater than 1.0.

Selection of a scaling technique for application at Yucca Mountain to large-scale hydrologic models, such as that shown in figure 8, thus depends upon more-practical considerations of implementation. Specifically, the method used to produce the upscaled material-property values for the individual flow-model elements must be capable of handling the irregular shape of the adapted-grid polygons. This limitation effectively precludes practical implementation of the numerical-inverse method, which requires solving the Laplace equation in a highly irregular (and spatially varying) geometry. Geometric considerations also exclude practical use of renormalization; implementation of this scaling technique essentially mandates the use of square calculational elements (in two dimensions). Although the spatial-averaging scaling method could be applied to the irregular, polygonal grid of figure 9 , the computational burden of computing the average variogram value $(\gamma(V, V)$; eq. 8) for each individual grid cell effectively precludes use of this method as well.

Power-law averaging appears to be a fast, flexible and accurate technique for upscaling. The disadvantage of power-law averaging is the requirement of conducting a number of numerical experiments using different values of $w$ to determine the correct exponent. Results of powerlaw averaging in this study show that either travel-time distributions or total flux can be reproduced accurately by choosing the appropriate power. However, the most accurate power for reproducing the point-scale flux is not the most accurate power for reproducing the point scale transport parameters. In this situation, a compromise between reproducing the point-scale mass flux and the point-scale transport values must be reached. For example, in this study a power of approximately -0.6 reproduced the point scale mass flux but caused an error of roughly 10 to 15 percent in terms of reproducing the fastest particle velocities. Adjustment of the power to -0.4 would accurately reproduce the fastest particle velocities while causing only a 1 percent error in mass flux. 
Most of the proposed scaling methodologies (geometric mean, power-law averaging, renormalization and numerical inverse) can be used to scale hydraulic conductivity within a single geologic unit or in other applications for which a regular, upscaled flow-model grid is acceptable. However, the power-law averaging algorithm (including $w=-1,0$ and 1 ) is essentially the only scaling algorithm feasible for use in scaling irregular and possibly anisotropic flow-model grids, such have been proposed for use in the 1995 ground-water travel time calculations. Although use of power-law averaging requires numerical experimentation to determine the correct power value, the technique provides a fast, accurate, and flexible method of computing upscaled conductivity values.

The numerical experiments done in this study used a single-phase (saturated) flow code to examine the scaling behavior of saturated hydraulic conductivity. Many performance assessment calculations done within the Yucca Mountain project require the prediction of flow through the unsaturated portion of the potential repository block. Models used to make these predictions are dependent on knowledge of the saturated hydraulic conductivity in order to predict the behavior of hydraulic conductivity as a function of saturation. The correct scaling of the saturated hydraulic conductivity values is necessary for accurate determination of the relative hydraulic conductivity curve at the flow-model grid block scale.

It is also noted that the calculations of travel time done in this report are not directly comparable to travel times calculated through unsaturated porous media. The two major differences between a saturated medium and a partially saturated medium in terms of transport results will be the mean effective hydraulic conductivity and the effective heterogeneity of the medium. A partially saturated medium will have a lower effective hydraulic conductivity, and thus longer travel times, relative to the same medium at saturation. Additionally, the distribution of moisture within the unsaturated medium will create a different type and degree of heterogeneity relative to the saturated medium. Relatively small changes in saturation between adjacent flow model grid blocks can cause large changes in the relative hydraulic conductivity between these grid blocks, creating 
a larger degree of heterogeneity. Depending on the underlying porous medium, the heterogeneity caused by variable saturation may have a very short correlation length.

There are several specific Yucca Mountain Project applications for techniques examined in this study. First of all, estimation of the second moment (variance) of both saturated hydraulic conductivity and porosity distributions at a larger scale than the sample scale is necessary for the 1-D travel time calculations done in previous TSPA efforts (Wilson and others, 1994). The example volume-variance results presented in table 1 of this report show that the variance of a distribution for a property that scales arithmetically (e.g., porosity) can be predicted accurately from the point-scale statistics. Secondly, the numerical experiments show how the variance of point-scale measurements of saturated hydraulic conductivity scale. Predictions of the upscaled variability for both saturated hydraulic conductivity and porosity will be used in the creation of geostatistical simulations of these properties for the GWTT 95 effort. Power-law averaging will also be used in the GWTT 95 effort to determine upscaled values of saturated hydraulic conductivity at the flow model grid-block scale.

\section{Conclusions}

The numerical experiments described in this report provide several conclusions that are relevant to scaling of properties at Yucca Mountain. The first and second moments of an upscaled distribution of an additive property, such as porosity, can be estimated from the point-scale distribution and knowledge of the point scale variogram through volume variance relationships developed in the mining industry. Scaling techniques for hydraulic conductivity based on theory requiring small varaiances and exponential variograms (e.g., spatial averaging) do not apply to the data observed at Yucca Mountain. Power-law averaging provides a fast and flexible means by which to upscale properties from the point scale to the flow-model grid block scale. However, power-law averaging is dependent on determination of the correct power coefficient through numerical experiments. Although power-law averages are empirical techniques, they are not based on the equations of continuity or motion of fluid flow, they are shown to be very accurate. 


\section{References}

Barnard, R.W. and H.A. Dockery, (eds.), 1991, Technical summary of the Performance Assessment Calculational Exercises for 1990 (PACE-90), Volume 1: "Nominal configuration" hydrogeologic parameters and calculational results: Sandia Report SAND90-2726, Sandia National Laboratories, Albuquerque, N. Mex., 189 p.

Barnard, R.W., M.L. Wilson, H.A. Dockery, J.H. Gauthier, P.G. Kaplan, R.R. Eaton, F.W. Bingham, and T.H. Robey, 1992, TSPA 1991: An initial total-system performance assessment for Yucca Mountain: Sandia Report SAND91-2795, Sandia National Laboratories, Albuquerque, N. Mex., 376 p.

Clark, I. 1979, Practical Geostatistics, Elsevier Applied Science Publishers, New York, 129 pp.

Dagan, G., 1979, Models of groundwater flow in statistically homogeneous porous formations: Water Resources Research, v. 15, p. 47-63.

Dagan, G., 1989, Flow And Transport in Porous Formations, New York: Springer-Verlag, 466 p.

Desbarats, A.J., 1987, Numerical estimation of effective permeability in sand-shale formations: Water Resources Research, v. 23, p. 273-286.

Desbarats, A.J., 1992, Spatial averaging of hydraulic conductivity in three-dimensional heterogeneous porous media, Mathematical Geology, v. 24, no. 3, pp. 249-267.

Desbarats, A.J., 1994, Geostatistical analysis of water saturation in heterogeneous unsaturated media under capillary-gravity equilibrium, in: Geostatistics for the Next Century, R. Dimitrakopoulos (ed.), Boston: Kluwer Academic Publishers, p. 359-370.

Desbarats, A.J. and R. Dimitrakopoulos, 1990, Geostatistical modeling of transmissibility for 2D reservoir studies: SPE Formation Evaluation, v. 5, p. 437-443.

Deutsch, C.V. 1989a, Calculating effective absolute permeability in sandstone/shale sequences: SPE Formation Evaluation, v. 4, p. 343-348.

Deutsch, C.V., 1989b, DECLUS: A Fortran 77 program for determining optimum spatial declustering weights: Computers and Geosciences, v. 15, p. 325-332.

Deutsch, C.V. and A.G. Journel, 1992, GSLIB: Geostatistical Software Library and User's Guide, New York: Oxford University Press, 340 p. (NNA.930507.0081)

Dimitrakopoulos, R. and A.J. Desbarats, 1993, Geostatistical modeling of grid block permeabilities for 3D reservoir simulators: SPE Reservoir Engineering, v. 8, p. 13-18.

Dudley, A.L., R.R. Peters, J.H Gauthier, M.L. Wilson, M.S. Tierney, and E.A. Klavetter, 1988, Total system performance assessment code (TOSPAC) Volume 1: Physical and mathematical bases: Sandia Report SAND85-0002, Sandia National Laboratories, Albuquerque, N. Mex., 204 p.

Duquerroix, J-P. L., P. Lemouzy, B. Noetinger, and R.K. Romeu, 1994, Influence of the permeability anisotropy ratio on large-scale properties of heterogeneous reservoirs, Reservoir Engineering Proceedings, SPE Annual Technical Conference and Exhibition, Houston, SPE Paper 26612, Richardson, TX: Society of Petroleum Engineers, 16 p.

Dykaar, B.B. and P.K. Kitanidis, 1992a, Determination of the effective hydraulic conductivity for heterogeneous porous media using a numerical spectral approach. 1. Method: Water Resources Research, 
v. 28, p. $1155-1166$.

Dykaar, B.B. and P.K. Kitanidis, 1992b, Determination of the effective hydraulic conductivity for heterogeneous porous media using a numerical spectral approach. 2. Results: Water Resources Research, v. 28, p. 1167-1178.

Dykaar, B.B. and P. K. Kitanidis, 1993, Transmissivity of a heterogeneous formation: Water Resources Research, v. 29, p. 985-1001.

Ewing, R.P. and D.B. Jaynes, 1995, Issues in single-fracture transport modeling: scales, algorithms and grid types: Water Resources Research, v. 31, p. 303-312.

Ferrand, L.A. and M.A. Celia, 1992, The effect of heterogeneity on the drainage capillary pressure-saturation relation: Water Resources Research, v. 28, p. 859-870.

Flint, A.L., L.E. Flint and K.A. Richards, 1994, Evaluation of measurement scale using imbibition experiments in volcanic tuffs: Soil Science Society of America Journal, v. 58, p. 94-102.

Flint, L.E., A.L. Flint, and C.A. Rautman, 1996, Physical and hydrologic properties of rock outcrop samples at Yucca Mountain, Nevada, USGS/OFR-95-280, U.S. Geological Survey, Denver, Colorado.

Freeze, R.A., 1975, A stochastic-conceptual analysis of one-dimensional groundwater flow in a nonuniform homogeneous media: Water Resources Research, v. 11, p. 725-741.

Freeze, R.A. and J.A. Cherry, 1979, Groundwater, Prentice Hall Publishing Co., Englewood Cliffs, New Jersey, $604 \mathrm{pp}$.

Freeze, R.A., J. Massmann, L. Smith, T. Sperling, and B. James, 1990, Hydrogeological decision analysis: 1. A framework, Ground Water, v. 28, pp. 738-766.

Gelhar, L.W., 1986, Stochastic subsurface hydrology: From theory to applications, Water Resources Research, v. 22, pp. 135S-145S.

Gelhar, L.W.,1993, Stochastic Subsurface Hydrology, Englewood Cliffs, New Jersey: Prentice Hall, 390 p.

Gelhar, L.W. and C.L. Axness, 1983, Three-dimensional stochastic analysis of macrodispersion in aquifers, Water Resources Research, v. 19, p. 161-180.

Gomez-Hernandez, J.J. and A.G. Journel, 1994, Stochastic characterization of grid-block permeabilities, in: Stanford Center for Reservoir Forecasting Report 7, Stanford University, Stanford, California, $21 \mathrm{p}$.

Goode, D.J., and A.M. Shapiro, 1991, Comment on "Flow and tracer transport in a single fracture: A stochastic model and its relation to some field observations" by L. Moreno et al., Water Resources Research, v. 14, pp. 129-132.

Gutjahr, A.L., L.W. Gelhar, A.A. Bakr, and J.R. MacMillan, 1978, Stochastic analysis of spatial variability in subsurface flows. 2. Evaluation and application: Water Resources Research, v. 14, p. 953-959.

Hill, M.C., 1992, A computer program (MODFLOWP) for estimating parameters of a transient, threedimensional, groundwater flow model using nonlinear regression, U.S. Geological Survey OpenFile Report 91-484, 358 p.

Indelman, P., 1993, Upscaling of permeability of anisotropic heterogeneous formations. 3. Applications: Water Resources Research, v. 29, p. 935-943. 
Isaaks, E.H. and R.M. Srivastava, 1989, Applied Geostatistics, New York: Oxford University Press, 561 p.

Istok, J.D., C.A. Rautman, L.E. Flint, and A.L. Flint, 1994, Spatial variability in hydrologic properties of a volcanic tuff, Ground Water, v. 32, p. 751-760.

Jensen, J.L., 1991, Use of the geometric average for effective permeability estimation, Mathematical Geol$o g y$, v. 23, p. 833-840.

Journel, A.G., 1983, Nonparametric estimation of spatial distributions: Mathematical Geology, v. 15, p. $445-468$

Journel, A.G., 1994, Conditional simulation of geologically-averaged block transmissivities, in: Stanford Center for Reservoir Forecasting Report 7, Stanford University, Stanford, California, 19 p.

Journel, A.G., C.V. Deutsch, and A.J. Desbarats, 1986, Power averaging for block effective permeability, 54th California Regional Meeting, Oakland, CA, SPE Paper 15128, Richardson, TX: Society of Petroleum Engineers, p. 329-334.

Journel, A.G. and Ch. J. Huijbregts, 1978, Mining Geostatistics, New York: Academic Press, 600 p.

Kaplan, P.G., 1991, A formalism to generate probability distributions for performance assessment modeling, in: High Level Radioactive Waste Management, Proceedings of the Second Annual International Conference, April 28-May 3, American Nuclear Society, La Grange Park, Ill., p. 1487-1490.

Kaplan, P.G., 1993, Pre-waste-emplacement ground-water travel time sensitivity and uncertainty analyses for Yucca Mountain, Nevada: Sandia Report SAND92-0461, Sandia National Laboratories, Albuquerque, N. Mex., 121 p.

Kaplan, P.G., and L. Yarrington, 1989, Modeling the uncertainties in the parameter values of a layered, variably saturated column of volcanic tuff using the beta probability distribution, in: Solving Ground Water Problems with Models, Fourth International Conference, February 7-9, National Water Well Association, Dublin, OH, p. 111-128.

Keuper, B.H. and D.B. McWhorter, 1992, The use of macroscopic percolation theory to construct largescale capillary pressure curves, Water Resources Research, v. 28, p. 2425-2436.

King, P.R., 1989, The use of renormalization for calculating effective permeability, Transport in Porous Media, v. 4, p. 37-58.

Kitanidis, P.K., 1990, Effective hydraulic conductivity for gradually varying flow, Water Resources Research, v. 26, p. 1197-1208.

Le Loc'h, G., 1989, An efficient strategy for combining the permeabilities: Practical application on a simulated reservoir, in: Geostatistics; Proceedings of the Third International Geostatistics Conference, Avignon, France, Dordrecht, The Netherlands, Kluwer Academic Press. p. 557-568.

Lin, Y.T., M.S. Tierney, and S. Sinnock, ed., 1986, Preliminary estimates of groundwater travel time and radionuclide transport at the Yucca Mountain repository site: Sandia Report SAND85-2701, Sandia National Laboratories, Albuquerque, N. Mex., 84 p.

McDonald, M.G. and A:W. Harbaugh, 1988, A modular three-dimensional finite-difference ground-water flow model, U.S. Geological Survey Techniques of Water-Resources Investigations, Book 6, TWI 06-A1, Chapter A1. Denver, CO: U.S. Geological Survey. 
McKenna, S.A. and C.A. Rautman, 1995, Summary evaluation of Yucca Mountain surface transects with implications for downhole sampling, Sand Report SAND 94-2038, Sandia National Laboratories, Albuquerque, N. Mex., 45 p.

McKenna, S.A. and V.C. Tidwell, 1995, Numerical and physical testing of upscaling techniques for constitutive properties, in: High Level Radioactive Waste Management, Proceedings of the Sixth AnnualInternational Conference, April 30-May 5, American Nuclear Society, La Grange Park, Ill., p. 6768.

Moreno, L. and C-F. Tsang, 1994, Flow channeling in strongly heterogeneous porous media: a numerical study: Water Resources Research, v. 30, p. 1421-1430.

Moreno, L., Y.W. Tsang, C.F. Tsang, F.V. Hale, and I. Neretnieks, 1988, Flow and tracer transport in a single fracture: A stochastic model and its relation to some field observations, Water Resources Research, v. 24, pp. 2033-2048.

Piggott, A.R. and D. Elsworth, 1992, Analytical models for flow through obstructed domains: Journal of Geophysical Research, v. 97 (B2), p. 2085-2093.

Pollock, D.W., 1988, Semianalytical computation of path lines for finite-difference models, Ground Water, v. $26(6)$, pp. $743-750$.

Pollock, D.W., 1989, Documentation of computer programs to compute and display pathlines using results from the U.S. Geological Survey modular three-dimensional finite-difference ground-water flow model: U.S. Geological Survey Open-File Report 89-381, 188 p.

Rautman, C.A. and A.L. Flint, 1992, Deterministic geologic processes and stochastic modeling, in: High Level Radioactive Waste Management, Proceedings of the Third Annual International Conference, April 12-16, 1992, American Nuclear Society, La Grange Park, Ill., p. 1617-1624.

Rautman, C.A. and T.H. Robey, 1993, Recent developments in stochastic modeling and upscaling of hydrologic properties in tuff, in: High Level Radioactive Waste Management, Proceedings of the Fourth Annual International Conference, April 26-30, 1993, American Nuclear Society, La Grange Park, Ill., p. 1437-1445.

Robey, T.H., 1993, Numerical methods for fluid flow in unsaturated heterogeneous tuff, in: High-Level Radioactive Waste Management, Proceedings of the Fourth International Conference, April 26-30, 1993, American Nuclear Society, La Grange Park, Ill., p. 138-145.

Robey, T.H., 1994a, Development of models for fast fluid pathways through unsaturated heterogeneous porous media, Contractor Report SAND93-7109, Sandia National Laboratories, Albuquerque, N. Mex., 104 p.

Robey, T. H., 1994b, Modeling heterogeneous unsaturated porous media flow at Yucca Mountain, in: High-Level Radioactive Waste Management, Proceedings of the Fifth Annual International Conference, May 22-26, 1993, American Nuclear Society, La Grange Park, Ill., p. 2678-2684.

Rubin, Y. and J.J. Gomez-Hernandez, 1990, A stochastic approach to the problem of upscaling of hydraulic conductivity in disordered media: Theory and unconditional numerical simulations, Water Resources Research, v. 26, p. 691-701.

Rutherford, B.M., I.J. Hall, R.R Peters, R.G. Easterling, and E.A. Klavetter, 1992, Statistical analysis of hydrologic data for Yucca Mountain, Sandia Report SAND87-2380, Sandia National Laboratories, Albuquerque, N. Mex., 273 p. 
Scott, R.B. and J. Bonk, 1984, Preliminary geologic map of Yucca Mountain, Nye County, Nevada, with geologic sections, U.S. Geological Survey Open-File Report 84-494.

Sisson, J.B. and P.J. Wierenga, 1981, Spatial variability of steady-state infiltration rates as stochastic processes, Soil Science Society of America Journal, v. 45, p. 699-704.

Tidwell, V.C., 1994, Laboratory investigation of constitutive property scaling behavior, Formation Evaluation and Reservoir Geology, Proceedings of the I994 SPE Annual Technical Conference and Exhibition, Sept. 25-28th, New Orleans, SPE 28456, Society of Petroleum Engineers, Richardson TX, p. 947-957.

Tidwell, V.C., (in press), Laboratory investigation of constitutive property upscaling in volcanic tuffs, Sandia Report SAND 95-1888, Sandia National Laboratories, Albuquerque, N. Mex.

Tran, T., 1994, Direct simulation of block effective properties: inference and conditioning, in: Stanford Center for Reservoir Forecasting, Report 7, Stanford University, Stanford, California, 26 p.

Walpole, R.E. and R.H. Myers, 1989, Probability and Statistics for Engineers and Scientists, 4th ed., New York: MacMillan Publishing Co., 765 p.

Warren, J.E. and H.S. Price, 1961, Flow in Heterogeneous Media: SPE Journal, v. 1, p. 153-169.

Wheatcraft, S.W. and S.W. Tyler, 1988, An explanation of scale-dependent dispersivity in heterogeneous aquifers using concepts of fractal geometry: Water Resources Research, v. 24, p. 566-578.

Wilson, M.L., J.H. Gauthier, R.W. Barnard, G.E. Barr, H.A. Dockery, E. Dunn, R.R. Eaton, D.C. Guerin, N. Lu, M.J. Martinez, R. Nilson, C.A. Rautman, T.H. Robey, B. Ross, E.E. Ryder, A.R. Schenker, S.A. Shannon, L.H. Skinner, W.G. Halsey, J.D. Gansemer, L.C. Lewis, A.D. Lamont, I.R. Triay, A. Meijer, and D.E. Morris, 1994, Total-system performance assessment for Yucca Mountain - SNL second iteration (TSPA-1993), Volumes 1 and 2, Sandia Report SAND93-2675, Sandia National Laboratories, Albuquerque, N. Mex., 376 p.

Zimmerman, R.W., G.S. Bodvarsson, A.L. Flint, and L.E. Flint, 1993, An inverse procedure for estimating the unsaturated hydraulic conductivities of volcanic tuffs, in: High Level Radioactive Waste Management, Proceedings of the Fourth Annual International Conference, April 26-30, American Nuclear Society, La Grange Park, Ill., p. 1052-1058. 


\title{
APPENDIX
}

\author{
Literature Review for \\ Averaging Properties of Arbitrary Rock Volumes
}

Sandia Contractor Report

Submitted by:

Kadri Dagdelen

Leslie Gertsch

Mining Engineering Department

Colorado School of Mines 


\section{INTRODUCTION}

This report surveys the recent literature regarding methods of determining the most representative average value of a physical property, or parameter, for an arbitrary volume of rock. The purpose is to calculate such average values for incorporation into a numerical model of the rock mass at the Yucca Mountain site.

\section{Background}

Yucca Mountain is the location of a proposed underground repository for highlevel nuclear waste in volcanic tuffs of the Basin-and-Range physiographic province of the United States. It is situated approximately two hours north of Las Vegas, Nevada.

The U.S. Department of Energy (DOE) is charged with characterization of the Yucca Mountain site to determine its suitability for this purpose. Part of that study is the creation of a numerical model of the rock mass that would enclose the repository. Such a numerical model contains sub-volumes, or elements, within each of which the physical properties of the rock are assumed to be uniform. The model will be used to predict the response of the rock mass to conditions imposed by the presence of the repository and its contained waste over the expected life of the repository.

The physical properties of these elements must be calculated from a sparse network of measurements performed throughout the area surrounding Yucca Mountain. Each type of measurement is the result of different techniques and reflects the aggregate response of different volumes of rock and other materials (water, air, mineral constituents) to the measurement process. Accordingly, the averaging of values obtained from different locations, and by different methods, is not necessarily straightforward.

\section{Scalar vs. Vector Quantities}

Many of the rock properties to be modeled are scalars, i.e. they are described fully by simple numerical magnitudes (e.g. thermal conductivity, density, etc.). However, some must be considered vectors. These require a direction in addition to a magnitude (e.g. principal conductivity or stress vectors) for complete description. Many scalar parameters vary in magnitude with the direction of measurement (e.g. strength, modulus, etc.). This rock mass property is known as anisotropy. Obviously, the last two situations require more information to average accurately over a volume, and therefore are more difficult to deal with mathematically. 


\section{DISCUSSION}

A great many papers have been published in the last twenty years that touch on the subject of this investigation. Some are more pertinent to the present subject than others; most have something to contribute, even if not their main conclusions. To assist in this effort, the papers have been grouped into categories. A paper may be included in more than one category. Bibliographic information and abstracts of pertinent papers are listed in the Appendices.

The most directly useful papers are discussed briefly here. Several approaches will be available to average any given parameter. The next step will be to select the most appropriate and begin coding.

Note that this report does not mark the end of the literature review process. Review will continue throughout the research, to keep abreast of the evolution of the various fields that affect this study.

\section{Geostatistics}

Since its development over twenty years ago, geostatistics has been used in areas beyond ore reserve estimation. This section includes recent work in the application of geostatistics to problems in geomechanics, particularly physical property estimation.

Three reference books are included here as fundamental to the understanding and application of geostatistics to any field (David, 1977; Journel and Huijbregts, 1978; and Matheron, 1971). Scalar rock mechanics properties have been subjected to increasingly sophisticated geostatistical analysis (La Pointe, 1980; Pineau and Thomas, 1983; Hoerger and Young, 1987)); more recently, indicator kriging has been applied to three-dimensional vector information, such as joint orientation (Young, 1987b; Young and Hoerger, 1988). Scalar joint information also is being analyzed geostatistically (Villaescusa and Brown, 1990).

\section{Modeling of Jointed Rock}

These papers deal with approaches to modeling or characterizing rock that contains joints, fractures, and other discontinuities. This area of the literature is large, and is only briefly sampled here. The subject is included due to the effect joints have on the in situ values of rock physical properties, rather than to assist creation of the numerical model itself.

Most of the papers in this group are concerned with various modeling approaches to joint networks (e.g. Kulatilake, 1988; Lin and Fairhurst, 1991; Cai and Horii, 1993) and scale effects on modeling of joint mechanical behavior (e.g. Muralha and Pinto da Cunha, 1990a; Sarra Pistone, 1990). Some authors have attempted to relate rock mass quality to different indicators of jointing, such as Kazi 
and Sen, 1985 and Sen, 1990a, 1990b. These measures then could be used to reduce the magnitudes of physical properties accordingly (see Elastic Properties section). The expanding field of fractal geometry is being applied to the description of jointed rock (e.g. Sen, 1992), as is the more traditional approach of topology (e.g. Lin and Fairhurst, 1991).

\section{Indirect or Inverse Approaches}

This section includes analysis techniques developed for properties other than those of interest to this project. However, they could conceivably be adapted for this use, if no more direct methods are found.

Moris and Oravecz, 1985 discuss a procedure for calculating the properties of regions that are smaller than the volumes of influence of physical property measurements. This method would require the running of several coarse models before the final model could be created. Rajaram and McLaughlin, 1990 treat variability in hydrologic properties as the result of the superposition of random residuals at different scales and estimate spatial trends accordingly.

Inverse approaches offer an alternative to direct, forward estimation of physical properties, but they also add additional steps to the process. Such approaches also require certain information before they can be used. Hoeksema and Kitanidis, 1984 and Long et al., 1991 illustrated this for hydrological models.

\section{Hydrological Properties}

These papers are concerned with estimating hydrological parameters, such as permeability, transmissivity, porosity, etc. in jointed rock. Again, this large area of the literature is not sampled in depth here. Rather, a representative selection has been chosen from which further works can be accessed if necessary.

Phillips and Wilson, 1989 developed a technique to estimate correlation scale of hydraulic conductivity to reduce reliance on many data measurements. Neuman, 1990 discussed a somewhat different approach to scaling of transport phenomena that was not developed mathematically enough in the paper to implement directly.

\section{Elastic Properties, Thermal Conductivity, and Strength Properties}

The papers in this group discuss approaches to estimating rock mass physical properties using various types of input data. Elastic properties include Young's modulus and Poisson's ratio. Thermal conductivity is an important parameter in performance assessment of a rock mass containing heat-producing waste. Strength properties include uniaxial compressive strength and tensile strength (both direct and indirect). Density is included in this category as well. 
Most of the published reports in this area are focused on constitutive models, whether conceptual, numerical, or analytical (e.g., Pariseau, 1993; Bauer et al., 1985). Others relate various joint parameters to rock mass deformability

(Grossmann, 1990) or elastic properties (Hu and Huang, 1993; Pariseau and Moon, 1988).

Thermal conductivity (Van Stone, 1985) has been calculated successfully from the properties of structural aggregates, a procedure that could be applied to rock masses if enough is known about the spatial thermal property variation.

Much work has been done on the effects of scale and data sparseness on rock stress measurements. Some of those results are sampled here (e.g. Haimson, 1990; Martin et al., 1990; Cuisiat and Haimson, 1992). Fadeev, 1990 and Gunsallus and Kulhawy, 1984 investigated aspects of standard measures of rock strength, including scale effects and the variations associated with each type of measurement. Miller and Luark, 1993 applied a Markov-Bayes technique to the spatial simulation of rock strength.

\section{Volumetric Averaging Techniques}

These approaches are almost directly applicable to the present subject. In this case, the arbitrary volumes are the elements of the finite element model. Some studies follow the representative elemental volume (REV) approach. However, the REV for a given rock mass may be larger than a given model element, smaller, or it may not exist at all. Nevertheless, many attempts have been made to determine the size of the REV from various information sources (e.g. Cai and Horii, 1993; Heuze, 1980; Grossmann, 1980).

Lundquist et al., 1989 also used a Bayesian approach to include "soft" data and non-local information into site characterization studies. Pine et al., 1990 provide the results of a field study comparing stress measurements made using techniques with different volumes of influence. Peres-Rodrigues, 1983 developed a statistical method to deal with the effects of multiple parameters acting simultaneously within a rock mass.

\section{General Relationships}

This group of papers proposes to elucidate generic relationships of rock physical properties to spatial location. Such relationships would assist in determining the most representative single value of a parameter given certain known quantities about the model element in question.

In addition to constitutive laws, this section also includes less commonly used methods, such as fuzzy sets (Nguyen and Ashworth, 1985), fractals (Shurtz, 1992), and the method of extremes (Yegulalp and Kim, 1992). 


\section{Case Studies}

The least directly applicable to this study, case studies nevertheless illustrate how well or how poorly certain approaches reflect the true aggregate properties of a rock mass. Their importance lies in direct physical validation, the importance of which cannot be overemphasized.

Case studies have been performed all over the world, and include work at Stripa (Long et al., 1991), Wales (Pine et al., 1990), the LNEC (Pinto da Cunha and Muralha, 1990), and the Yucca Mountain site (Zimmerman and Blanford, 1985).

\section{Miscellaneous}

The miscellaneous group contains those papers that appear useful, but do not fit well within any of the other divisions. This includes papers regarding physical property measurements at the Yucca Mountain site (Price and Bauer, 1985).

\section{SUMMARY}

Further searching would undoubtedly uncover more research with application to this study; however, the present database is sufficient to begin the programming process. Specific references will be retrieved as they are needed. 


\section{Appendix.1: Geostatistics}

Cyrul, T. and K.F. Unrug, 1985, Geostatistical approach to roof fall prediction, Research \& Engineering Applications in Rock Masses, Proceedings of the 26th U.S. Symposium on Rock Mechanics, June 26-28, 1985, Rapid City, SD, E. Ashworth (ed.), Boston, MA: A.A. Balkema, p. 967-975.

This paper presents a new approach to characterization of mine roof behavior. No assumptions concerning rocks or roof conditions are made, except to assume that the measuring equipment performs properly. The approach is based on mine roof monitoring over a large area, and is derived from basic geostatistics.

David, M.,1977, Geostatistical Ore Reserve Estimation, New York, Elsevier Scientific Pub. Co. $364 \mathrm{p}$.

reference book

Hoerger, S.F. and D.S. Young, 1987, Predicting local rock mass behavior using geostatistics, Rock Mechanics: Proceedings of the 28th U.S. Symposium, June 29-July 1, 1987, Tucson, AZ, I.W. Farmer, J.J.K. Daemen, C.S. Desai, C.E. Glass, and S.P. Neuman (eds.), Boston, MA: A.A. Balkema, p. 99-106.

Geostatistics can be used to make local estimates of spatially correlated rock mass properties. Local estimates, used as design inputs, allow a geotechnical design to make local adjustments to locally varying rock mass conditions. The use of geostatistics for local estimation of design inputs is illustrated using rock joint orientation data for slope stability analysis of a surface mine. The local inputs suggest local designs which are significantly different from the design made using site-wide averages composited from all sampled locations within the mine.

Journel, A.G., Ch.J. Huijbregts, 1978, Mining Geostatistics, New York: Academic Press, 600 pp.

reference book

La Pointe, P.R., 1980, Analysis of the spatial variation in rock mass properties through geostatistics, Rock Mechanics: A State of the Art, Proceedings, 21 st Symposium on Rock Mechanics, May 28-30, 1980, University of Missouri-Rolla, D.A. Summers (comp.), Rolla, MO: University of Missouri, p. 570-580.

Accurate knowledge of the spatial distribution of rock mass properties enhances the design of safe and economic rock structures. In this paper, geostatistics is adapted 
to predict the scalar, vectorial, and tensorial rock properties important to rock engineering. Geostatistical analysis makes it possible to:

(1) Formulate a relatively simple yet accurate mathematical model of a statistically inhomogeneous rock mass from limited sample data.

(2) Determine how far into a rock mass sample data can be validly extrapolated.

(3) Design sampling patterns that maximize information while minimizing cost.

(4) Predict mechanical properties in a variable rock mass from sample values with a minimum error of estimation.

A geostatistical analysis of jointing in a quarry confirms the potential of this method for characterizing the properties of a variable and inhomogeneous rock mass. Geostatistics indicates the degree of inhomogeneity in the frequencies and orientations of two distinct joint sets, and estimates the distance to which these properties can be extrapolated. Additionally, the results suggest that each joint set can be represented by a regional semivariance function plus a more local oscillatory component corresponding to the avg. spacing of the most persistent joints. Such a model is useful for design of economic sampling schemes and for realistic numerical modeling of rock mass mechanics.

La Pointe, P.R., 1981, Improved numerical modeling of rock masses through geostatistical characterization, Rock Mechanics from Research to Application, 22nd U.S. Symposium on Rock Mechanics, June 29-July 2, 1981, Cambridge, MA, H.H. Einstein (comp.), Cambridge, MA: Massachusetts Institute of Technology, p. 416421.

NA. Estimation of modulus, Poisson's ratio, and cohesion, plus jointing traits, were supplemented by geostatistical analysis of core samples and surface studies to give semivariograms of these parameters.

Matheron, G., 1971, The theory of regionalized variables and its applications, Les Cahiers du Centre de Morphologie Mathematique de Fontainebleau No. 5, Paris: Ecole nationale superieure des mines, $211 \mathrm{p}$.

Neuman, Shlomo P., 1990, Universal scaling of hydraulic conductivities and dispersivities in geologic media, Water Resources Research, Vol. 26, No. 8, pp. 1749-1758.

An interpretation is offered for the observation that dispersivities increase w/ scale. Apparent longitudinal dispersivity data from a variety of hydrogeologic settings are assumed to represent a continuous hierarchy of log hydraulic conductivity fields w/ mutually uncorrelated increments, each field having its own exponential autocovariance, associated integral scale, and variance that increases as a power of scale. Such a hierarchy is shown theoretically to form a self-similar random field w/ homogeneous increments. Regardless of whether or not the underlying assumption is valid, one can justify interpreting the apparent dispersivities in a manner consistent w/ a recent quasi-linear theory of non-Fickian and Fickian 
dispersion in homogeneous media which supports the notion of a self-similar hierarchy a posteriori. The hierarchy is revealed to possess a semivariogram gamma(s) $=\operatorname{cs}^{\wedge} 0.5$, where $c$ is a constant, and a fractal dimension $\mathrm{D}=\mathrm{E}-0.75$, where $\mathrm{E}$ is the topological dimension of interest. This can be viewed as a universal scaling rule about which large deviations occur due to local influences including the existence of discrete natural scales at which log hydraulic conductivity is statistically homogeneous. As such homogeneity is at best a local phenomenon occurring intermittently over narrow bands of the scale spectrum, one must question the utility of associating medium properties with representative elementary volumes and relying on Fickian models of dispersion over more than relatively narrow scale intervals. Porous and fractured media appear to follow the same idealized scaling rule for both flow and transport, raising a question about the validity of many distinctions commonly drawn between such media. Finally, the data suggest that conditioning transport models through calibration against hydraulic measurements has the effect of filtering out large-scale modes from the hierarchy.

Phillips, F. M., J. L. Wilson, 1989, An approach to estimating hydraulic conductivity spatial correlation scales using geological characteristics, Water Resources Research, Vol. 25, No. 1, pp. 141-143.

Pineau, A. and A. Thomas, 1983, Regionalisation des proprietes mecaniques des roches; Approche structurale geostatistique $=$ Regionalization of mechanical properties of rocks; geostatistical structural approach, Fifth International Congress on Rock Mechanics, Melbourne, Australia, Boston, MA: A.A. Balkema, p. B23-B27.

Villaescusa, E. and E.T. Brown, 1990, Characterizing joint spatial correlation using geostatistical methods, International Symposium on Rock Joints, June 4-6, 1990, Loen, Norway, N. Barton and O. Stephansson (eds.), Brookfield, VT: A.A. Balkema, p. 115-122.

Analysis of extensive discontinuity characterization data collected from 3 field sites showed the joint locations to be spatially correlated. The degree of joint spatial correlation was determined through variogram calculations. Variograms were constructed assembling the data in 3 ways - joint spacing with respect to distance and with respect to joint sequence number, and joint trace density with respect to distance. The structured variograms suggested that the locations of joints within their rock masses were not purely random. Ranges of influence were found to be as large as $30-40 \mathrm{~m}$. The nature of the correlation could be modeled with spherical or modified hole effect variogram models. More realistic models of the rock mass can be achieved when joint spatial correlation is incorporated into rock mass simulations.

Young, D.S., 1987a, Random vectors and spatial analysis by geostatistics for geotechnical applications, Mathematical Geology, v. 19, no. 6, p. 467-479. 
Geostatistics is extended to the spatial analysis of vector variables by defining the estimation variance and vector variogram in terms of the magnitude of difference vectors. Many random variables in geotechnology are in vectorial terms rather than scalars, and its structural analysis requires those sample variable interpolations to construct and characterize structural models. A better local estimator will result in greater quality of input models; geostatistics can provide such estimators: kriging estimators. The efficiency of geostatistics for vector variables is demonstrated in a case study of rock joint orientations in geological formations. The positive crossvalidation encourages application of geostatistics to spatial analysis of random vectors in geoscience as well as various geotechnical fields including optimum site characterization, rock mechanics for mining and civil structures, cavability analysis, petroleum engineering, and hydrologic models.

Young, D.S., 1987b, Indicator kriging for unit vectors; rock joint orientations, Mathematical Geology, v. 19, no. 6, p. 481-501.

Indicator kriging (IK) is extended to analyze 3-D random unit vectors and evaluate the local probability distribution of rock joint orientations in geological formations. The pole vector representing joint orientations is regionalized and projected on a plane normal to the mean attitude of the joint family and centered at the mean. A 2$\mathrm{D}$ cutoff system is developed to define the indicator variable, and corresponding indicator variograms and indicator kriging. The cutoff system defines probability regions similar to those of a bivariate distribution, concentric rings sliced into radial sectors. A case study made on an open pit mine proved positively the efficiency of IK and encourages its application to localized probabilistic structural modeling for geotechnical or geohydrological analysis and oil/gas reservoir analysis.

Young, D.S. and S.F. Hoerger, 1988, Geostatistics applications to rock mechanics, Key Questions in Rock Mechanics, Proceedings of the 29th U.S. Symposium, June 13-15, 1988, Minneapolis, MN, P.A. Cundall, R.L. Sterling, and A.M. Starfield (eds.), Brookfield, VT: A.A. Balkema, p. 271-282.

A local probability of slope stability analysis was made for an open pit mine by introducing geostatistics into the key block theory. Indicator kriging was used to develop a localized probabilistic cell-block model of joint systems observed in the pit. Kinematic analysis of wedge failure was applied to the joint system model; a full probability of slope failure in each cell-block was obtained by stochastic simulation over random samples from Latin Hypercube sampling. Compared with the stochastic probability of slope failure and deterministic failure analysis based on the global sample data and its mean attitudes, respectively, the localized probability of failure marked a significant improvement in slope stability analysis. This is especially true when the local probability is obtained in the early stages of design and site exploration, when only sparse sample data is available. This approach is equally applicable to underground structures such as tunnels, storage caverns, and waste repositories 


\section{Appendix.2: Modeling of Jointed Rock}

Bandis, S.C., 1990, Scale effects in the strength and deformability of rocks and rock joints, Scale Effects in Rock Masses, Proceedings of the First International Workshop, June 7-8, 1990, Loen, Norway, A. Pinto Da Cunha (ed.), Brookfield, VT: A.A. Balkema, p. 59-76.

Blejwas, T.E. and F.D. Hansen, 1990, Scale effects in the shear behavior of joints in welded tuff, International Symposium on Rock Joints, June 4-6, 1990, Loen, Norway, N. Barton and O. Stephansson (eds.), Brookfield, VT: A.A. Balkema, p. 185-189.

Any opening instabilities in a possible nuclear waste repository at Yucca Mtn. are likely to be the result of movements along pre-existing joints in welded tuffs. Preliminary analyses suggest some potential for joint shear, but data are not available for evaluating the effect that large joint irregularities have on limiting displacements. Tests using sample sizes up to those that yield dilations and normal forces representative of in situ conditions are desirable for the proper characterization of shear behavior along irregular joints. A program of laboratory and field experiments will investigate the coupled effects of sample sizes and normal boundary conditions on shear behavior of joints in welded tuffs.

Cai, M. and H. Horii, 1993, A constitutive model and FEM analysis of jointed rock masses, International Journal of Rock Mechanics and Mining Sciences \& Geomechanics Abstracts, v. 30, no. 4, p. 351-359.

A constitutive model of jointed rock masses is presented which reflects the size, density, orientation, and connectivity of joints as well as their mechanical properties. Following the continuum approach, the incremental stress-strain relation of the jointed rock mass is formulated by taking the volume average of stress and strain inside a representative volume element where the evaluation of the relative displacement across the joints is required. Employing an elasto-plastic constitutive model of the joint behavior, the relative displacement across the joint can be obtained once the stress acting on the joint is known. The fundamental difficulty in the constitutive modeling of jointed rock masses is due to the fact that the stress acting on a joint is different from the avg. stress since a joint does not cut through the rock mass but terminates within the rock mass, possible connecting w/ other joints. The lower the stiffness of the surrounding matrix is, due to the existence of other joints of the connection of joints, the higher the stress acting on the joint will be. In the present study, the stress concentration tensor, which gives a relation between avg. stress and the stress acting on the joint, is introduced and a simple method to evaluate it is developed. The interaction effect between joints and the effect of joint connection are properly considered in the model. Some simple examples are solved by the proposed constitutive model. The results are in 
agreement with experimental data showing the characteristic features of the behavior of jointed rock masses. The proposed constitutive model for jointed rock masses is implemented into a finite element analysis program with a 3-D isoparametric element to analyze actual engineering problems. As an example, the program is used to analyze a plate-loading test problem and the results of the 3-D finite element analysis of the problem are compared w/ the test data. For highly jointed rock masses, the continuum model offers a powerful analytical method.

Detournay, E. and C. St. John, 1985, Equivalent continuum of large scale modeling for rock masses; an alternative approach, Research and Engineering Applications in Rock Masses, 26th U.S. Symposium on Rock Mechanics, June 26-28, 1985, Rapid City, SD, E. Ashworth (ed.), Boston, MA: A.A. Balkema, p. 131-138.

A representative element of some average dimension must be treated as a "continuum" and be assigned homogeneous stress-strain relations. The length of resolution, denoted here as gamma, is dependent upon the size and requirements of the problems modeled and in practice may correspond to the size of the smallest region represented in a numerical idealization; such as the smallest element in a FE model. Several empirical procedures for scaling the lab values of the elastic moduli and strength of the rock to in-situ values on the basis of quality indices, such as RQD, Q, or RMR have been proposed. However, these procedures are generally deficient because they fail to account for the resolution at which numerical modeling is performed. Accordingly, several investigators have developed semiempirical procedures in which the material properties of a given volume are related to the corresponding properties of a lab sample via relationships involving sample dimensions as well as quality. These have found most application in development of the properties of rock pillars, but are conceptually suited for numerical modeling, providing that the material properties of each zone in the model are adjusted in accordance with its dimensions. The alternatives to simple empirical procedures involve developing models that directly account for the presence of the fractures. Along those lines, equivalent anisotropic models and ubiquitous joint models have received much attention. These approaches have their limitations. A better, but often impractical approach, is to explicitly model each fracture. Here we propose a more practical alternative; one that involves two levels of definition of the rock mass. The first, or global level corresponds to the continuum representation at the scale of resolution we designated gamma. The second is a macroscopic level, at which the discontinuities and inhomogeneities of the rock can be treated.

Endo, H.K., J.C.S. Long, C.R. Wilson, and P.A. Witherspoon, 1984, A model for investigating mechanical transport in fracture networks, Water Resources Research, v. 20, no. 10, p. $1390-1400$.

A technique is presented to determine when anisotropic fracture systems can be modeled as equivalent porous media (continua) for transport. In order to use the continuum approach, one must demonstrate that the fracture system has the same 
transport behavior as an equivalent porous medium. Hydraulic effective porosity is calculated as the product of specific discharge and mean travel time, divided by linear length of travel. Specific discharge and hydraulic effective porosity are measured in different directions of flow in regions of varying size w/ constant hydraulic gradients. If the fracture system behaves like an equivalent porous medium, directional flow has the following properties: (1) specific discharge can be predicted from a permeability tensor and (3) hydraulic effective porosity is independent of direction of flow. A numerical model has been developed to simulate mechanical transport under steady flow in a discrete fracture network. The model is used to determine the distribution of travel times from inlet to outlet for fluid traveling in stream tubes. We have examined only systems $\mathrm{w} /$ parallel fracture sets in which all fractures are long compared to the region under study. These systems satisfy criterion 1 in that flux can be calculated using a porous medium equivalent. However, these systems do not satisfy criterion 2 because hydraulic effective porosity is shown to be directionally dependent. Thus, even though flux can be accurately predicted using porous medium assumptions for some fracture systems, it may not be possible to accurately predict mechanical transport using these same assumptions.

Grossmann, F., 1990, Correlation between the jointing index and the rock mass deformability, Scale Effects in Rock Masses, Proceedings of the First International Workshop, June 7-8, 1990, Loen, Norway, A. Pinto Da Cunha (ed.), Brookfield, VT: A.A. Balkema, p. 261-265.

If we assume that the different joint sets existing in a given rock mass may be modeled each as a Poisson process, and if we further accept that the rock mass deformability in a chosen direction depends linearly on the number of discontinuities occurring along that direction in the rock mass volume considered, it can be shown that, for the jointing indices usually found in practice, the REV is much larger than the volumes involved in classical in-situ deformability tests.

Grossmann, N.F., 1983, A numerical method for the definition of discontinuity sets, Fifth International Congress on Rock Mechanics, Melbourne, Australia, Boston, MA: A.A. Balkema, p. B17-B21.

This paper describes the numerical method developed at the LNEC which allows the complete determination of the existing discontinuity system starting from the total field data collection. The method may be applied to any number of the sets of information obtained and can also take into consideration cases of nonuniform sampling of the joints. Determination of the existing discontinuity sets is done through different steps, the 1st one using as a basis the spherical Poisson distribution and the other ones the bivariate normal distribution on the tangent plane at the mean attitude. 
Hoerger, S.F. and D.S. Young, 1987, Predicting local rock mass behavior using geostatistics, Rock Mechanics: Proceedings of the 28th U.S. Symposium, June 29-July 1, 1987, Tucson, AZ, I.W. Farmer, J.J.K. Daemen, C.S. Desai, C.E. Glass, and S.P. Neuman (eds.), Boston, MA: A.A. Balkema, p. 99-106.

Geostatistics can be used to make local estimates of spatially correlated rock mass properties. Local estimates, used as design inputs, allow a geotechnical design to make local adjustments to locally varying rock mass conditions. The use of geostatistics for local estimation of design inputs is illustrated using rock joint orientation data for slope stability analysis of a surface mine. The local inputs suggest local designs which are significantly different from the design made using site-wide averages composited from all sampled locations within the mine.

Hu, K.X. and Y. Huang, 1993, Estimation of the elastic properties of fractured rock masses, International Journal of Rock Mechanics and Mining Sciences \& Geomechanics Abstracts, v. 30, no. 4, p. 381-394.

The discontinuities in rock masses in the form of joints, fissures, and interface separations are crucial to the design of excavations. The effects of such discontinuities can often be characterized by the effective moduli of the fractures rock mass. In this paper, an estimation of the effective moduli is presented by modeling these discontinuities as planar tunnel cracks. The interaction among the cracks is accounted for within the framework of self-consistent mechanics. Three geometries -- randomly distributed cracks, parallel cracks, and two orthogonal sets of cracks -- are used to simulate the discontinuities in rock masses where all tunnel cracks are parallel to a certain direction. The uncracked material is assumed isotropic, while the cracked solid behaves as an orthotropic material. The damaged elastic moduli, in-plane and out-of-plane, are presented in terms of a defined planar crack density, which can be easily obtained from the geological cell statistics. A critical condition of crack densities at which the in-plane effective moduli vanish is established for each geometry. For all 3 cases, the out-of-plane moduli decay much slower than the in-plane moduli as the crack density increases. Also addressed in detail is the effect of the interaction between the 2 orthogonal sets of cracks on the damaged elastic properties. Some applications to geomechanics are discussed, and an investigation is made of the scale dependence of the modulus.

Kazi, A. and Z. Sen, 1985, Volumetric RQD: an index of rock quality, Proceedings of the International Symposium on Fundamentals of Rock Joints, September 15-20, 1985, Bjorkliden, Sweden, O. Stephansson (ed.), Lulea, Sweden: CENTEK Publ., p. 95102.

The use of a single value for the conventional RQD is generally insufficient to provide an adequate description of rock mass quality. A new rock mass quality parameter, volumetric RQD, which is not influenced by the direction in which the measurements are taken is proposed. To calculate the VRQD, the volumes of intact 
blocks gibber than $0.001 \mathrm{~m}^{\wedge} 3$ are summed and expressed as a percentage of the total rock mass volume. The upper and lower bounds for the VRQD in relation to volumetric fracture frequency and the number of matrix blocks per cubic meter have been defined deterministically. A method of counting the number of fractures in a unit volume of rock is included. This method is further applied for estimating the average volume of a matrix block. Using the negative exponential distribution, a relationship between the VRQD and frequency of matrix blocks is presented.

Kulatilake, P.H.S., 1988, State-of-the-art in stochastic joint geometry modeling, Key Questions in Rock Mechanics, Proceedings of the 29th U.S. Symposium, June 13-15, 1988, Minneapolis, MN, P.A. Cundall, R.L. Sterling, and A.M. Starfield (eds.), Brookfield, VT: A.A. Balkema, p. 215-229.

This paper surveys the published literature in stochastic joint geometric modeling. The review covers materials published through the middle of 1987 . It is mainly intended as a point of access to the literature. The paper also provides strengths and weaknesses of the available techniques to model joint geometrical parameters. The areas where future research efforts should be focused are summarized.

La Pointe, P.R., 1988, A method to characterize fracture density and connectivity through fractal geometry, International Journal of Rock Mechanics and Mining Sciences \& Geomechanics Abstracts, v. 25, no. 6, p. 421-429.

A method is presented to compute an index of fracture density using fractal geometry. Two alternative formulations are tested using computer-generated and natural rock fracture patterns. The first formulation examines the number of fractures per unit area of rock. The second is based on the density of fracturebounded blocks. The results show that fracture density, defined using either of these 2 formulations, is fractal and often scale-invariant. Computer simulations suggest that the number of fractures (or blocks), their size and size variability, and the number of fracture orientations affect the fractal dimension. The fractal dimension is most sensitive to the number of fractures or blocks, rather than their size, orientation, or size variability. This sensitivity to fracture or block density, coupled $w /$ the fact that the fractal dimension can be calculated w/out knowing fracture sizes, suggests that the method might provide a useful index of fracture intensity in the subsurface where data are obtained from core or boreholes. Moreover, the block density formulation may provide an index of fracture permeability, although this has not been tested.

Lin, D. and C. Fairhurst, 1991, The topological structure of fracture systems in rock, Rock Mechanics as a Multidisciplinary Science, Proceedings of the 32nd U.S. Symposium, July 10-12, 1991, Norman, OK, J-C. Roegiers (ed.), Brookfield, VT: A.A. Balkema, p. 1155-1163. 
Methods for analysis of the spatial structure of fracture systems, ranging from faults in rock masses to rock microstructures, are essential for predicting the geotechnical behaviors of rock. This paper outlines a method for extending the basic concepts and principles of combinatorial topology to the spatial analysis of 3$\mathrm{D}$ networks of discrete fractures. The fracture system is regarded as an algebraic structure formed by simplicial complexes. Fluid flow and transport through a fracture system is simulated, based on the topological structure of the fracture system and boundary integration of this structure.

Munier, R., 1993, Four-dimensional analysis of fracture arrays at the Aspo Hard Rock Laboratory, SE Sweden, Engineering Geology, v. 33, no. 3, p. 159-175.

During excavation and construction of Aspo HRL, geological information is systematically recorded and filed in a database called GEOTAB. Statistical analyses have been performed on fracture information from GEOTAB and stochastically chosen outcrops have been mapped in detail. Based on systematic recording of crosscutting relations, a succession of minerals and characteristics of decreasing age have been outlined. These include epidote, quartz, oxidized walls, iron oxides, chlorite, and calcite. The sequential fragmentation of the rock mass is described in terms of evolution of fracture arrays in time. A correction procedure for sampling biases has been performed and fracture traits have been analyzed. It is concluded that fracture lengths follow a lognormal distribution w/ mean, mode, and std dev estimated. Spacing has been evaluated for different fracture sets. It is concluded that the fracture array consists of six sets with different kinematics. Fracture zones underground are compared to a fracture zone at the surface.

Muralha, J., and A. Pinto Da Cunha, 1990a, Analysis of scale effects in joint mechanical behaviour, Scale Effects in Rock Masses, Proceedings of the First International Workshop, June 7-8, 1990, Loen, Norway, A. Pinto Da Cunha (ed.), Brookfield, VT: A.A. Balkema, p. 191-200.

A group of joints of a schistous rock mass, belonging to 3 joint sets, was submitted to lab shear tests. By cutting the samples parallel to the intial shear into 2,3 , and 4 parts and re-shearing them, the influence of test size on the mechanical properties of rock joints was done.

Muralha, J., and A. Pinto Da Cunha, 1990b, About LNEC experience on scale effects in the mechanical behaviour of joints, Scale Effects in Rock Masses, Proceedings of the First International Workshop, June 7-8, 1990, Loen, Norway, A. Pinto Da Cunha (ed.), Brookfield, VT: A.A. Balkema, p. 131-148.

The results of a certain number of laboratory and in situ shear tests carried out by LNEC for several hydroelectrical projects have been reviewed, and the published and unpublished data has been re-analyzed from the point of view of the influence of sample dimensions on the deformability 
and strength characteristics of the joints.

The research was carried out mainly in terms of the avg. properties of systematic joint sets and the scattering of such properties, for a given site. In some cases, different joint types were defined, based on lithology, roughness, and filling characteristics and analysis was made of the variation of the joint mechanical properties $w /$ the size of the shear sample.

Pinnaduwa, H.S.W., N.W. Deepa, and O. Stephansson, 1990, Three dimensional stochastic joint geometry modeling including a verification; a case study, Rock Mechanics:

Contributions and Challenges, Proceedings of the 31st U.S. Symposium, June 1820, 1990, Golden, CO, W.A. Hustrulid and G.A. Johnson (eds.), Brookfield, VT: A.A. Balkema, p. 1051-1058.

Since joint geometry patterns can vary from one region to another, each region should be represented by a separate joint geometry model. Therefore, the first step should be identification of statistically homogeneous regions. It then is necessary to know the number of joint sets, and for each joint set, the intensity, spacing, location, orientation, shape, and dimension distributions. These joint parameters are inherently statistical. Sample values of joint parameters provided by the field data usually are subject to sampling biases and represent only 1- or 2-D properties. Therefore, before inferring these parameters from sampling values, field data should be corrected for sampling biases. In addition, principles of stereology must be used to infer 3D parameters from 1- or 2-D values. At present, a 3-D joint geometry modeling scheme which incorporates these features and also includes a formal verification procedure is not avail in the literature. This paper shows the development of 3-D joint geometry models which investigate statistical homogeneity, include correction for sampling biases, and applications of stereological principles. It also checks the validity of such models (?).

Sage, J.D., A.A. Aziz, and E.R. Danek, 1990, Aspects of scale effects on rock closure, Scale Effects in Rock Masses, Proceedings of the First International Workshop, June 7-8, 1990, Loen, Norway, A. Pinto Da Cunha (ed.), Brookfield, VT: A.A. Balkema, p. 175-181.

Difficulties in predicting the load-deformation behavior of jointed rock which is based on lab or field studies on small samples are first described. Theoretical studies of rock opening and closure based on an analysis of joint surface topography are presented which suggest a scaling factor based on sample size and deformation history of the insitu jointed rock mass. The data from lab loaddeformation studies of rock closure on models of jointed rock specimens ranging in size from $100-900 \mathrm{~cm}^{2}$ coupled $\mathrm{w} /$ the results of profilometer tests on the joint surfaces and finite element analysis is presented in support of theoretical considerations. 
Sarra Pistone, R., 1990, Scale effect in the shear strength of rock joints, Scale Effects in Rock Masses, Proceedings of the First International Workshop, June 7-8, 1990, Loen, Norway, A. Pinto Da Cunha (ed.), Brookfield, VT: A.A. Balkema, p. 201-206.

In rock mechanics the representativeness of lab test data is a fundamental issue. Systematic tests on plaster samples w/ different dimensions and surfaces roughnesses were carried out by Bandis, showing a remarkable diminution in shear strength $w /$ increasing sample size. That same data were used here to establish an extrapolation model from lab data to natural block dimensions. The roughness profiles were digitized and a theoretical model was developed that used JRC (Barton) as the controlling parameter. "Slope diagrams" were made and a classification of discontinuity characterization was proposed. Case studies then were done, providing a good correlation between extrapolation model prediction and theoretical predictions.

Sen, Z., 1990a, Cumulative core index for rock quality evaluations, International Journal of Rock Mechanics and Mining Sciences \& Geomechanics Abstracts, v. 27, no. 2, p. 87-94.

The cumulative core index is a numerical core logging procedure proposed for evaluating the rock quality in drill cores. It provides an objective, convenient, and rapid means of quantifying a fundamental rock mass property. This technique takes into account 3 potential weakness sources in a rock mass: the natural joints, core fragments cut by these joints (broken core zones), and the core losses. The cumulative measurements of these variables along the borehole length lead to the cumulative core index as the summation of source percentages $\mathrm{w} /$ special weights given to joints which separate the intact core lengths that are greater than a specified threshold level. Any increase in the discontinuity number increases the cumulative core index, implying deterioration in rock quality. In deciding the values of weakness weights of joints, the orientation angle of the joints wrt the core axis are taken into consideration. The necessary expressions that relate the cumulative core index to the classical core index and RQD are presented on the basis of a negative exponential distribution for the joint spacings. Practical charts are developed for the relation between the cumulative core index and the average joint number. Furthermore, a complete discussion is provided for comparison of the cumulative core indices w/ the RQD. Finally, the applications of the cumulative core index are presented for the discontinuities in granitic rocks and ash-fall tuffs.

Sen, Z., 1990b, RQP, RQR and fracture spacing, International Journal of Rock Mechanics and Mining Sciences \& Geomechanics Abstracts, v. 27, no. 2, p. 135-137. 
Sen, Z., 1992, Fractal dimension and rock quality charts, Bulletin of the Association of: Engineering Geologists, v. 29, no. 1, p. 77-85.

Fractal dimension is proposed as a useful parameter for characterizing the irregular fragmentation within a rock mass leading to a new empirical rock quality chart and classification. A new algorithm is developed which can be easily implemented provided that intact length measurements are avail along a borehole core or scanline on fresh cut rock surfaces. During the application of the algorithm, total recoverable intact length and their number are calculated for a given set of reference lengths. In general, the total recoverable intact length decreases as longer reference lengths are considered and their plot on arithmetic paper yields a line with negative slope. This plot is referred to as the Rock Quality Chart (RQC) and the slope of the regression straight line is adopted as the rock quality index. The smaller the slope, the better becomes the rock quality. It also is possible to obtain probable maximum block sized from a relevant RQC. On the other hand, the plot of reference lengths versus their number along a scanline also yields a straight line but on log-log paper with negative slope (S). Accordingly, the fractal dimension (D) for the scanline concerned is defined as 1-S. The significance of the fractal dimension in rock quality descriptions is explained in detail. Both concepts of rock quality charts and fractal dimensions are applied to some scanline measurements already avail in the literature.

Shi, G-H., R.E. Goodman, and J.P. Tinucci, 1985, Application of block theory to simulated joint trace maps, Proceedings of the International Symposium on Fundamentals of Rock Joints, September 15-20, 1985, Bjorkliden, Sweden, O. Stephansson (ed.), Lulea, Sweden: CENTEK Publ., p. 367-383.

Gathering rock mass data for surface excavations and underground openings is receiving more and more emphasis as the projects become larger and the associated risks increase. Yet there is a need to link the use of this data to existing analytical techniques. This paper describes a procedure by which field recorded discontinuity trace data can be used to identify discrete blocks and regions of maximum nested key blocks. A simple 2-D trace map simulation model is presented that is based on the superposition of 1-D strips. The principles of block theory are used to describe general block shapes and requires the identification of boundaries formed by the intersection of joints. Three examples are presented.

Villaescusa, E. and E.T. Brown, 1990, Characterizing joint spatial correlation using geostatistical methods, International Symposium on Rock Joints, June 4-6, 1990, Loen, Norway, N. Barton and O. Stephansson (eds.), Brookfield, VT: A.A. Balkema, p. 115-122.

Analysis of extensive discontinuity characterization data collected from 3 field sites showed the joint locations to be spatially correlated. The degree of joint spatial correlation was determined through variogram calculations. Variograms were constructed assembling the data in 3 ways - joint spacing wrt distance and wrt 
joint sequence number, and joint trace density wrt distance. The structured variograms suggested that the locations of joints within their rock masses were not purely random. Ranges of influence were found to be as large as $30-40 \mathrm{~m}$. The nature of the correlation could be modeled $\mathrm{w} /$ spherical or modified hole effect variogram models. More realistic models of the rock mass can be achieved when joint spatial correlation is incorporated into rock mass simulations.

Wu, F., 1988, A 3d model of a joint rock mass and its deformation properties, International Journal of Rock Mechanics and Mining Sciences \& Geomechanics Abstracts, v. 6, no. 2, p. 169-177.

Young, D.S., 1987b, Indicator kriging for unit vectors; rock joint orientations, Mathematical Geology, v. 19, no. 6, p. 481-501.

Indicator kriging (IK) is extended to analyze 3-D random unit vectors and evaluate the local probability distribution of rock joint orientations in geological formations. The pole vector representing joint orientations is regionalized and projected on a plane normal to the mean attitude of the joint family and centered at the mean. A 2-D cutoff system is developed to define the indicator variable, and corresponding indicator variograms and indicator kriging. The cutoff system defines probability regions similar to those of a bivariate distribution, concentric rings sliced into radial sectors. A case study made on an open pit mine proved positively the efficiency of IK and encourages its application to localized probabilistic structural modeling for geotechnical or geohydrological analysis and oil/gas reservoir analysis.

Zhang X., 1990, Estimation of geometrical parameters of key discontinuities within an engineering rock region, International Journal for Numerical and Analytical Methods in Geomechanics, v. 14, no. 5, p. 367-377.

Discontinuities that have unfavorable orientation and are continuous within overall engineering rock regions can have a dominant effect on the strength, deformability, and permeability of the rock mass. The concepts of geometrical parameters of basic discontinuities and engineering discontinuities are proposed in this communication. Further, the engineering discontinuities are divided into key discontinuities and non-key disc's. Within any region of the rock mass, the spacing, trace length, and probability of engineering discontinuities can be estimated from the geometrical parameters of the basic discontinuities. In general, the geometrical parameters are different from those of the basic discontinuities. Finally, two examples are given to illustrate how to apply these parameters to rock engineering problems. 


\section{Appendix.3: Indirect or Inverse Approaches}

Hoeksema, R.J. and P.K. Kitanidis, 1984, An application of the geostatistical approach to the inverse problem in two-dimensional groundwater modeling, Water Resources Research, v. 20, no. 7, p. 1003-1020.

Long, J.C.S., K. Karasaki, A. Davey, J. Peterson, M. Landsfeld, J. Kemeny, and S. Martel, 1991, An inverse approach to the construction of fracture hydrology models conditioned by geophysical data: an example from the validation exercises at the Stripa Mine, International Journal of Rock Mechanics and Mining Sciences \& Geomechanics Abstracts, v. 28, no. 2/3, p. 121-142.

Moris, J.P.E. and K.I. Oravecz, 1985, Theoretical background to the development of a computer code for the modeling of seam-like deposits, Research and Engineering Applications in Rock Masses, 26th U.S. Symposium on Rock Mechanics, June 2628, 1985, Rapid City, SD, E. Ashworth (ed.), Boston, MA: A.A. Balkema, p. 927936.

Panek, L.A., 1994, Scaling mine pillar size and shape with the Psi function, Mining Engineering, v. 46 , no. 11 , p. $1277-1281$.

A new pillar strength formula is presented, incorporating a function of size and shape, which takes into account the size-shape interaction effect as well as the enhanced strength exhibited by wide pillars. Existing pillar formulas, lacking these capabilities, are not suitable for extrapolating small-specimen test results to minepillar sizes and shapes. Interpreting compressive strength test results as a function of gamma clearly reveals wide-pillar behavior in much old test data. Model relationships are discussed, along with their scaling implications.

Rajaram, H. and D.B. McLaughlin, 1990, Identification of large-scale spatial trends in hydrologic data, Water Resources Research, v. 26, no. 10, p. 2411-2423.

It is often useful to distinguish different scales of variability in hydrologic properties such as hydraulic conductivity. In the simplest 2-scale case, large-scale variations can be viewed as a trend, while small-scale fluctuations about this trend can be viewed as a random residual. This paper describes a method for estimating spatial trends from scattered field measurements. The basic concept is to treat both the trend and the residual as stationary random functions. These functions are distinguished by their spatial spectral (or covariance) properties, which may be estimated from available data or simply hypothesized. We present 2 versions of a general algorithm for estimating spatial trends: (1) a discrete version that is useful in practical applications where data are limited and irregularly spaced and (2) a continuous version that can be used to study the effects of using incorrect spectral parameters. Applications of the discrete algorithm to both synthetically generated data and field measurements yield satisfactory trend estimates. An analysis based 
on the continuous algorithm shows that the estimation error lower bound for these applications depends on 2 dimensionless ratios: the scale disparity (ratio of the trend and residual correlation scales) and the signal-to-noise ratio (ratio of the trend and residual variances). These ratios may be used to evaluate the feasibility of trend estimation before field samples are actually collected.

\section{Appendix.4: Hydrological Properties}

Carlsson, A., G. Gustafson, U. Lindblom, and T. Olsson, 1990, Scale effects in the determination of hydraulic properties of rock masses, Scale Effects in Rock Masses, Proceedings of the First International Workshop, June 7-8, 1990, Loen, Norway, A. Pinto Da Cunha (ed.), Brookfield, VT: A.A. Balkema, p. 103-117.

Neuman, S.P., 1990, Universal scaling of hydraulic conductivities and dispersitivies in geologic media, Water Resources Research, v. 26, no. 8, p. 1749-1758.

An interpretation is offered for the observation that dispersivities increase w/ scale. Apparent longitudinal dispersivity data from a variety of hydrogeologic settings are assumed to represent a continuous hierarchy of $\log$ hydraulic conductivity fields $\mathrm{w} /$ mutually uncorrelated increments, each field having its own exponential autocovariance, associated integral scale, and variance that increases as a power of scale. Such a hierarchy is shown theoretically to form a self-similar random field $w /$ homogeneous increments. Regardless of whether or not the underlying assumption is valid, one can justify interpreting the apparent dispersivities in a manner consistent $w /$ a recent quasi-linear theory of non-Fickian and Fickian dispersion in homogeneous media which supports the notion of a selfsimilar hierarchy a posteriori. The hierarchy is revealed to possess a semivariogram $\operatorname{gamma}(\mathrm{s})=\mathrm{cs}^{\wedge} 0.5$, where $\mathrm{c}$ is a constant, and a fractal dimension $\mathrm{D}=\mathrm{E}-0.75$, where $E$ is the topological dimension of interest. This can be viewed as a universal scaling rule about which large deviations occur due to local influences including the existence of discrete natural scales at which log hydraulic conductivity is statistically homogeneous. As such homogeneity is at best a local phenomenon occurring intermittently over narrow bands of the scale spectrum, one must question the utility of associating medium properties with representative elementary volumes and relying on Fickian models of dispersion over more than relatively narrow scale intervals. Porous and fractured media appear to follow the same idealized scaling rule for both flow and transport, raising a question about the validity of many distinctions commonly drawn between such media. Finally, the data suggest that conditioning transport models through calibration against hydraulic measurements has the effect of filtering out large-scale modes from the hierarchy. 
Oda, M. and Y. Hatsuyama, 1985, Permeability tensor for jointed rock masses, Proceedings of the International Symposium on Fundamentals of Rock Joints, September 15-20, 1985 , Bjorkliden, Sweden, O. Stephansson (ed.), Lulea, Sweden: CENTEK Publ., p. 303312.

Rock masses, which commonly contain a large number of discontinuities, are treated as homogeneous, anisotropic porous media in order to formulate the corresponding permeability tensor. This has been successfully achieved by introducing a symmetric tensor (crack tensor) that depends only on the geometry of the related cracks (aperture, size, and orientation). The principal directions associated with the symmetric crack tensor are coaxial with those of the permeability tensor. The first invariant of the crack tensor is proportional to the mean permeability, while the deviatoric portion is related to the anisotropic permeability. These results are well supported by numerical experiments on the permeability of cracked media by Long, Remer, Wilson, and Witherspoon (1982).

Phillips, F.M. and J.L. Wilson, 1989, An approach to estimating hydraulic conductivity spatial correlation scales using geological characteristics, Water Resources Research, v. 25 , no. 1 , p. 141-143.

Rajaram, H. and D.B. McLaughlin, 1990, Identification of large-scale spatial trends in hydrologic data, Water Resources Research, v. 26, no. 10, p. 2411-2423.

It is often useful to distinguish different scales of variability in hydrologic properties such as hydraulic conductivity. In the simplest 2-scale case, large-scale variations can be viewed as a trend, while small-scale fluctuations about this trend can be viewed as a random residual. This paper describes a method for estimating spatial trends from scattered field measurements. The basic concept is to treat both the trend and the residual as stationary random functions. These functions are distinguished by their spatial spectral (or covariance) properties, which may be estimated from available data or simply hypothesized. We present 2 versions of a general algorithm for estimating spatial trends: (1) a discrete version that is useful in practical applications where data are limited and irregularly spaced and (2) a continuous version that can be used to study the effects of using incorrect spectral parameters. Applications of the discrete algorithm to both synthetically generated data and field measurements yield satisfactory trend estimates. An analysis based on the continuous algorithm shows that the estimation error lower bound for these applications depends on 2 dimensionless ratios: the scale disparity (ratio of the trend and residual correlation scales) and the signal-to-noise ratio (ratio of the trend and residual variances). These ratios may be used to evaluate the feasibility of trend estimation before field samples are actually collected. 


\section{Appendix.5: Elastic Properties, Thermal Conductivity, and Strength Properties}

Bandis, S.C., 1990, Scale effects in the strength and deformability of rocks and rock joints, Scale Effects in Rock Masses, Proceedings of the First International Workshop, June 7-8, 1990, Loen, Norway, A. Pinto Da Cunha (ed.), Brookfield, VT: A.A. Balkema, p. $59-76$.

Bauer, S.J., R.K. Thomas, and L.M. Ford, 1985, Measurement and calculation of the mechanical response of a highly fractured rock, Research and Engineering Applications in Rock Masses, 26th U.S. Symposium on Rock Mechanics, June 26-28, 1985, Rapid City, SD, E. Ashworth (ed.), Boston, MA: A.A. Balkema, p. 523-530.

We have completed the first steps in attempting to validate a material constitutive model for a jointed rock mass. The continuum model, as utilized here within a finite element code, consists of a material constitutive description based on the linear elastic behavior of the matrix material and nonlinear normal and shear behavior of fracture planes. The validation exercise consists of (1) characterizing an appropriate physical model, (2) measuring the mechanical response of that physical model, and (3) using the physical model characteristics to calculate the mechanical response of the physical model. Calculated and measured responses for small-scale physical models (thermally-fractured granite) are in qualitative agreement. For deviatoric loadings, the calculated tangent modulus is an order of magnitude less than that of the matrix and exhibits stiffening with increasing load, and thereby functionally tracks the measured response

Blejwas, T.E. and F.D. Hansen, 1990, Scale effects in the shear behavior of joints in welded tuff, International Symposium on Rock Joints, June 4-6, 1990, Loen, Norway, N. Barton and O. Stephansson (eds.), Brookfield, VT: A.A. Balkema, p. 185-189.

Any opening instabilities in a possible nuclear waste repository at Yucca Mtn. are likely to be the result of movements along pre-existing joints in welded tuffs. Preliminary analyses suggest some potential for joint shear, but data are not available for evaluating the effect that large joint irregularities have on limiting displacements. Tests using sample sizes up to those that yield dilations and normal forces representative of in situ conditions are desirable for the proper characterization of shear behavior along irregular joints. A program of laboratory and field experiments will investigate the coupled effects of sample sizes and normal boundary conditions on shear behavior of joints in welded tuffs. 
Cuisiat, F.D. and B.C. Haimson, 1992, Scale effects in rock mass stress measurements, International Journal of Rock Mechanics and Mining Sciences \& Geomechanics Abstracts, v. 29, no. 2, p. 99-117.

The current state of knowledge on scale effects in rock stress measurements is summarized in this paper. One type of scale effect is inherent in the rock structure due to heterogeneities ranging from micro- to macro-scale. A second scale effect is involved through the scale of application considered. The implications of these scale effects are discussed. Emphasis is placed on consequences for stress measurement design.

Enever, J.R., R.J. Walton, and M.B. Wold, 1990, Scale effects influencing hydraulic fracture and overcoring stress measurements, Scale Effects in Rock Masses, Proceedings of the First International Workshop, June 7-8, 1990, Loen, Norway, A. Pinto Da Cunha (ed.), Brookfield, VT: A.A. Balkema, p. 317-326.

Results from many surface and sub-surface stress measurements by hydraulic fracturing and overcoring, in a variety of geological conditions, are exemplified in terms of the scale of the geologic structure, ranging from regional to local. Examples of close local and microscopic scale influences on the measurement techniques are also discussed. It is evident that there is an interaction between structure and stress across the range of scales, to which variability in stress measurement results can generally be related.

Fadeev, A.B., 1990, Scale effect of rock strength, Scale Effects in Rock Masses, Proceedings of the First International Workshop, June 7-8, 1990, Loen, Norway, A. Pinto Da Cunha (ed.), Brookfield, VT: A.A. Balkema, p. 183-189.

The scale effect in rock mechanics reveals an essential dependence of rock mechanical properties on the size of the tested sample. The practical scale effect of strength is predetermined by 3 mechanisms: damage during sampling, statistical law of behavior of "weakest link", and change of structural block stability due to increase of loaded zone.

Frappa, M., M. Rouai, and P. Morlier, 1990, Dynamic stiffness of a rock mass; scale effect, Scale Effects in Rock Masses, Proceedings of the First International Workshop, June 7-8, 1990, Loen, Norway, A. Pinto Da Cunha (ed.), Brookfield, VT: A.A. Balkema, p. 245-251.

It was possible to make numerous measurements of sound velocity in a carefully chosen quarry with different technologies. The relation between the mean value of sound velocity and log of wavelength is interpreted as a scale effect. A jump occurs when the wavelength is the same order of magnitude as structural characteristic dimensions of the rock mass. 
Grossmann, F., 1990, Correlation between the jointing index and the rock mass deformability, Scale Effects in Rock Masses, Proceedings of the First International Workshop, June 7-8, 1990, Loen, Norway, A. Pinto Da Cunha (ed.), Brookfield, VT: A.A. Balkema, p. 261-265.

If we assume that the different joint sets existing in a given rock mass may be modeled each as a Poisson process, and if we further accept that the rock mass deformability in a chosen direction depends linearly on the number of discontinuities occurring along that direction in the rock mass volume considered, it can be shown that, for the jointing indices usually found in practice, the REV is much larger than the volumes involved in classical in-situ deformability tests.

Gunsallus, K.L. and F.H. Kulhawy, 1984, Comparative evaluation of rock strength measures, International Journal of Rock Mechanics and Mining Sciences \& Geomechanics Abstracts, v. 21, no. 5, p. 233-248.

The variation in strength is investigated for 8 lithologically similar Silurian sedimentary rock units from NE U.S. This investigation included the fracture toughness, uniaxial compression, point load index, and Brazil tensile tests. The results show that the amount of variation associated w/ each test and formation is similar, although fracture toughness appears more consistent. The strength and in situ location correlations indicate that the variation in strength within a unit is of the same order of magnitude as the variation associated w/ each test. Existing correlations among the tests are examined in the framework of the data, and new correlations for predicting fracture toughness are presented. The variation in strength with saturation for sandstone also was examined; generally, strength decreases with saturation. Lastly, the variation in strength as a function of lithological differences within one formation is evaluated. The results indicate that it may be more important to sample a rock unit selectively in a number of different locations than to do extensive testing in one location.

Haimson, B.C., 1990, Scale effects in rock stress measurements, Scale Effects in Rock Masses, Proceedings of the First International Workshop, June 7-8, 1990, Loen, Norway, A. Pinto Da Cunha (ed.), Brookfield, VT: A.A. Balkema, p. 89-101.

Hu, K.X. and Y. Huang, 1993, Estimation of the elastic properties of fractured rock masses, International Journal of Rock Mechanics and Mining Sciences \& Geomechanics Abstracts, v. 30, no. 4, p. 381-394.

The discontinuities in rock masses in the form of joints, fissures, and interface separations are crucial to the design of excavations. The effects of such discontinuities can often be characterized by the effective moduli of the fractures rock mass. In this paper, an estimation of the effective moduli is presented by modeling these discontinuities as planar tunnel cracks. The interaction among the cracks is accounted for within the framework of self-consistent mechanics. Three 
geometries -- randomly distributed cracks, parallel cracks, and two orthogonal sets of cracks -- are used to simulate the discontinuities in rock masses where all tunnel cracks are parallel to a certain direction. The uncracked material is assumed isotropic, while the cracked solid behaves as an orthotropic material. The damaged elastic moduli, in-plane and out-of-plane, are presented in terms of a defined planar crack density, which can be easily obtained from the geological cell statistics. A critical condition of crack densities at which the in-plane effective moduli vanish is established for each geometry. For all 3 cases, the out-of-plane moduli decay much slower than the in-plane moduli as the crack density increases. Also addressed in detail is the effect of the interaction between the 2 orthogonal sets of cracks on the damaged elastic properties. Some applications to geomechanics are discussed, and an investigation is made of the scale dependence of the modulus.

Martin, C.D., R.S. Read, and N.A. Chandler, 1990, Does scale influence in situ stress measurements? Some findings at the underground research laboratory, Scale Effects in Rock Masses, Proceedings of the First International Workshop, June 7-8, 1990, Loen, Norway, A. Pinto Da Cunha (ed.), Brookfield, VT: A.A. Balkema, p. 307316.

Miller, S.M. and R.D. Luark, 1993, Spatial simulation of rock strength properties using a MarkovBayes method, International Journal of Rock Mechanics and Mining Sciences \& Geomechanics Abstracts, v. 30, no. 7, p. 1631-1637.

In most rock engineering projects, actual measurements of rock strengths often are limited to a few observations over large volumes of the rock mass. However, these few data can be complemented by secondary information such as qualitative lithology descriptions or drilling characteristics, which typically are available across much of the study region. A Markov-Bayes method can be used to combine these types of information to produce spatial simulations of rock strength properties. A case study that illustrates this simulation procedure has been completed for the siltstone roof in a selected $6 \times 18 \mathrm{~m}$ area of an underground coal mine. Threedimensional simulations, or stochastic images, of the uniaxial compressive strength and the modulus of elasticity were generated using hard data from 4 core holes and secondary data in the form of averaged specific energy of drilling values from 95 rockbolt holes. Such images not only provide a probabilistic model of rock strength, but also describe the natural variability and spatial fluctuations as well.

Morgan, H.S. and R.D. Krieg, 1990, Investigation of an empirical creep law for rock salt that uses reduced elastic moduli, Rock Mechanics: Contributions and Challenges, Proceedings of the 31st U.S. Symposium, June 18-20, 1990, Golden, CO, W.A. Hustrulid and G.A. Johnson (eds.), Brookfield, VT: A.A. Balkema, p. 965-972.

This paper presents the results of shaft calculations to investigate why an empirical fix for WIPP rock mass models (divide $\mathrm{E}$ by 12.5 ) has worked so well in other areas of the WIPP site. 
Natau, O., 1990, Scale effects in the determination of the deformability and strength of rock masses, Scale Effects in Rock Masses, Proceedings of the First International Workshop, June 7-8, 1990, Loen, Norway, A. Pinto Da Cunha (ed.), Brookfield, VT: A.A. Balkema, p. 77-88.

Ode, M., T. Yamabe, Y. Ishizuka, H. Kumasaka, H. Tada, and K. Kimura, 1991, Elastic stress and strain jointed rock masses by means of crack tensor analysis, Rock Mechanics and Rock Engineering, v. 26, no. 2, p. 89-112.

An elastic stress-strain relation is formulated in terms of crack tensors which makes it possible to take into account explicitly the effect of joints on the elastic behavior of rock masses. The present study is to discuss some related topics which may be encountered in its practical application. Two problems are solved by incorporating the elastic stress-strain relation into a program for 3-D finite element analyses; i.e., stress concentration by surface loading and displacement by excavation of an intersecting tunnel. Validity of the results is checked by comparing them with a lab model test and a field test, with the following conclusions: The overall distribution of stress definitely depends on a joint stiffness ratio (i.e., normal stiffness to shear stiffness). If the ratio is chosen as unity, the stress concentration occurs mainly in the direction parallel to major joints. If the ratio is high, say 10 , then the stress concentrates along the perpendicular as well as the parallel directions to major joints. It can be said, on the basis of the fairly good agreement of the calculations using the high stiffness ratio with the field and lab measurements, that the elastic solution by crack tensor provides a practical tool for estimating the stress and strain in strongly jointed rock masses.

Pariseau, W.G. and H. Moon, 1988, Elastic moduli of well-jointed rock masses, Proceedings of the Sixth International Conference on Numerical Methods in Geomechanics, April 11-15, 1988, Innsbruck, Austria, G. Swoboda (ed.), Brookfield, VT: A.A. Balkema for the International Committee on Numerical Methods in Geomechanics, p. 815822.

A theoretical approach to the description of the overall elastic properties of well-jointed rock masses is outlined. The approach is based on a sequential application of 2-phase composite theory of linearly elastic media. The finite element method is used in numerical experiments to obtain data from which the true overall properties are calculated. Example results involving jointed and faulted rock masses show excellent agreement between theory and numerical experiment. The technique is confirmed by 2 entirely different programming \& testing efforts that give the same results for equal rock and joint Poisson's ratios. 
Pariseau, W.G., 1993, Equivalent properties of jointed Biot material, International Journal of Rock Mechanics and Mining Sciences \& Geomechanics Abstracts, v. 30, no. 7, p. 1151-1157.

In many practical cases, rock mass structural and material discontinuities are too numerous to be accounted for on a one-to-one basis, so that an equivalent properties approach is necessary. All existing equivalent properties formulations are based on the assumption of an REV that severely restricts application to engineering design. Application of a non-REV procedure to coupled, poroelastic constitutive equations is described here. The results are in excellent agreement with constraints intrinsic to Biot's material law and with previous non-REV results obtained in application to Hooke's law in linear elasticity and to Darcy's law in seepage analysis. Results for the classic 1-D consolidation problem of a layered isotropic material are in reasonable agreement w/ results for the same sample characterized by equivalent homogeneous, anisotropic properties.

Peres-Rodrigues, F., 1990, About LNEC experience on scale effects in the deformability of rocks, Scale Effects in Rocks, Proceedings of the First International Workshop, June 7-8, 1990, Loen, Norway, A. Pinto Da Cunha (ed.), Brookfield, VT: A.A. Balkema, p. 155-164.

In simple compression tests on 3 types of rocks 35,600 determinations were carried out which have made it possible to develop the following conclusions:

- The values of strains measured on each rock type follow a normal distribution and thus the mean value of the corresponding deformability modulus is the harmonic mean. I.e., deformability modulus depends only on joint frequency.

- The mean values of the deformability modulus of a rock mass is independent of the test area and of the gage length, with the assumptions that the rock mass is homogeneous, divided by a system of joints normal to the force, and with constant joint intensity. Corresponding std dev's decrease as the gage length increases, asymptotically tending to zero.

- Safety against deformation increases w/ the foundation area, for structures that convey identical stresses to the same rock mass. Thus it will be possible to make savings in those works within the present safety criteria.

Pinto Da Cunha, A. and J. Muralha, 1990, About LNEC experience on scale effects in the deformability of rock masses, Scale Effects in Rock Masses, Proceedings of the First International Workshop, June 7-8, 1990, Loen, Norway, A. Pinto Da Cunha (ed.), Brookfield, VT: A.A. Balkema, p. 219-229.

A review of several deformability studies of rock masses carried out by LNEC, with a reanalysis and a reinterpretation of existent data is presented, emphasizing the variation of the rock mass deformability moduli w/ the tested volumes. Since different laboratory and in situ tests were considered for each site, an attempt to understand the associated scale effects is made, taking into due consideration the 
influence of distinct testing techniques and potential sampling bias in the variation and scattering of the test results.

Ratigan, J.L., 1990, Scale effect in the hydraulic fracture test associated with the estimation of tensile strength, Scale Effects in Rock Masses, Proceedings of the First International Workshop, June 7-8, 1990, Loen, Norway, A. Pinto Da Cunha (ed.), Brookfield, VT: A.A. Balkema, p. 297-306.

Application of classic hydraulic fracture theory results in the measurement of two quantities: the breakdown pressure and the instantaneous shut-in pressure. However, a third quantity is necessary to evaluate the in situ stresses -- the hydraulic fracture tensile strength of the rock. Several methods have been proposed to estimate this quantity. Some of the methods require laboratory testing while others require additional testing in the field. A method for estimating the field hydraulic fracture tensile strength originally developed by Weibull is presented. Using this statistical fracture mechanics method, laboratory hydraulic fracture tests or laboratory direct tension tests can be used in the interpretation of field hydraulic fracture tests. Such as application can minimize potential scale effects. An example based on a field test in the Stripa Mine in Sweden is presented.

Sage, J.D., A.A. Aziz, and E.R. Danek, 1990, Aspects of scale effects on rock closure, Scale Effects in Rock Masses, Proceedings of the First International Workshop, June 7-8, 1990, Loen, Norway, A. Pinto Da Cunha (ed.), Brookfield, VT: A.A. Balkema, p. 175-181.

Difficulties in predicting the load-deformation behavior of jointed rock which is based on lab or field studies on small samples are first described. Theoretical studies of rock opening and closure based on an analysis of joint surface topography are presented which suggest a scaling factor based on sample size and deformation history of the insitu jointed rock mass. The data from lab loaddeformation studies of rock closure on models of jointed rock specimens ranging in size from $100-900 \mathrm{~cm}^{2}$ coupled $\mathrm{w} /$ the results of profilometer tests on the joint surfaces and finite element analysis is presented in support of theoretical considerations.

Uhle, R.J., Jr. and D. Van Zyl, 1990, Shear strength and deformation parameters of rockfill related to particle size, Rock Mechanics: Contributions and Challenges, Proceedings of the 31 st U.S. Symposium, June 18-20, 1990, Golden, CO, W.A. Hustrulid and G.A. Johnson (eds.), Brookfield, VT: A.A. Balkema, p. 981-988.

This paper's purpose is to review the available published rockfill data and to evaluate, through statistical analyses, the relationships of shear strength and deformation parameters with particle size. The data were used further to statistically investigate the effects of other influencing factors such as rock type, 
confining stress, and saturation on the shear strength and deformation parameters of rockfill.

van Stone, L.J., 1985, A Monte Carlo method for studying thermal conductivity and thermal anisotropy in aggregates, Research and Engineering Applications in Rock Masses, 26th U.S. Symposium on Rock Mechanics, June 26-28, 1985, Rapid City, SD, E. Ashworth (ed.), Boston, MA: A.A. Balkema, p. 917-923.

Monte Carlo methods allow simulation of systems having uncontrolled parameters. Many rock units have quite variable compositions, and even those which are very uniform have very little regularity on a fine scale. Therefore, methods of estimating bulk properties derived using periodic or regularized geometries are not very successful. The Monte Carlo method described here allows the introduction of specific amounts of randomness in order to learn the scatter inherent in certain types of constrained-random systems. This work is related to studies of thermal anisotropy in rocks being pursued at South Dakota School of Mines and Technology. Computer memory limitations and time constraints made the use of 3-D finite element modeling impractical, so the models used represent planar sections of blocks of fiber composites in a thermal conductivity apparatus. Thermal parameters of a material are assumed to be invariant.

Wu, F., 1988, A 3d model of a joint rock mass and its deformation properties, International Journal of Rock Mechanics and Mining Sciences \& Geomechanics Abstracts, v. 6, no. 2, p. 169-177.

\section{Appendix.6: Volumetric Averaging Techniques}

Cai, M. and H. Horii, 1993, A constitutive model and FEM analysis of jointed rock masses, International Journal of Rock Mechanics and Mining Sciences \& Geomechanics Abstracts, v. 30, no. 4, p. 351-359.

A constitutive model of jointed rock masses is presented which reflects the size, density, orientation, and connectivity of joints as well as their mechanical properties. Following the continuum approach, the incremental stress-strain relation of the jointed rock mass is formulated by taking the volume average of stress and strain inside a representative volume element where the evaluation of the relative displacement across the joints is required. Employing an elasto-plastic constitutive model of the joint behavior, the relative displacement across the joint can be obtained once the stress acting on the joint is known. The fundamental difficulty in the constitutive modeling of jointed rock masses is due to the fact that the stress acting on a joint is different from the avg. stress since a joint does not cut through the rock mass but terminates within the rock mass, possible connecting w/ other joints. The lower the stiffness of the surrounding matrix is, due to the existence of other joints of the connection of joints, the higher the stress acting on 
the joint will be. In the present study, the stress concentration tensor, which gives a relation between avg. stress and the stress acting on the joint, is introduced and a simple method to evaluate it is developed. The interaction effect between joints and the effect of joint connection are properly considered in the model. Some simple examples are solved by the proposed constitutive model. The results are in agreement with experimental data showing the characteristic features of the behavior of jointed rock masses. The proposed constitutive model for jointed rock masses is implemented into a finite element analysis program with a 3-D isoparametric element to analyze actual engineering problems. As an example, the program is used to analyze a plate-loading test problem and the results of the 3-D finite element analysis of the problem are compared w/ the test data. For highly jointed rock masses, the continuum model offers a powerful analytical method.

Grossmann, N.F., 1983, A numerical method for the definition of discontinuity sets, Fifth International Congress on Rock Mechanics, Melbourne, Australia, Boston, MA: A.A. Balkema, p. B17-B21.

This paper describes the numerical method developed at the LNEC which allows the complete determination of the existing discontinuity system starting from the total field data collection. The method may be applied to any number of the sets of information obtained and can also take into consideration cases of nonuniform sampling of the joints. Determination of the existing discontinuity sets is done through different steps, the 1st one using as a basis the spherical Poisson distribution and the other ones the bivariate normal distribution on the tangent plane at the mean attitude.

Heuze, F.E., 1980, Scale effects in the determination of rock mass strength and deformability, Rock Mechanics, v. 12, p. 167-192.

An extensive literature search was conducted, to investigate the effect of the scale of testing on the measurement of strength and stiffness of rock masses. The results are presented for bearing capacity tests, compression tests, and several types of deformability tests. Field strength values are generally several times smaller than laboratory values. Field moduli generally appear to be between $20 \%$ and $60 \%$ of lab measured moduli. All strength and deformability results reviewed point to the great need for more field tests. Specific suggestions are given.

Kovari, K., 1982, Rock mass behaviour and its mathematical modeling, Numerical Methods in Geomechanics, Proceedings of the NATO Advanced Study Institute, August 24September 4, 1981, University of Minho, Vimeiro, Portugal, Hingham, MA: Kluwer Boston, p. 145-163.

The behavior of rock masses is largely determined by: orientation and nature of its discontinuities and properties of the intact rock. Deformation and failure may take place in both parts resulting in the actual response of the rock mass to changes 
in stresses. Rock masses may behave both in a brittle or a ductile manner depending on the state of stress they are subjected to. It is of fundamental significance to understand the mechanism of deformation and failure as a process. For this purpose experimental evidence gained from laboratory testing will be discussed. The mathematical modeling of the observed phenomena will be done taking into consideration elasticity, internal friction, cracking, and viscosity. Special attention is given to the problems of the continuum mechanics approach in tunneling practice.

Lundquist, R., J. Fraser, and D. Ross-Brown, 1989, A Bayesian analysis method for incorporating expert opinion and off-site data into rock mechanics studies, Rock Mechanics as a Guide for Efficient Utilization of Natural Resources, Proceedings of the 30th U.S. Symposium, June 19-22, 1989, Morgantown, WV, A.W. Khair (ed.), Brookfield, VT: A.A. Balkema, p. 219-226.

Maier, G. and G. Gioda, 1982, Optimization methods for parametric identification of geotechnical systems, Numerical Methods in Geomechanics, Proceedings of the NATO Advanced Study Institute, August 24-September 4, 1981, University of Minho, Vimeiro, Portugal, Hingham, MA: Kluwer Boston, p. 273-304.

Parameters which characterize geotechnical systems and are representative of the behavior of soil or rock masses are often known with a high degree of uncertainty. A way of reducing uncertainties \& thus improving mathematical models for analysis and design purposes is provided by a systematic adjustment of parameters so that theoretical predictions by the model match observational data. This identification or "inverse" problem frequently implies recourse to techniques of mathematical optimization and particularly of mathematical programming. this paper concerns the role played in this context by direct search methods, least squares procedures, and quadratic and nonlinear programming for identifying parameters in purely numerical models, linear models, and linear complementarity models, respectively. A procedure of statistical identification is briefly mentioned at the end. The scope is not to survey the field but merely to elucidate some aspects and potentialities of identification methods in geomechanics on the basis of a variety of recent results on particular problems.

Nguyen, V.U. and E. Ashworth, 1985, Rock mass classification by fuzzy sets, Research and Engineering Applications in Rock Masses, 26th U.S. Symposium on Rock Mechanics, June 26-28, 1985, Rapid City, SD, E. Ashworth (ed.), Boston, MA: A.A. Balkema, p. 937-945.

This paper presents application of fuzzy set theory (especially the BellmanZadeh aggregation procedure) for obtaining a rock mass classification rating from the CSIR system with incorporation of expert knowledge. Numerical examples are given. 
Ohnishi, Y., T. Mimuro, N. Takewaki, and I. Yoshida, 1985, Verification of input parameters for distinct element analysis of jointed rock mass, Proceedings of the International Symposium on Fundamentals of Rock Joints, September 15-20, 1985, Bjorkliden, Sweden, O. Stephansson (ed.), Lulea, Sweden: CENTEK Publ., p. 205-214.

This paper describes how to evaluate the five input parameters for the Distinct Element Method in analyzing the behavior of a jointed rock mass.

Pariseau, W.G., 1993, Equivalent properties of jointed Biot material, International Journal of Rock Mechanics and Mining Sciences \& Geomechanics Abstracts, v. 30, no. 7, p. 1151-1157.

In many practical cases, rock mass structural and material discontinuities are too numerous to be accounted for on a one-to-one basis, so that an equivalent properties approach is necessary. All existing equivalent properties formulations are based on the assumption of an REV that severely restricts application to engineering design. Application of a non-REV procedure to coupled, poroelastic constitutive equations is described here. The results are in excellent agreement with constraints intrinsic to Biot's material law and with previous non-REV results obtained in application to Hooke's law in linear elasticity and to Darcy's law in seepage analysis. Results for the classic 1-D consolidation problem of a layered isotropic material are in reasonable agreement $\mathrm{w} /$ results for the same sample characterized by equivalent homogeneous, anisotropic properties.

Peres-Rodrigues, F., 1983, Simultaneous influence of several parameters over rock and rock mass properties, Fifth International Congress on Rock Mechanics, Melbourne, Australia, Boston, MA: A.A. Balkema, p. A37-A41.

This is a contribution to the knowledge of the joint influence of several parameters such as anisotropy, heterogeneity, scale effect, time, and temperature over the properties of rocks and rock masses. This influence will be dealt with following a statistical approach. General expressions will be presented to reproduce such an influence, which as a rule are sets of families of anisotropy surfaces. Lastly an instance of application to the study of the deformability is presented, taking into account the simultaneous influence of anisotropy and scale effect.

Pine, R.J., A. Jupe, and L.W. Tunbridge, 1990, An evaluation of in-situ stress measurements affecting different volumes of rock in the Carnmenellis granite, Scale Effects in Rock Masses, Proceedings of the First International Workshop, June 7-8, 1990, Loen, Norway, A. Pinto Da Cunha (ed.), Brookfield, VT: A.A. Balkema, p. 269278. 


\section{Appendix.7: General Relationships}

Bauer, S.J., R.K. Thomas, and L.M. Ford, 1985, Measurement and calculation of the mechanical response of a highly fractured rock, Research and Engineering Applications in Rock Masses, 26th U.S. Symposium on Rock Mechanics, June 26-28, 1985, Rapid City, SD, E. Ashworth (ed.), Boston, MA: A.A. Balkema, p. 523-530.

We have completed the first steps in attempting to validate a material constitutive model for a jointed rock mass. The continuum model, as utilized here within a finite element code, consists of a material constitutive description based on. the linear elastic behavior of the matrix material and nonlinear normal and shear behavior of fracture planes. The validation exercise consists of (1) characterizing an appropriate physical model, (2) measuring the mechanical response of that physical model, and (3) using the physical model characteristics to calculate the mechanical response of the physical model. Calculated and measured responses for small-scale physical models (thermally-fractured granite) are in qualitative agreement. For deviatoric loadings, the calculated tangent modulus is an order of magnitude less than that of the matrix and exhibits stiffening with increasing load, and thereby functionally tracks the measured response

Buckley, J.T., Y.C. Kim, M.S. Nataraja, and D.H. Tiktinsky, 1986, Evaluating host rock representativeness through geostatistics, Rock Mechanics: Key to Energy Production, Proceedings of the 27th U.S. Symposium, June 23-25, 1986, Tuscaloosa, AL, H.L. Hartman (ed.), Brookfield, VT: A.A. Balkema, p. 821-826.

Site characterization includes an exploration and research program in the field and laboratory to determine the geologic conditions and ranges of those parameters which are necessary for determining the suitability of the site as a geologic repository. The information thus collected should be able to establish with reasonable assurance that the public and the environment will be adequately protected from the hazards associated w/ a repository. This paper demonstrates by example a geostatistical approach which, on the basis of the existing limited database, can be used to evaluate (1) whether existing information on a certain parameter is adequate to be considered representative, (2) if additional information is needed, where should additional sampling/testing be performed, and (3) whether the newly acquired data along $\mathrm{w} /$ the existing information constitute representative data.

Heuze, F.E., 1980, Scale effects in the determination of rock mass strength and deformability, Rock Mechanics, v. 12, p. 167-192.

An extensive literature search was conducted, to investigate the effect of the scale of testing on the measurement of strength and stiffness of rock masses. The results are presented for bearing capacity tests, compression tests, and several types 
of deformability tests. Field strength values are generally several times smaller than laboratory values. Field moduli generally appear to be between $20 \%$ and $60 \%$ of lab measured moduli. All strength and deformability results reviewed point to the great need for more field tests. Specific suggestions are given.

Nguyen, V.U. and E. Ashworth, 1985, Rock mass classification by fuzzy sets, Research and Engineering Applications in Rock Masses, 26th U.S. Symposium on Rock Mechanics, June 26-28, 1985, Rapid City, SD, E. Ashworth (ed.), Boston, MA: A.A. Balkema, p. 937-945.

This paper presents application of fuzzy set theory (especially. the BellmanZadeh aggregation procedure) for obtaining a rock mass classification rating from the CSIR system with incorporation of expert knowledge. Numerical examples are given.

Pariseau, W.G., 1993, Equivalent properties of jointed Biot material, International Journal of Rock Mechanics and Mining Sciences \& Geomechanics Abstracts, v. 30, no. 7, p. 1151-1157.

In many practical cases, rock mass structural and material discontinuities are too numerous to be accounted for on a one-to-one basis, so that an equivalent properties approach is necessary. All existing equivalent properties formulations are based on the assumption of an REV that severely restricts application to engineering design. Application of a non-REV procedure to coupled, poroelastic constitutive equations is described here. The results are in excellent agreement with constraints intrinsic to Biot's material law and with previous non-REV results obtained in application to Hooke's law in linear elasticity and to Darcy's law in seepage analysis. Results for the classic 1-D consolidation problem of a layered isotropic material are in reasonable agreement $\mathrm{w} /$ results for the same sample characterized by equivalent homogeneous, anisotropic properties.

Shurtz, R.F., 1992, Pseudo-fractal interpolation for risk analysis, Mathematical Geology, v. 24, no. 1, p. 99-128.

This paper describes a new method of analyzing the risk incurred when the outcome of a decision depends on interpolated values, for example, on the flow through an aquifer sparsely sampled for permeability or on the ratio of waste to ore in a mineral deposit sparsely sampled for grade. The method uses large families of interpolations constructed between sample values using adaptations of the wellknown midpoint displacement method for generating pseudo-fractional Brownian motion trajectories. The parameters defining each family are chosen interactively by specialists to incorporate their expert knowledge. Each family, or ensemble, then defines a population of values for any global characteristic (functional) such as flowrate or waste ratio. The probabilities of various outcomes are estimated by 
counting them and calculating their ratios. For example, if 900 out of 1000 are acceptable the chance of success is estimated to be $90 \%$.

Yegulalp, T.M. and K. .Kim, 1992, Statistical assessment of scale effect on rock properties using the theory of extremes, AIME Preprint 92-30, Littleton. CO: Society of Mining Engineers of AIME (available from Linda Hall Library, Kansas City, MO, 1-800662-1545.)

A new explanation of the scale dependency of the strength properties of rock is presented based on the theory of extremes. A review of past work on specimen size strength relationship is followed by an introduction to the fundamentals of the underlying theory of extremes and its relationship to the scale effect on the statistical properties of the strength distribution. It is shown that the weakest link theory is directly related to external statistics and properties of strength distributions of rock can be derived as a function of the specimen size. Three sets of Brazilian test data are used to demonstrate the use of this approach

\section{Appendix.8: Case Studies}

Jackson, R. and J.S.O. Lau, 1990, The effect of specimen size on the laboratory mechanical properties of Lac du Bonnet grey granite, Scale Effects in Rock Masses, Proceedings of the First International Workshop, June 7-8, 1990, Loen, Norway, A. Pinto Da Cunha (ed.), Brookfield, VT: A.A. Balkema, p. 165-174.

Long, J.C.S., K. Karasaki, A. Davey, J. Peterson, M. Landsfeld, J. Kemeny, and S. Martel, 1991, An inverse approach to the construction of fracture hydrology models conditioned by geophysical data: an example from the validation exercises at the Stripa Mine, International Journal of Rock Mechanics and Mining Sciences \& Geomechanics Abstracts, v. 28, no. 2/3, p. 121-142.

Munier, R., 1993, Four-dimensional analysis of fracture arrays at the Aspo Hard Rock Laboratory, SE Sweden, Engineering Geology, v. 33, no. 3, p. 159-175.

During excavation and construction of Aspo HRL, geological info is systematically recorded and filed in a database called GEOTAB. Statistical analyses have been performed on fracture info from GEOTAB and stochastically chosen outcrops have been mapped in detail. Based on systematic recording of crosscutting relations, a succession of minerals and characteristics of decreasing age have been outlined. These include epidote, quartz, oxidized walls, iron oxides, chlorite, and calcite. The sequential fragmentation of the rock mass is described in terms of evolution of fracture arrays in time. A correction procedure for sampling biases has been performed and fracture traits have been analyzed. It is concluded that fracture lengths follow a lognormal distribution w/ mean, mode, and std dev 
estimated. Spacing has been evaluated for different fracture sets. It is concluded that the fracture array consists of six sets with different kinematics. Fracture zones underground are compared to a fracture zones at the surface.

Pine, R.J., A. Jupe, and L.W. Tunbridge, 1990, An evaluation of in-situ stress measurements affecting different volumes of rock in the Carnmenellis granite, Scale Effects in Rock Masses, Proceedings of the First International Workshop, June 7-8, 1990, Loen, Norway, A. Pinto Da Cunha (ed.), Brookfield, VT: A.A. Balkema, p. 269278.

Pinnaduwa, H.S.W., N.W. Deepa, and O. Stephansson, 1990, Three dimensional stochastic joint geometry modeling including a verification; a case study, Rock Mechanics: Contributions and Challenges, Proceedings of the 31st U.S. Symposium, June 1820, 1990, Golden, CO, W.A. Hustrulid and G.A. Johnson (eds.), Brookfield, VT: A.A. Balkema, p. 1051-1058.

Since joint geometry patterns can vary from one region to another, each region should be represented by a separate joint geometry model. Therefore, the first step should be identification of statistically homogeneous regions. It then is necessary to know the number of joint sets, and for each joint set, the intensity, spacing, location, orientation, shape, and dimension distributions. These joint parameters are inherently statistical. Sample values of joint parameters provided by the field data usually are subject to sampling biases and represent only 1- or 2-D properties. Therefore, before inferring these parameters from sampling values, field data should be corrected for sampling biases. In addition, principles of stereology must be used to infer 3D parameters from 1- or 2-D values. At present, a 3-D joint geometry modeling scheme which incorporates these features and also includes a formal verification procedure is not available in the literature. This paper shows the development of 3-D joint geometry models which investigate statistical homogeneity, include correction for sampling biases, and applications of stereological principles. It also checks the validity of such models (?).

Pinto Da Cunha, A. and J. Muralha, 1990, About LNEC experience on scale effects in the deformability of rock masses, Scale Effects in Rock Masses, Proceedings of the First International Workshop, June 7-8, 1990, Loen, Norway, A. Pinto Da Cunha (ed.), Brookfield, VT: A.A. Balkema, p. 219-229.

A review of several deformability studies of rock masses carried out by LNEC, $\mathrm{w} / \mathrm{a}$ reanalysis and a reinterpretation of existent data is presented, emphasizing the variation of the rock mass deformability moduli w/ the tested volumes. Since different laboratory and in situ tests were considered for each site, an attempt to understand the associated scale effects is made, taking into due consideration the influence of distinct testing techniques and potential sampling bias in the variation and scattering of the test results. 
Sage, J.D., A.A. Aziz, and E.R. Danek, 1990; Aspects of scale effects on rock closure, Scale Effects in Rock Masses, Proceedings of the First International Workshop, June 7-8, 1990, Loen, Norway, A. Pinto Da Cunha (ed.), Brookfield, VT: A.A. Balkema, p. 175-181.

Difficulties in predicting the load-deformation behavior of jointed rock which is based on lab or field studies on small samples are first described. Theoretical studies of rock opening and closure based on an analysis of joint surface topography are presented which suggest a scaling factor based on sample size and deformation history of the insitu jointed rock mass. The data from lab loaddeformation studies of rock closure on models of jointed rock specimens ranging in size from $100-900 \mathrm{~cm}^{2}$ coupled $\mathrm{w} /$ the results of profilometer tests on the joint surfaces and finite element analysis is presented in support of theoretical considerations.

Zimmerman, R.M. and M.L. Blanford, 1985, Evaluation of the accuracy of continuum-based computational models in relation to field measurements in welded tuff, Proceedings of the International Symposium on Fundamentals of Rock Joints, September 15-20, 1985, Bjorkliden, Sweden, O. Stephansson (ed.), Lulea, Sweden: CENTEK Publ., p. 233-245.

\section{Appendix.9: Miscellaneous}

Fattohi, Z.R. and B. Al-kayat, 1993, Interpretation of reservoir rock mechanical properties by direct methods; a study on two oil-bearing limestone units, Environmental Geology, v. 21 , no. $1-2$, p. $37-41$.

Direct core analysis results of bulk density, porosity, permeability, resistivity, transit time, and strength were correlated w/ logging data from an oil well. The interpretations were made in terms of index values between field and lab data for the 2 units studied. An engineering classification scheme was suggested. Other rock engineering properties such as sonic velocity and dynamic modulus were calculated as well. An attempt was made to predict the fracture pressure gradient of the rock formation. This work has revealed the possibility of using directly measured data to evaluate some of the in situ rock behavior.

Fukushima, K., 1990, Scale effects on underground excavations, Scale Effects in Rock Masses, Proceedings of the First International Workshop, June 7-8, 1990, Loen, Norway, A. Pinto Da Cunha (ed.), Brookfield, VT: A.A. Balkema, p. 209-217. 
Ito, T., K. Hayaski, and H. Abe, 1990, Scale effect in breakdown pressure of hydraulic fracturing stress measurements, Scale Effects in Rock Masses, Proceedings of the First International Workshop, June 7-8, 1990, Loen, Norway, A. Pinto Da Cunha (ed.), Brookfield, VT: A.A. Balkema, p. 289-297.

The mechanism of crack initiation from a wellbore due to pressurization was studied theoretically and experimentally to understand the scale effect existing in the breakdown pressure of hydraulic fracturing stress measurements. Laboratory hydraulic fracturing was conducting in two kinds of rock. The resulting breakdown pressure clearly tended to decrease w/ increasing wellbore diameter. This scale effect is successfully explained by a new criterion that assumed that cracks initiate when the maximum principal stress reaches the tensile strength at a point at a distance from the stress concentration point. This distance is a material constant (called the characteristics length of tensile failure of the rock). Here, the char. length was evaluated from the tensile strength and fracture toughness from 3-point bending. The conventional approach, which determines the breakdown pressure as a pressure valued at which the maximum hoop stress on the wellbore surface reaches the tensile strength, results in breakdown pressure that is independent of the wellbore diameter, and thus cannot explain this scale effect.

Pinto Da Cunha, A., (ed.), 1990, Scale Effects in Rock Masses, Proceedings of the First International Workshop, June 7-8, 1990, Loen, Norway, Brookfield, VT: A.A. Balkema.

Price, R.H. and S.J. Bauer, 1985, Analysis of the elastic and strength properties of Yucca Mountain tuff, Nevada, Research and Engineering Applications in Rock Masses, 26th U.S. Symposium on Rock Mechanics, June 26-28, 1985, Rapid City, SD, E. Ashworth (ed.), Boston, MA: A.A. Balkema, p. 89-96.

Determines empirical relationships of Young's modulus vs. porosity and ultimate stress vs. porosity for samples of Yucca Mtn. silicic tuffs, extended to include the montmorillonite volume fraction as a "functional porosity".

Zimmerman, R.M. and M.L. Blanford, 1985, Evaluation of the accuracy of continuum-based computational models in relation to field measurements in welded tuff, Proceedings of the International Symposium on Fundamentals of Rock Joints, September 15-20, 1985, Bjorkliden, Sweden, O. Stephansson (ed.), Lulea, Sweden: CENTEK Publ., p. 233-245. 


\section{YUCCA MOUNTAIN SITE CHARACTERIZATION PROJECT \\ SAND95-2338 - DISTRIBUTION LIST \\ $8 / 15 / 96$}

1

D. A. Dreyfus (RW-1)

Director

OCRWM

US Department of Energy

1000 Independence Avenue SW

Washington, DC 20585

L. H. Barrett (RW-2)

Acting Deputy Director

OCRWM

US Department of Energy

1000 Independence Avenue SW

Washington, DC 20585

1 S. Rousso (RW-40)

Office of Storage and Transportation

OCRWM

US Department of Energy

1000 Independence Avenue SW

Washington, DC 20585

1

R. A. Milner (RW-30)

Office of Program Management

and Integration

OCRWM

US Department of Energy

1000 Independence Avenue SW

Washington, DC 20585

1 D. R. Elle, Director

Environmental Protection Division

DOE Nevada Field Office

US Department of Energy

P.O. Box 98518

Las Vegas, NV 89193-8518

1 T. Wood (RW-14)

Contract Management Division

OCRWM

US Department of Energy

1000 Independence Avenue SW

Washington, DC 20585

$4 \quad$ Victoria F. Reich, Librarian

Nuclear Waste Technical Review Board

1100 Wilson Blvd., Suite 910

Arlington, VA 22209

1 Wesley Barnes, Project Manager

Yucca Mountain Site Characterization

Office

US Department of Energy

P.O. Box 98608-MS 523

Las Vegas, NV 89193-8608
1 Director, Public Affairs Office

c/o Technical Information Resource Center

DOE Nevada Operations Office

US Department of Energy

P.O. Box 98518

Las Vegas, NV 89193-8518

Technical Information Officer

DOE Nevada Operations Office

US Department of Energy

P.O. Box 98518

Las Vegas, NV 89193-8518

J. R. Dyer, Deputy Project Manager

Yucca Mountain Site Characterization Office US Department of Energy

P.O. Box 98608 - MS 523

Las Vegas, NV 89193-88608

1

M. C. Brady

Laboratory Lead for YMP

M\&O/Sandia National Laboratories

1261 Town Center Drive

Bldg. 4, Room 421A

Las Vegas, NV 89134

J. A. Canepa

Laboratory Lead for YMP

EES-13, Mail Stop J521

M\&O/Los Alamos National Laboratory

P.O. Box 1663

Los Alamos, NM 87545

Repository Licensing \& Quality

Assurance

Project Directorate

Division of Waste Management, MS T7J-9

US NRC

Washington, DC 20555

Senior Project Manager for Yucca

Mountain

Repository Project Branch

Division of Waste Management, MS T7J-9

US NRC

Washington, DC 20555

$1 \quad$ NRC Document Control Desk

Division of Waste Management, MS T7J-9

US NRC

Washington, DC 20555 
NRC Site Representative

301 E Stewart Avenue, Room 203

Las Vegas, NV 89101

1

Center for Nuclear Waste

Regulatory Analyses

Southwest Research Institute

6220 Culebra Road

Drawer 28510

San Antonio, TX 78284

2

W. L. Clarke

Laboratory Lead for YMP

M\&O/ Lawrence Livermore Nat'1 Lab

P.O. Box 808 (L-51)

Livermore, CA 94550

$1 \quad$ Robert W. Craig

Acting Technical Project Officer/YMP

US Geological Survey

101 Convention Center Drive, Suite P-110

Las Vegas, NV 89109

1 J. S. Stuckless, Chief

Geologic Studies Program

MS 425

Yucca Mountain Project Branch

US Geological Survey

P.O. Box 25046

Denver, CO 80225

$1 \quad$ L. D. Foust

Technical Project Officer for YMP

TRW Environmental Safety Systems

101 Convention Center Drive

Suite P-110

Las Vegas, NV 89109

1

A. L. Flint

U. S. Geological Survey

MS 721

P. O. Box 327

Mercury, NV 89023

$1 \quad$ Robert L. Strickler

Vice President \& General Manager

TRW Environmental Safety Systems, Inc.

2650 Park Tower Dr.

Vienna, VA 22180

1 Jim Krulik, Geology Manager

US Bureau of Reclamation

Code D-8322

P.O. Box 25007

Denver, CO 80225-0007
B. T. Brady

Records Specialist

US Geological Survey

MS 421

P.O. Box 25046

Denver, CO 80225

1

2

M. D. Voegele

Deputy of Technical Operations

M\&O/SAIC

101 Convention Center Drive

Suite P-110

Las Vegas, NV 89109

\section{A. T. Tamura}

Science and Technology Division

OSTI

US Department of Energy

P.O. Box 62

Oak Ridge, TN 37831

1

1

P. J. Weeden, Acting Director

Nuclear Radiation Assessment Div.

\section{US EPA}

Environmental Monitoring Sys. Lab P.O. Box 93478

Las Vegas, NV 89193-3478

John Fordham, Deputy Director

Water Resources Center

Desert Research Institute

P.O. Box 60220

Reno, NV 89506

1

The Honorable Jim Regan

Chairman

Churchill County Board of

Commissioners

$10 \mathrm{~W}$. Williams Avenue

Fallon, NV 89406

R. R. Loux

Executive Director

Agency for Nuclear Projects

State of Nevada

Evergreen Center, Suite 252

1802 N. Carson Street

Carson City, NV 89710

Brad R. Mettam

Inyo County Yucca Mountain

Repository Assessment Office

P. O. Drawer L

Independence, CA 93526

1 Vernon E. Poe

Office of Nuclear Projects

Mineral County

P.O. Box 1600

Hawthorne, NV 89415 
Les W. Bradshaw

Program Manager

Nye County Nuclear Waste Repository

Project Office

P.O. Box 1767

Tonopah, NV 89049

$1 \quad$ Florindo Mariani

White Pine County Coordinator

P. O. Box 135

Ely, NV 89301

1 Tammy Manzini

Lander County Yucca Mountain Information Officer

P.O. Box 10

Austin, NV 89310

1 Jason Pitts

Lincoln County Nuclear Waste Program Manager

P. O. Box 158

Pioche, NV 89043

1 Dennis Bechtel, Coordinator

Nuclear Waste Division

Clark County Dept. of Comprehensive Planning

P.O. Box 55171

Las Vegas, NV 89155-1751

1 Juanita D. Hoffman

Nuclear Waste Repository

Oversight Program

Esmeralda County

P.O. Box 490

Goldfield, NV 89013

1 Sandy Green

Yucca Mountain Information Office

Eureka County

P.O. Box 714

Eureka, NV 89316

1 Economic Development Dept.

City of Las Vegas

400 E. Stewart Avenue

Las Vegas, NV 89101

1 Community Planning \& Development

City of North Las Vegas

P.O. Box 4086

North Las Vegas, NV 89030

2 Librarian

YMP Research \& Study Center

101 Convention Center Drive, Suite P-110

Las Vegas, NV 89109
1

Library Acquisitions

Argonne National Laboratory

Building 203, Room CE-111

9700 S. Cass Avenue

Argonne, IL 60439

1

Glenn Van Roekel

Manager, City of Caliente

P.O. Box 158

Caliente, NV 89008

G. S. Bodvarsson

Head, Nuclear Waste Department

Lawrence Berkeley National Laboratory

1 Cyclotron Road, MS 50E

Berkeley, CA 94720

1

Steve Hanauer (RW-2)

OCRWM

U. S. Department of Energy

1000 Independence Ave.

Washington, DC 20585

MS

$1 \quad 1324$

$10 \quad 1324$

1324

1326

1326

1330

1330

C. Ho, 6115

S. McKenna, 6115

C. Rautman, 6115

B. Amold 6851

S. Altman, 6851

B. Pierson, 6811

100/SAND95-2338/NQ

20

WMT Library, 6752

9018

Central Technical Files, 8523-2

0899 Technical Library, 4414

0619 Review and Approval Desk, 12630, For DOE/OSTI

1 Dr. Eileen Poeter

Dept. of Geology and Geological Eng.

Colorado School of Mines

Golden, CO 80401

1 Dr. Kadri Dagdelen

Dept. Of Mining Engineering

Colorado School of Mines

Golden, CO 80401

1 Dr. Andrew Wolfsberg

Los Alamos National Laboratory

Dept. EES-5

MS F665

Los Alamos, NM 87545 
1 Dr. Alan Flint U. S. Geological Survey

Bank of America Center, Suite 860

101 Convention Center Drive

Las Vegas, NV 89109

1 Mr. Jeff Brown

Dept. of Geology and Geological Eng.

Colorado School of Mines

Golden;'CO 80401

1 Dr. Ross Bagtzoglou

CNWRA

6220 Culebra Road

San Antonio, TX 78238-5166

1 Dr. Larry Anna

U. S. Geological Survey

Box 25046, MS 421

Lakewood, CO 80225

1 Dr. Mark Bandurraga Lawrence Berkeley Laboratory 1 Cyclotron Road, MS 50E

Berkeley, CA 94720

1 Dr. George Zyvolovski

Los Alamos Natioal Laboratory

Dept. EES-5

MS J521

Los Alamos, NM 87545

1 Dr. Srkanta Mishra

$\mathrm{M} \& O / I n t e r a$ Inc.

101 Convention Center Drive

Suite P110

Las Vegas, NV 